\title{
A Framework for Geometric Field Theories and their Classification in Dimension One
}

\author{
Matthias LUDEWIG ${ }^{\mathrm{a}}$ and Augusto STOFFEL ${ }^{\mathrm{b}}$ \\ a) Universität Regensburg, Germany \\ E-mail: matthias.ludewig@mathematik.uni-regensburg.de \\ b) Universität Greifswald, Germany \\ E-mail: arstoffel@gmail.com
}

Received June 15, 2020, in final form July 12, 2021; Published online July 25, 2021

https://doi.org/10.3842/SIGMA.2021.072

\begin{abstract}
In this paper, we develop a general framework of geometric functorial field theories, meaning that all bordisms in question are endowed with geometric structures. We take particular care to establish a notion of smooth variation of such geometric structures, so that it makes sense to require the output of our field theory to depend smoothly on the input. We then test our framework on the case of 1-dimensional field theories (with or without orientation) over a manifold $M$. Here the expectation is that such a field theory is equivalent to the data of a vector bundle over $M$ with connection and, in the nonoriented case, the additional data of a nondegenerate bilinear pairing; we prove that this is indeed the case in our framework.
\end{abstract}

Key words: field theory; vector bundles; bordism

2020 Mathematics Subject Classification: 57R56; 14D21; 57R22

\section{Introduction}

Inspired by work of Witten [16], Segal and Atiyah pioneered the mathematical description of quantum field theories as functors $[1,12]$. More precisely, they described a $d$-dimensional quantum field theory $Z$ as a functor that assigns to a closed $(d-1)$-manifold $Y$ a vector space $Z(Y)$ and to a $d$-dimensional bordism $X$ from $Y$ to another closed $d$-manifold $Y^{\prime}$ a linear map $Z(X): Z(Y) \rightarrow Z\left(Y^{\prime}\right)$. Moreover, $Z$ is required to be a symmetric monoidal functor, which means that $Z$ applied to a disjoint union of manifolds of dimension $d-1$ or $d$ corresponds to the tensor product of the associated vector spaces or linear maps. Segal's paper focused on conformal field theories, which means that the manifolds involved come equipped with conformal structures, while Atiyah discusses topological field theories, where the manifolds are smooth, but not equipped with any additional geometric structure.

Our first goal in this paper is to develop a general framework for geometric field theories. This involves a general definition of a "geometric structure" $\mathcal{G}$ on $d$-dimensional manifolds, which then leads to the definition of a symmetric monoidal bordism category $\mathcal{G}$ Bord whose morphisms are $d$-dimensional bordisms equipped with a $\mathcal{G}$-structure. This is much more general than the conformal structures considered by Segal or the rigid structures based on the action of a Lie group $G$ on a $d$-dimensional model space $\mathbb{M}$ of Stolz and Teichner [13]. Then we essentially follow [13] to define $\mathcal{G}$-field theories. As discussed at length in that paper, it is crucial to ensure the smoothness of the field theories; intuitively, this means in particular that the operator $Z(X): Z(Y) \rightarrow Z\left(Y^{\prime}\right)$ associated to a bordism $X$ from $Y$ to $Y^{\prime}$ depends smoothly on the bordism $X$. At a technical level, this means that we need "family versions" of the bordism category $\mathcal{G}$ Bord and the target category Vect of suitable vector spaces whose objects and morphisms are now families of the originally considered objects/morphisms, parametrized by 
smooth manifolds. In [13] this is implemented by considering $\mathcal{G}$ Bord and Vect as categories internal to the 2-category of smooth stacks, but it has become clear that, for technical reasons, it is easier to construct and to work with the complete Segal object in smooth stacks that should be thought of as the "nerve" of the internal category we considered before, as is done in the preprint [2]. We carry out our constructions for non-extended field theories. It is possible to define extended field theories using an extension of our approach, via $d$-fold (or $d$-uple) Segal objects. For one-dimensional field theories, which are the main object of study in this article, these distinctions are irrelevant.

The second goal of this paper is to check whether this abstract and involved definition yields something sensible in the simplest cases, namely 1-dimensional (oriented) topological field theories over a manifold $M$. In other words, the geometric structure on 1-manifolds $X$ is simply a smooth map $\gamma: X \rightarrow M$, or such a map $\gamma$ plus an orientation on $X$.

Theorem 1.1. The groupoid of 1-dimensional oriented topological field theories over $M$ is equivalent to the groupoid of finite-dimensional vector bundles with connections over $M$.

Theorem 1.2. The groupoid of 1-dimensional unoriented topological field theories over $M$ is equivalent to the groupoid of finite-dimensional vector bundles over $M$ equipped with a nondegenerate, possibly indefinite, field of symmetric bilinear forms on the fibers and a compatible connection.

There are actually two versions of each of these results, depending on whether all vector spaces involved are real or complex. For the field theories, the two flavors come from the choice of the target category as the category (of families of) real or complex vector spaces. Similarly, the vector bundles over $M$ considered can be real or complex.

Theorem 1.1 is certainly the expected result. The basic idea is that a vector bundle $E \rightarrow M$ with connection $\nabla$ determines a 1-dimensional field theory $Z$ over $M$ which associates to a point $x$ (interpreted as an object of $\mathcal{G}$ Bord) the vector space $Z(x)=E_{x}$ given by the fiber over $x$, and to a path $\gamma:[a, b] \rightarrow M$ (interpreted as a morphism in $\mathcal{G}$ Bord from $\gamma(a)$ to $\gamma(b)$ ) the linear map $Z(\gamma): Z(x) \rightarrow Z(y)$ given by parallel translation along the path $\gamma$.

In fact, there are closely related results in the literature, in work of Freed [4, Appendix A], and, in particular, in the papers by Schreiber and Waldorf [10] and by Berwick-Evans and Pavlov [2], whose title indeed seems a statement of our first theorem. Indeed, our framework is closely related to that of the latter paper; however, our goal to give a general definition of geometric bordism categories leads to a different bordism category even in dimension one, as explained below (see Section 2.1). In [3, 10], invariance under "thin" homotopies plays a prominent role. These concepts turned out to be not relevant to the present paper, as we were able to prove the main results (in particular Proposition 4.3) without such assumptions.

This paper is organized as follows. In Section 2, we give a more detailed exposition of our construction and discuss the differences to the papers cited above. Afterwards, in Section 3, we define our notion of geometry, and use it to define a smooth category of geometric bordisms, for any geometry, in any dimension. Starting in Section 4, we restrict to the case of field theories in dimension one. In particular, we prove a version of the classification Theorems 1.1 and 1.2 under the technical assumption that "families of vector spaces" are finite-dimensional vector bundles. As discussed in Section 2.4 for a geometric field theory $Z$, unlike for topological field theories, the vector space $Z(Y)$ associated to an object $Y$ of the bordism category $\mathcal{G}$ Bord is typically not finite-dimensional. This in turn leads to the requirement that the vector spaces $Z(Y)$ need to be equipped with a topology or a "bornological structure" (see Appendix A) in order to formulate the requirement that the operator $Z(X): Z(Y) \rightarrow Z\left(Y^{\prime}\right)$ associated to a bordism $X$ from $Y$ to $Y^{\prime}$ depends "smoothly" on $X$. As also explained in Section 2.4, the appropriate notion of an " $S$-family of (topological or bornological) vector spaces" needs to be more general 


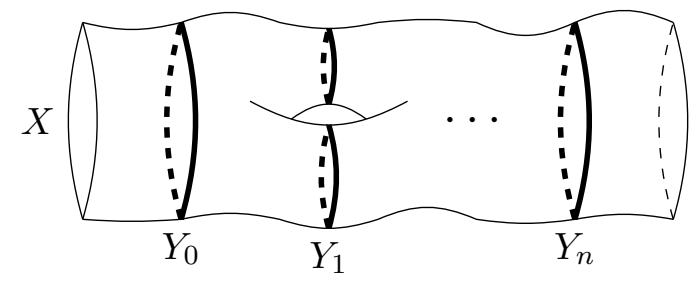

Figure 1. An object of $\mathcal{C}_{n}$ comprises the $d$-manifold $X$ and the marked hypersurfaces $Y_{0}, \ldots, Y_{n}$.

than locally trivial bundles over the parameter spaces $S$, namely sheaves of $\mathcal{O}_{S}$-modules. These are the objects of the target category appropriate for general field theories, and so we consider the version of Theorems 1.1 and 1.2 for that target category as the main result of this paper. This is proved in Section 5 (see Theorem 5.2 and Remark 5.3 for the precise statement).

\section{Discussion of the results}

In this section we provide an informal overview of our framework of geometric field theories, a discussion of our motivations, and comparisons to the existing literature.

\subsection{Bordisms in the Segal approach}

In the presence of geometric structures, it is difficult to perform the gluing of bordisms along their boundaries in a systematic way, as needed to define composition in geometric bordism categories; see for instance the discussion in the introduction of [2]. Here the idea of Segal objects comes to the rescue, as it allows one to instead consider only decomposition of bordisms along hypersurfaces, which is unproblematic. In this approach, a category $C$ is encoded by its nerve, that is, the simplicial set $\mathcal{C}$, where $\mathcal{C}_{0}$ is the set of objects and, for $n \geq 1, \mathcal{C}_{n}$ is the set of chains of $n$ composable morphisms; composition and identity morphisms in $C$ determine the simplicial structure maps between these sets.

To describe, at least roughly, the $d$-dimensional bordism category in this way, we let $\mathcal{C}_{n}$ consist of $d$-dimensional manifolds $X$ together with a collection of compact hypersurfaces $Y_{k} \subset X$, for $k=0, \ldots, n$, as in Figure 1. This encodes a chain of $n$ composable bordisms, the $k$ th of them being the portion of $X$ lying between $Y_{k-1}$ and $Y_{k}$. Composition is encoded by forgetting the marked hypersurfaces. To build in a geometry $\mathcal{G}$ (for instance, orientations, Riemannian metrics, or maps to a background manifold $M$ ), we just ask that $X$ is endowed with that additional structure.

In particular, objects of $\mathcal{C}$ (i.e., elements of $\mathcal{C}_{0}$ ) consist of a $d$-dimensional manifold $X$ with a marked compact hypersurface $Y$, instead of just the $(d-1)$-dimensional manifold $Y$. Now, this set of objects is much larger than what we would like to have, since the portion of $X$ far away from $Y$ should be irrelevant. This issue can be dealt with by promoting $\mathcal{C}_{0}$ from a set to a groupoid; we add isomorphisms that establish suitable identifications between the pairs $Y \subset X$. (The same approach applies later on, as we work fibered over Man, by promoting a certain sheaf to a stack.) This shifts the problem to making a choice of such isomorphisms.

The choice made in [2] is to say that morphisms in $\mathcal{C}_{0}$ are maps $\varphi: Y \rightarrow Y^{\prime}$ that have an extension to a diffeomorphism between open neighborhoods of $Y$ and $Y^{\prime}$ in $X$ and $X^{\prime}$. This makes the concrete embedding of the hypersurface immaterial and ensures that the set of isomorphism classes of objects is precisely the set of $(d-1)$-dimensional manifolds, without any extra data. The issue with this approach is that, while it works in the special case at hand, it does not generalize to arbitrary geometries $\mathcal{G}$, since we are not allowed to restrict a $\mathcal{G}$-structure on $X$ to one on the hypersurface $Y$. Moreover, even for those $\mathcal{G}$ which make sense in any 
dimension and allow restricting to hypersurfaces, it may not be true that a $\mathcal{G}$-isomorphism is determined by its data on a hypersurface.

Our choice for morphisms in $\mathcal{C}_{0}$ is designed to accommodate for any geometry $\mathcal{G}$, in the sense of Section 3.3, and is as follows. First we remark that one of our axioms for a geometry is that one can always restrict it to an open subset of a $\mathcal{G}$-manifold $X$. We then decree that a morphism between two pairs $X \subset Y$ and $X^{\prime} \subset Y^{\prime}$ in $\mathcal{C}_{0}$ is determined by a $\mathcal{G}$-isomorphism defined on an open neighborhood $U \subseteq X$ of $Y$, the underlying smooth map of which sends $Y$ to $Y^{\prime}$; we identify two such $\mathcal{G}$-isomorphisms defined on, say, $U$ and $U^{\prime}$ if they coincide on some smaller neighborhood $V \subset U \cap U^{\prime}$ of $Y$. Concisely, morphisms in $\mathcal{C}_{0}$ are germs of $\mathcal{G}$-isometries at the marked hypersurfaces.

Further stages of the simplicial object $\mathcal{C}$ are constructed in a similar fashion.

\subsection{Points versus germs of paths}

Our definition of $\mathcal{C}_{0}$ raises another difficulty, which is generally unavoidable from our point of view: The set of isomorphism classes of objects is huge, as each different germ of the geometric structure determines its own isomorphism class. This is already true for the case of one-dimensional bordisms over a target manifold $M$, which is considered in this paper. Here, an object in the bordism category can no longer be pictured as a point of $M$ (or, more generally, a finite collection of such); instead, objects are germs of paths in $M$, a much larger space.

The main results of our paper (Theorems 1.1 and 1.2) say that, at least in the one-dimensional case, this does not make a difference: A field theory $Z \in 1$-TFT $(M)$ is completely blind to the germ information, and its value on objects of $\mathcal{C}$ contains no more data than that of a vector bundle over $M$, as expected. This can be seen as a "reality check" for our definition of geometric field theories.

A typical heuristic argument as to why the germs do not matter is that the space of germs of paths in $M$ deformation retracts to $M$. A field theory $Z$ indeed defines a vector bundle on this space of germs, viewed as a diffeological space. However, at this level of generality, the familiar homotopy invariance of vector bundles breaks down. So, instead, we will use the data assigned by $Z$ to higher simplicial levels to show that $\left.Z\right|_{\mathcal{C}_{0}}$ is determined by a vector bundle on $M$.

\subsection{Building in smoothness}

A second technical layer in our framework comes from the need to formalize the idea that our field theories should be smooth. This is already explained in detail in [13] and adapted to the Segal approach in [2]. The idea here is that a smooth category $\mathcal{C}$ is a complete Segal object in the 2-category of (symmetric monoidal) stacks over the site of manifolds; compare this with the preliminary description of above, where $\mathcal{C}$ was a Segal object in the 2-category of (symmetric monoidal) groupoids. Thus, for each integer $n \geq 0$ and each smooth manifold $S$ (a "parameter space"), we have a groupoid $\mathcal{C}_{n, S}$ of $S$-families of chains of $n$ composable morphisms; this data is functorial in the variables $n$ and $S$.

To promote the bordism category to a smooth category, we need to fix the meaning of " $S$ family of bordisms" $X / S$. In a nutshell, this will be defined to be a submersion $\pi: X \rightarrow S$ such that each fiber $\pi^{-1}(s)$ is a bordism in the previous sense. It remains to explain what a geometry $\mathcal{G}$ is in this new context. Before, $\mathcal{G}$ could be defined, technically, to be a sheaf or a stack on the site Man of smooth manifolds; thus, to each $X$, corresponds a set (or groupoid) $\mathcal{G}(X)$ of $\mathcal{G}$ structures on $X$. To extend this to families, we introduce, in Section 3, a new site of families of d-dimensional manifolds, denoted $\mathrm{Fam}^{d}$. Its objects are submersions $\pi: X \rightarrow S$ with $d$ dimensional fibers. A $d$-dimensional geometry is now simply a sheaf or stack on the site Fam ${ }^{d}$. To illustrate this, consider the geometry $\mathcal{G}$ of (fiberwise) Riemannian metrics; if $X / S \in \mathrm{Fam}^{d}$, then $\mathcal{G}(X / S)$ is the set of inner products on the vertical tangent bundle $\operatorname{Ker}(T \pi: T X \rightarrow T S)$. 


\subsection{Appropriate families of vector spaces}

To promote the codomain of our field theories to smooth categories, we must, likewise, specify what we mean by an $S$-family of vector spaces. It is well known that for a topological field theory $Z$ the vector space $Z(Y)$ associated to any object $Y$ of a topological bordism category is finite-dimensional. So it is natural to declare that an $S$-family of vector spaces is simply a finite-dimensional, locally trival smooth vector bundle over $S$, and we will indeed consider exclusively this case in Section 4. Notice that with this choice, a field theory $Z$, as a particular example of a smooth functor, will assign to an $S$-family $X / S$ of bordisms a linear map $Z(X / S)$ of vector bundles over $S$ - an $S$-family of linear maps.

For geometric field theories, the vector spaces $Z(Y)$ are typically not finite-dimensional. For example, the quantum mechanical description of a particle moving in a compact Riemannian manifold $N$ is given by a 1-dimensional Riemannian field theory $Z$ which associates to the object given by $Y=\{0\} \subset(-\epsilon, \epsilon)=X$ (with the standard Riemannian metric on the interval $(-\epsilon, \epsilon)$ ) the "vector space of functions on $N$ ". Let $X / S$ be an $S$-family of bordisms such that for every $s \in S$, the fiber $X_{s}$ is a bordism from $Y$ to $Y^{\prime}$ (where $Y$ and $Y^{\prime}$ do not depend on $s$ ). Then the smoothness requirement for $Z$ in particular says that the maps $S \rightarrow Z\left(Y^{\prime}\right)$ given by $s \mapsto Z\left(X_{s}\right) v$ are smooth, for all $v \in Z(Y)$. If $Z\left(Y^{\prime}\right)$ is infinite dimensional, a topology (or a bornological structure) on $Z\left(Y^{\prime}\right)$ is needed to define a smooth map with target $Z\left(Y^{\prime}\right)$. In quantum mechanics, the vector spaces are traditionally equipped with a Hilbert space structure; for instance, in the case of a particle moving in a Riemannian manifold, the vector space of functions on $N$ is interpreted as $L^{2}(N)$, the Hilbert space of square-integrable functions. However, as discussed in [13, Remark 3.15] there are difficulties formulating the smoothness of the functor $Z$ if the target category is built from families of Hilbert spaces; instead, topological (or bornological) vector spaces are used. In the quantum mechanics example, it is the space $C^{\infty}(N)$ of smooth functions on $N$, equipped with its standard Fréchet topology.

It might seem appealing to define an $S$-family of topological or bornological vector spaces to be a locally trivial bundle of such spaces over $S$ with smooth transition functions. Such a definition, though, has very undesirable consequences for the topology of the space of field theories for a fixed geometry $\mathcal{G}$; namely, for any object $Y$ of $\mathcal{G}$ Bord, the isomorphism type of the topological vector space $Z(Y)$ is invariant on the path component of $Z$ in the space of field theories. The heuristic reason is that if there is a path of field theories $Z_{t}, t \in[0,1]$, then we have a family $Z_{t}(Y)$ of topological vector spaces parametrized by $[0,1]$. If we interpret that to mean a locally trivial vector bundle, then in particular $Z_{0}(Y)$ and $Z_{1}(Y)$ are isomorphic. This is, in general, an unexpected feature of field theories. For instance, it is conjectured by Stolz and Teichner [13] that supersymmetric Euclidean field theories provide cocycles for certain cohomology theories; in particular, to $1 \mid 1$-dimensional correspond $K$-theory classes. But the dimension of a vector bundle representing a $K$-theory class is not an invariant of the class (only ist virtual dimension), so this should also not be the case at the field theory level.

We choose to deal with this by dropping the local triviality condition and defining an $S$ family of topological (or bornological) vector spaces to be a sheaf of such spaces over $S$ which is a module over the sheaf of smooth functions on $S$. This includes vector bundles over $S$ by associating to a vector bundle its sheaf of sections. It then becomes a fact requiring proof that, under the additional assumption that a field theory is topological, all the families of vector spaces involved turn out to be locally trivial.

\subsection{Homotopy invariance considerations}

One focus in Berwick-Evans and Pavlov [2] is to endow the category of smooth categories (dubbed $C^{\infty}$-categories there) with a model structure. This lets one conclude that the space of field theories is insensitive to fine details in the definitions, as long as everything remains weakly 
equivalent. It also lets one compute with simplified models of the bordism category, since all that matters is that the cofibrancy condition is met, and this is easy in their model structure. We make no attempt to address questions of homotopy meaningfulness in this paper; rather, our focus is on the techniques dealing with the geometric situation.

Lastly, we remark that our bordism category 1-Bord ${ }^{\text {or }}(M)$ possesses an obvious forgetful map to the bordism category of [2]. Since in the model structure on the category of $C^{\infty}$-categories considered there, weak equivalences are just fiberwise equivalences of groupoids, the discussion above shows that this forgetful map is not a weak equivalence in this model structure. Therefore, our result does not follow from that in [2].

\section{Smooth functors and geometric field theories}

Functorial field theories are functors from an appropriate bordism category to a suitable target category. The bordisms in the domain category might come equipped with geometric structures, in a sense to be clarified in this section. After providing examples of geometric structures in Section 3.1 and recalling the language of fibered categories and stacks in Section 3.2, we provide a general definition of "geometries" in Section 3.3. In Section 3.6 we construct the geometric bordism category $\mathcal{G}$ Bord, which is an example of a smooth category, a concept defined in Section 3.4. In the final Section 3.7 we define geometric field theories as "smooth functors" from $\mathcal{G}$ Bord to a suitable smooth target category.

\subsection{Examples of geometries}

The goal of this subsection is to define what we mean by a geometry on smooth manifolds of a fixed dimension $d$, see Definition 3.7. To motivate that abstract definition, we begin by listing well-known structures on manifolds that will be examples of "geometries", and distill their common features into our Definition 3.7.

Examples 3.1. The following are examples of "geometries" on a $d$-manifold $X$ which we would like to capture in an abstract definition:

1. A Riemannian metric or a conformal structure on $X$.

2. A reduction of the structure group of the tangent bundle of $X$ to a Lie group $G$ equipped with a homomorphism $\alpha: G \rightarrow \mathrm{GL}(d)$. More explicitly, such a structure consists of a principal $G$-bundle $P \rightarrow X$ and a bundle map $\alpha_{X}: P \rightarrow \operatorname{Fr}(X)$ to the frame bundle of $X$ (whose total space consists of pairs $(x, f)$ of points $x \in X$ and linear isomorphisms $\left.f: \mathbb{R}^{d} \rightarrow T_{x} X\right)$. The bundle map $\alpha_{X}$ is required to be $G$-equivariant, where the right action of $g \in G$ on $\operatorname{Fr}(X)$ is given by $(x, f) \mapsto(x, f \circ \alpha(g))$. Interesting special cases of reductions of the structure group include the following:

(a) $\mathrm{A} \mathrm{GL}^{+}(d)$-structure on $X$ is an orientation on $X$ (here and in the following three examples, the group $G$ is a subgroup of $\mathrm{GL}(d)$, and $\alpha: G \rightarrow \mathrm{GL}(d)$ is the inclusion map).

(b) An $\mathrm{SL}(d)$-structure on $X$ is a volume form on $X$.

(c) An $\mathrm{O}(d)$-structure on $X$ is a Riemannian metric on $X$.

(d) An $\mathrm{SO}(d)$-structure is Riemannian metric plus an orientation.

(e) A $\operatorname{Spin}(d)$-structure on $X$ is a Riemannian metric plus a spin structure (here $\alpha$ is the composition of the double covering map $\mathrm{Spin}(d) \rightarrow \mathrm{SO}(d)$ and the inclusion $\mathrm{SO}(d) \rightarrow$ $\mathrm{GL}(d))$.

3. A rigid geometry is specified by a $d$-manifold $\mathbb{M}$ (thought of as a "model manifold") and a Lie group $G$ acting on $\mathbb{M}$ (thought of as "symmetries" of $\mathbb{M}$ ). Given this input, a $(G, \mathbb{M})$-structure on $X$ is determined by the following data, which we refer to as a $(G, \mathbb{M})$-atlas for $X$ : 
- an open cover $\left\{X_{i}\right\}_{i \in I}$ of $X$,

- embeddings $\phi_{i}: X_{i} \rightarrow \mathbb{M}$ for $i \in I$ (the charts of the atlas),

- group elements $g_{i j} \in G$ for $X_{i} \cap X_{j} \neq \varnothing$ which make the diagram

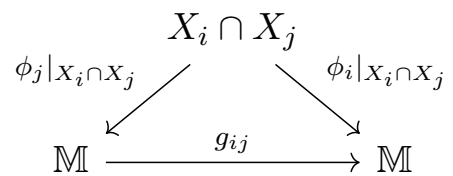

commutative and satisfy a cocycle condition (these are the transition functions for the atlas). Two $(G, \mathbb{M})$-atlases related by refinement of the covers involved define the same $(G, \mathbb{M})$-structure on $X$ (as in the case of smooth atlases for $X$ defining the same smooth structure). Alternatively, analogous to smooth structures, $(G, \mathbb{M})$-structures on $X$ can be defined as maximal $(G, \mathbb{M})$-atlases for $X$. If $X, X^{\prime}$ are two manifolds with $(G, \mathbb{M})$ structure, a morphism between them consists of a smooth map $f: X \rightarrow X^{\prime}$ together with elements $h_{i^{\prime} i} \in G$ for each pair of charts $\left(X_{i}, \phi_{i}\right),\left(X_{i^{\prime}}^{\prime}, \phi_{i^{\prime}}^{\prime}\right)$ with $f\left(X_{i}\right) \subset X_{i^{\prime}}^{\prime}$. such that $\phi_{i^{\prime}}^{\prime} \circ f=h_{i^{\prime} i} \cdot \phi_{i}$ and subject to the coherence condition $h_{j^{\prime} j} \cdot g_{j i}=g_{j^{\prime} i^{\prime}}^{\prime} \cdot h_{i^{\prime} i}$.

Some concrete examples of rigid geometries are as follows:

(a) For $G=\mathrm{SO}(d) \rtimes \mathbb{R}^{d}$, the Euclidean group of isometries of $\mathbb{M}=\mathbb{R}^{d}$, a $(G, \mathbb{M})$-structure on $X$ can be identified with a flat Riemannian metric on $X$.

(b) For $G=\operatorname{Spin}(d) \rtimes \mathbb{R}^{d}$ (where $\operatorname{Spin}(d)$ acts on $\mathbb{R}^{d}$ through $\operatorname{SO}(d)$ ), a $(G, \mathbb{M}$ )-structure on $X$ consists of a flat Riemannian metric together with a spin structure.

(c) For $\mathbb{M}=S^{d}$ and $G=\operatorname{Conf}\left(S^{d}\right)$, the group of conformal transformations of the sphere, a $(G, \mathbb{M})$-structure on $X$ is a conformal structure on $X$.

(d) For $\mathbb{M}=\mathbb{R}^{d}$ and $G=\operatorname{Aff}(d)$, the affine group, a $(G, \mathbb{M})$-structure on $X$ is an affine structure on $X$.

(e) If $\mathbb{M}$ is a simply connected manifold of constant sectional curvature $\kappa$ and isometry group $G$, then a $(G, \mathbb{M})$-structure on $X$ is a Riemannian metric on $X$ of constant curvature $\kappa$.

Rigid geometries as described above are closely related to the notion of pseudogroups, as developed by Cartan. The main difference is that the action of $G$ on $\mathbb{M}$ is not required to be faithful (as, e.g., in the case of the spin group $\operatorname{Spin}(d)$ acting on $\mathbb{R}^{d}$ through $\mathrm{SO}(d)$ ). The above notion was introduced by Stolz and Teichner, with an eye on supersymmetric field theories (see [13, Section 2.5]).

4. A smooth map $X \rightarrow M$ to some fixed manifold $M$.

5. A principal $G$-bundle over $X$ (for a fixed Lie group $G$ ), or a principal $G$-bundle over $X$ with connection.

Remark 3.2. In a physics context, the manifold $X$ is typically the relevant spacetime manifold and the geometry on $X$ is needed for the construction of some field theory. For example, a Riemannian metric on $X$ allows the construction of the scalar field theory whose space of fields is the space $C^{\infty}(X)$ of smooth functions on $X$ and whose action functional is the energy functional given by $S(f):=\int_{X}\|\mathrm{~d} f\|^{2}$ vol (here vol is the volume form determined by the Riemannian metric. A fermionic analog of this field theory consists of fields which are spinors on $X$; its action functional is based on the Dirac operator. The construction of this field theory requires a Riemannian metric and a spin structure on $X$, i.e., a reduction of the structure group to $\operatorname{Spin}(d)$. 
In many of the Examples 3.1, the geometries on a fixed $d$-manifold $X$ form just a set (in particular, in the cases $(1),(2 a),(2 b),(2 c),(2 d),(3 a),(3 c),(3 d),(3 e)$ and $(4))$. In other cases, e.g., $(2 e),(3 b),(5)$, there is more going on: these geometric structures can be interpreted as the objects of a groupoid which contains non-identity morphisms. For example, for a fixed group $G$, the principal $G$-bundles $P \rightarrow X$ over $X$ form a groupoid, with the morphisms from $P$ to $P^{\prime}$ being the $G$-equivariant maps that commute with the projection maps to $X$ (this is Example 3.1(5)).

This suggests to think of a $\mathcal{G}$-structure on $X$ as an object of a groupoid $\mathcal{G}(X)$ associated to $X$ (which might be discrete in the sense that the only morphisms in that groupoid are identity morphisms, as in our examples $(1),(2 a),(2 b),(2 c),(2 d),(3 a),(3 c),(3 d),(3 e)$ and $(4))$. A crucial feature of all our Examples 3.1 is that the data of a geometry is local in $X$ in a sense to be made precise. For example, a Riemannian metric on $X$ is determined by prescribing a Riemannian metric $g_{i}$ on each open subset $X_{i}$ belonging to an open cover $\left\{X_{i}\right\}_{i \in I}$ of $X$ in such a way that these metrics $g_{i}, g_{j}$ coincide on the intersection $X_{i} \cap X_{j}$. In other words, the Riemannian metrics on $X$ form a sheaf. The same statement is true in our other examples of geometric structures $\mathcal{G}(X)$, where the groupoid $\mathcal{G}(X)$ is discrete.

In the case of non-discrete groupoids, for example if $\mathcal{G}(X)$ is the groupoid of principal $G$ bundles over $X$ (as in Example 3.1(5)), it is still true that $\mathcal{G}(X)$ is local in $X$, but it is harder to formulate what that means. The idea is that for any open cover $\left\{X_{i}\right\}_{i \in I}$ of $X$ the groupoids associated to intersections of the $X_{i}$ determine the groupoid $\mathcal{G}(X)$ of principal bundles over $X$, up to equivalence. This is expressed by saying the groupoids $\mathcal{G}(X)$ form a stack on the site of manifolds. For the precise definition of stack we refer the reader to Vistoli's survey paper [15, cf. Definition 4.6], but it should be possible to follow our discussion below without prior knowledge of stacks. In fact, we hope that the following might motivate a reader not already familiar with stacks to learn about them.

\subsection{Digression on stacks}

Our first example of a stack will be the stack Vect of vector bundles. We note here the relevant structures.

- For a fixed manifold $X$ let $\operatorname{Vect}(X)$ be the category of whose objects are smooth vector bundles $E \rightarrow X$ over $X$ and whose morphisms from $E \rightarrow X$ to $E^{\prime} \rightarrow X$ are smooth maps $F: E \rightarrow E^{\prime}$ which commute with the projection to $X$ and whose restriction $F_{x}: E_{x} \rightarrow E_{x}^{\prime}$ to the fibers over $x \in X$ is a linear map for each $x \in X$.

- Let Vect be the category whose objects are vector bundles $E \rightarrow X$ over some manifold $X$ and whose morphisms from $E \rightarrow X$ to $E^{\prime} \rightarrow X^{\prime}$ are pairs of smooth maps $(\hat{f}, f)$ for which the diagram

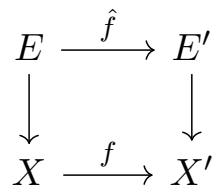

is commutative, and the restriction $\hat{f}_{x}: E_{x} \rightarrow E_{f(x)}^{\prime}$ of $\hat{f}$ to the fiber over $x$ is linear for all $x \in X$. Abusing notation, we often simply write $\phi: E \rightarrow E^{\prime}$ for such a morphism $\phi=(\hat{f}, f)$.

There is an obvious functor $p$ : Vect $\rightarrow$ Man to the category of smooth manifolds that sends a vector bundle $E$ to its base space. The category $\operatorname{Vect}(X)$ of vector bundles over $X$ is the fiber of $p$, i.e., the subcategory of Vect consisting of all objects whose image under $p$ is $X$ and all 
morphisms of Vect whose image under $p$ is the identity of $X$. The functor $p$ : Vect $\rightarrow$ Man has two interesting properties:

Existence of cartesian lifts. Given a smooth map $f: X \rightarrow X^{\prime}$ and a vector bundle $E^{\prime}$ over $X^{\prime}$, we can form the pullback bundle $E:=f^{*} E^{\prime}$ over $X$, which is the domain of a tautological morphism $\phi=(\hat{f}, f): E \rightarrow E^{\prime}$. The vector bundle map $\phi$ has the property that, for each $x \in X$, the linear map of fibers $\hat{f}_{x}: E_{x} \rightarrow E_{f(x)}^{\prime}$ is an isomorphism. Morphisms with this property are called cartesian, and the vector bundle morphism $\phi: E=f^{*} E^{\prime} \rightarrow E$ is also referred to as the cartesian lift of the morphism $f: X \rightarrow X^{\prime}$.

While the characterization of cartesian vector bundle morphisms $\phi: E \rightarrow E^{\prime}$ as those which restrict to fiberwise isomorphisms is hands-on and concrete, it is more common to characterize them by the universal property we describe now. The advantage is that universal properties make sense in any category.

A vector bundle morphism $\phi: E^{\prime} \rightarrow E$ is cartesian if, for any vector bundle map $\phi^{\prime \prime}: E^{\prime \prime} \rightarrow E$ and any map $f^{\prime}: p\left(E^{\prime \prime}\right) \rightarrow p\left(E^{\prime}\right)$ such that $p\left(\phi^{\prime \prime}\right)=p(\phi) \circ f^{\prime}$, there exists a unique vector bundle map $\phi^{\prime}: E^{\prime \prime} \rightarrow E^{\prime}$ with $p\left(\phi^{\prime}\right)=f^{\prime}$. This property can be expressed succinctly by saying that, given a commutative diagram consisting of the solid arrows in the diagram below, there exists a unique morphism $\phi^{\prime}$ indicated by the dashed arrow that makes the whole diagram commutative. Here, $X=p(E), f=p(\phi)$, etc., and by commutativity of the squares we mean that applying the functor $p$ : Vect $\rightarrow$ Man to the top morphism in Vect gives the bottom morphism in Man:

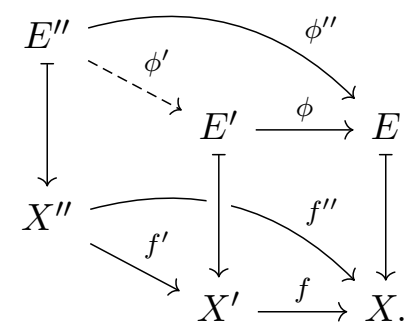

Descent property. Let $f_{i}: X_{i} \rightarrow X$ be a collection of morphisms in Man that is a cover of $X$ in the sense that all the $f_{i}$ are open embeddings and the union of the images $f_{i}\left(X_{i}\right)$ is all of $X$. Then the category $\operatorname{Vect}(X)$ can be reconstructed, up to equivalence, from the categories $\operatorname{Vect}\left(X_{i}\right), \operatorname{Vect}\left(X_{i} \cap X_{j}\right)$, and $\operatorname{Vect}\left(X_{i} \cap X_{j} \cap X_{k}\right)$ and the restriction functors between them. More precisely, from the diagram given by these categories and the restriction functors between them one can construct the descent category $\operatorname{Vect}\left(\left\{X_{i} \rightarrow X\right\}\right)$ associated to the cover $\left\{X_{i} \rightarrow X\right\}$ (see [15, Section 4.1.2]) and a restriction functor

$$
\operatorname{Vect}(X) \longrightarrow \operatorname{Vect}\left(\left\{X_{i} \rightarrow X\right\}\right),
$$

which is an equivalence.

It turns out that the existence of pullbacks and the descent property can be formulated quite generally for functors $p: \mathcal{F} \rightarrow \mathcal{S}$ as follows.

Definition 3.3 (cartesian morphism, fibration, prestack). Let $p: \mathcal{F} \rightarrow \mathcal{S}$ be a functor. A morphism $\phi$ in the category $\mathcal{F}$ is cartesian if it satisfies the universal property expressed by the diagram (3.1); see also [15, Definition 3.1]. The functor $p: \mathcal{F} \rightarrow \mathcal{S}$ is called a Grothendieck fibration and $\mathcal{F}$ a category fibered over $\mathcal{S}$ if, for any morphism $f: X^{\prime} \rightarrow X$ in $\mathcal{S}$ and object $E \in \mathcal{F}$ with $p(E)=X$, there is a cartesian morphism $\phi: E^{\prime} \rightarrow E$ with $p(\phi)=f$; see also [15, Definition 3.1]. We say that a fibration $\mathcal{F} \rightarrow \mathcal{S}$ is a prestack if every morphism of $\mathcal{F}$ is cartesian.

Remark 3.4. That $\mathcal{F} \rightarrow \mathcal{S}$ is a prestack means that $\mathcal{F} \rightarrow \mathcal{S}$ is fibered in groupoids, meaning that the subcategory $\mathcal{F}(S)$ of $\mathcal{F}$ lying over $\operatorname{id}_{S}$ is a groupoid for every object $S$ of $\mathcal{S}$. Conversely, it 
turns out that a category fibered in groupoids is automatically a prestack [15, Proposition 3.22]. To any prestack corresponds a presheaf of groupoids on $\mathcal{S}$, sending $S$ to $\mathcal{F}(S)$. This sheaf depends on a choice of pullback for every object $F$ of $\mathcal{F}$ along every morphism $f: S \rightarrow T$ in $\mathcal{S}$ (i.e., a cartesian arrow $\phi$ in $\mathcal{F}$ with target $F$ and $p(\phi)=f$ ), but it is unique up to unique isomorphism.

As discussed above, for the functor $p$ : Vect $\rightarrow$ Man the cartesian morphisms in Vect are the vector bundle morphisms $\phi: E^{\prime} \rightarrow E$ that restrict to isomorphisms on fibers. Moreover, given a smooth map $f: X^{\prime} \rightarrow X$ between manifolds and a vector bundle $E$ over $X$, the tautological bundle map $\phi$ from the pullback bundle $E^{\prime}:=f^{*} E$ to $E$ is a cartesian lift of $f$. Hence $p$ : Vect $\rightarrow$ Man is a Grothendieck fibration; in other words, Vect is fibered over Man.

The discussion of the descent property for $p$ : Vect $\rightarrow$ Man above was based on the definition of a cover $\left\{X_{i} \rightarrow X\right\}$ of a manifold $X$. So, before discussing descent in a general category $\mathcal{S}$, we need to clarify what is meant by a "cover" of an object $X$ of $\mathcal{S}$.

Definition 3.5 (cover, Grothendieck topology, site [15, Definition 2.24]). Let $\mathcal{S}$ be a category. A Grothendieck topology on $\mathcal{S}$ is the assignment, to each object $X$ of $\mathcal{S}$, of a collection of sets of morphisms $\left\{X_{i} \rightarrow X\right\}$, called covers of $X$, so that the following conditions are satisfied:

1. If $Y \rightarrow X$ is an isomorphism, the set $\{Y \rightarrow X\}$ is a cover.

2. If $\left\{X_{i} \rightarrow X\right\}$ is a cover and $Y \rightarrow X$ is any morphism, then the pullbacks $X_{i} \times_{X} Y$ exist, and the collection of projections $\left\{X_{i} \times_{X} Y \rightarrow Y\right\}$ is a cover.

3. If $\left\{X_{i} \rightarrow X\right\}$ is a cover and, for each index $i$, we have a cover $\left\{Y_{i j} \rightarrow X_{i}\right\}$, the collection of composites $\left\{Y_{i j} \rightarrow X_{i} \rightarrow X\right\}$ is a cover of $X$ (here $j$ varies in a set depending on $i$ ).

A category equipped with a Grothendieck topology is called a site.

Definition 3.6 (descent category, stack). If $\mathcal{F}$ is a prestack over $\mathcal{S}$, then there is a descent category $\mathcal{F}\left(\left\{X_{i} \rightarrow X\right\}\right)$ associated to a collection $\left\{X_{i} \rightarrow X\right\}$ of morphisms in $\mathcal{S}$ (see [15, Section 4.1.2]) and an associated functor

$$
\mathcal{F}(X) \longrightarrow \mathcal{F}\left(\left\{X_{i} \rightarrow X\right\}\right)
$$

A prestack $\mathcal{F} \rightarrow \mathcal{S}$ over a site $\mathcal{S}$ is called a stack, if, for every cover $\left\{X_{i} \rightarrow X\right\}$ of every object $X$ in $\mathcal{S}$, the functor (3.2) is an equivalence.

For a stack, the groupoids $\mathcal{F}\left(X_{i}\right)$ associated to the patches $X_{i}$, together with transition data on double and triple intersections, determine $\mathcal{F}(X)$. In fact, this definition makes sense for general fibered categories, not only those fibered in groupoids (i.e., prestacks). In this paper, we will use the unqualified term stack only for those stacks which are fibered in groupoids, and say stack of categories in the general case, when not all morphisms need to be cartesian. Similar to the case of sheaves, to any prestack, there is a canonically associated stack, its stackification [6, p. 18].

Let $\operatorname{Man}^{d}$ be the category of $d$-dimensional manifolds and smooth maps. We will always consider the Grothendieck topology on $\mathrm{Man}^{d}$ of jointly surjective open embeddings. Precisely, a collection $\left\{X_{i} \rightarrow X\right\}$ is a cover, if each map $X_{i} \rightarrow X$ is an open embedding and the images of the $X_{i}$ in $X$ cover $X$.

Definition 3.7 (geometry, preliminary!). A geometry on d-manifolds is a stack $\mathcal{G} \rightarrow \operatorname{Man}^{d}$ on the site $\mathrm{Man}^{d}$ of manifolds of dimension $d$.

We end our digression on stacks with a few more general remarks. 
Definition 3.8 (fibered functors, base-preserving natural transformations). Let $\mathcal{F}, \mathcal{G} \rightarrow \mathcal{S}$ be two Grothendieck fibrations. A functor $H: \mathcal{F} \rightarrow \mathcal{G}$ is called a fibered functor if it commutes strictly with the projections to $\mathcal{S}$ and sends cartesian morphisms to cartesian morphisms. A natural transformation $\xi: H \rightarrow K$ between fibered functors is base-preserving if, for any object $x \in \mathcal{F}$, the morphism $\xi_{x}: H(x) \rightarrow K(x)$ maps to an identity morphism in $\mathcal{S}$.

Definition 3.9 (categories of (pre-)stacks). For each site $\mathcal{S}$, we get a 2-category PSt $\mathrm{S}_{\mathcal{S}}$ of Grothendieck fibrations, fibered functors and base-preserving natural transformations. The full subcategory of stacks will be denoted by $\mathrm{St}_{\mathcal{S}}$. We will omit the subscript when $\mathcal{S}=$ Man is the site of smooth manifolds.

\subsection{Geometries in families}

The preliminary Definition 3.7 satisfactorily captures the contravariance and locality aspects of a geometric structure. However, as discussed in Section 2.3, it is crucial to work with families of smooth manifolds. In particular, we need to talk about geometric structures on families of $d$ manifolds. This is formalized in Definition 3.12 below by replacing the category $\mathrm{Man}^{d}$ in the preliminary definition by the category $\mathrm{Fam}^{d}$ of families of $d$-dimensional manifolds, equipped with a suitable Grothendieck topology.

Definition 3.10 (families of manifolds). Denote by Fam the category of families of smooth manifolds, where an object, typically denoted by $X / S$, is simply a submersion $X \rightarrow S$, and a morphism $X^{\prime} / S^{\prime} \rightarrow X / S$ is a fiberwise open embedding

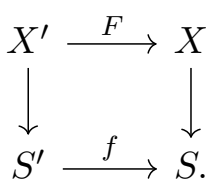

By that we mean that the diagram commutes and the map $X^{\prime} \rightarrow S^{\prime} \times{ }_{S} X$ is an open embedding. We denote by $\mathrm{Fam}^{d}$ the subcategory of families with $d$-dimensional fibers.

To turn Fam into a site, we declare a cover of the object $X / S \in$ Fam to be a collection of morphisms $\left\{X_{i} / S_{i} \rightarrow X / S\right\}_{i \in I}$ such that the images of the $X_{i}$ form an open cover of $X$. This satisfies the axioms of a Grothendieck topology: it is clear that covers of covers determine a cover, and we check the existence and stability of base changes (condition (2) of Definition 3.5) in the following lemma.

Lemma 3.11. If $\left\{X_{i} / S_{i} \rightarrow X / S\right\}$ is a cover and $Y / T \rightarrow X / S$ is any morphism, then the fiber products $\left(X_{i} / S_{i}\right) \times_{(X / S)}(Y / T)$ exist and determine a cover of $Y / T$.

Proof. Write $Y_{i}=X_{i} \times_{X} Y, T_{i}=S_{i} \times_{S} T$. Both are manifolds since the maps $X_{i} \rightarrow X$ and $S_{i} \rightarrow S$ are submersions: The first by the requirement that the $X_{i}$ form an open cover of $X$; to see that the latter is, observe that the composition $X_{i} \rightarrow X \rightarrow S$ is a submersion, which equals $X_{i} \rightarrow S_{i} \rightarrow S$, hence $S_{i} \rightarrow S$ must be a submersion, too. We now have a diagram as follows:

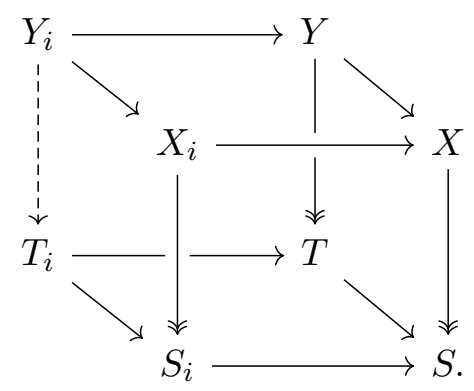


The dashed map is obtained by the cartesian property of the bottom face (containing $S_{i}$ and $T$ ), which implies that all faces of the cube commute. To see that $Y_{i} \rightarrow T_{i}$ is a submersion, let $\left(s_{i}, t\right) \in T_{i}=S_{i} \times_{S} T$ be a point that is the image of the point $\left(x_{i}, y\right) \in Y_{i}=X_{i} \times_{X} Y$. Further, consider a tangent vector to $\left(s_{i}, t\right)$, represented by a pair of paths $\left(\gamma_{S_{i}}, \gamma_{T}\right)$, where $\gamma_{S_{i}}: I \rightarrow S_{i}$, $\gamma_{S_{i}}(0)=s_{i}, \gamma_{T}: I \rightarrow T, \gamma_{T}(0)=t$ are paths that coincide after passing to $S$. Since $Y$ is a submersion, we can find a lift $\gamma_{Y}: I^{\prime} \rightarrow Y$ (with $I^{\prime} \subset I$ ) of $\gamma_{T}$ with $\gamma_{Y}(0)=y$. Let $\tilde{\gamma}_{X}$ be the image of this lift to $X$. By construction, $\gamma_{X}(0)$ lies in the image of the map $X_{i} \rightarrow X$, hence (because $X_{i}$ is an open embedding), we can find a path $\gamma_{X_{i}}: I^{\prime \prime} \rightarrow X_{i}$ (with $I^{\prime \prime} \subset I^{\prime}$ ) that maps to $\gamma_{X}$. Now $\left(\gamma_{X_{i}}, \gamma_{Y}\right)$ represents a tangent vector to $\left(x_{i}, y\right)$ that maps to $\left(s_{i}, t\right)$ under the map $Y_{i} \rightarrow S_{i}$.

To see that $Y_{i}$ is a fiberwise open embedding, we have to show that $Y_{i} \rightarrow T_{i} \times_{T} Y$ is an open embedding. To see that it is injective, let $\left(x_{i}, y\right)$ and $\left(x_{i}^{\prime}, y^{\prime}\right) \in X_{i} \times_{X} Y=Y_{i}$ be two points that are mapped to the same point in $T_{i} \times_{T} \times Y$. First, it follows that $x_{i}$ and $x_{i}^{\prime}$ are mapped to the same point in $X$; since $X_{i} \rightarrow X$ is an embedding, we have that $x_{i}=x_{i}^{\prime}$. Secondly, since $\left(x_{i}, y\right)$ and $\left(x_{i}^{\prime}, y^{\prime}\right)$ coincide in $T_{i} \times_{T} Y$, we must have $y=y^{\prime}$. This shows injectivity. The map $Y_{i}=X_{i} \times_{X} Y \rightarrow T_{i} \times_{T} Y$ is a submersion by a similar argument as above. To see that it is also an immersion, take a tangent vector represented by a tuple $\left(\gamma_{X_{i}}, \gamma_{Y}\right)$ of curves $\gamma_{X_{i}}: I \rightarrow X_{i}$ and $\gamma_{Y}: I \rightarrow Y$ that coincide after passing to $X$. Then the pushforward of this tangent vector is represented by $\left(\gamma_{S_{i}}, \gamma_{Y}\right)$, where $\gamma_{S_{i}}$ is the image of $\gamma_{X_{i}}$ under the map $X_{i} \rightarrow S_{i}$. Assume that this pushforward is zero, i.e., $\dot{\gamma}_{S_{i}}(0)=0$ and $\dot{\gamma}_{Y}(0)=0$. Then because $\gamma_{X_{i}}$ and $\gamma_{Y}$ are mapped to the same curve $\gamma_{X}: I \rightarrow X$ and $X_{i} \rightarrow X$ is an embedding, we have that also $\dot{\gamma}_{X_{i}}(0)=\dot{\gamma}_{X}(0)=\dot{\gamma}_{Y}(0)=0$. Hence the tangent vector represented by $\left(\gamma_{X_{i}}, \gamma_{Y}\right)$ is zero as well and $Y_{i} \rightarrow T_{i} \times_{T} Y$ is an immersion. In total, we obtain that it is an open embedding.

It only remains to see that $Y_{i} / T_{i}$ has the universal property of the fiber product $\left(X_{i} / S_{i}\right) \times(X / S)$ $(Y / T)$. In other words, given an arbitrary family $Z / U$ and maps $Z / U \rightarrow X_{i} / S_{i}, Z / U \rightarrow Y / T$ which agree on $X / S$, there exists a unique morphism $Z / U \rightarrow Y_{i} / T$ making the diagram

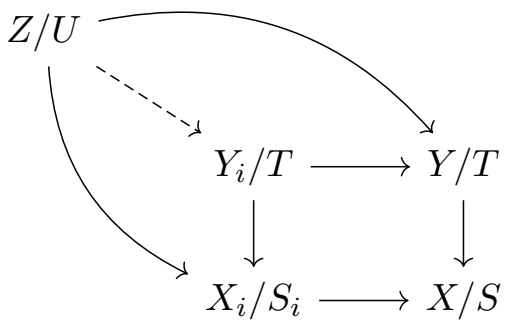

commute. By the cartesian property of two of the squares of the cube (3.3), there exist unique maps $Z \rightarrow Y_{i}$ and $U \rightarrow T_{i}$. We claim that these determine a morphism in Fam, that is, the diagram

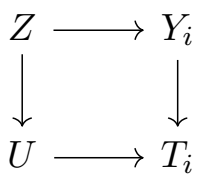

commutes and the top map is a fiberwise open embedding. Both maps $Z \rightarrow T_{i}$ agree when postcomposed with $T_{i} \rightarrow S_{i}$ respectively $T_{i} \rightarrow T$, so they agree by the universal property of $T_{i}=S_{i} \times_{S} T$. The map $Z \rightarrow Y_{i}$ is an a fiberwise open embedding because so is its composition $Z \rightarrow Y_{i} \rightarrow Y$ with another fiberwise open embedding. Hence this morphism fits in as the dashed morphism in (3.4). By uniqueness of the maps $Z \rightarrow Y_{i}$ and $U \rightarrow T_{i}$, this is the unique morphism with this property.

The above lemma shows that our notion of cover defines a Grothendieck topology, which turns Fam into a site. By restricting to those families $X / S$, where the fibers $X_{s}, s \in S$ are 
all $d$-dimensional, we get a subcategory, $\mathrm{Fam}^{d}$. Since our covers do not mix fiber dimensions, restricting to $d$-dimensional covers turns also $\mathrm{Fam}^{d} \subset$ Fam into a site. This allows to talk about sheaves and stacks on $\mathrm{Fam}^{d}$.

We are now ready to give the main definition of this section.

Definition 3.12 (geometry). A $d$-dimensional geometry is a stack $\mathcal{G}$ on the site $\mathrm{Fam}^{d}$ of families of manifolds with $d$-dimensional fibers. By an $S$-family of $\mathcal{G}$-manifolds we will mean a family $X / S \in \mathrm{Fam}^{d}$ together with an object of $\mathcal{G}(X / S)$.

To each family $X / S$ are associated a natural relative tangent bundle $T(X / S)=\operatorname{Ker}(T X \rightarrow$ $T S)$ and variations: the relative cotangent bundle $T^{\vee}(X / S)=\operatorname{Coker}\left(T^{\vee} S \rightarrow T^{\vee} X\right)$, their tensor powers, etc. For emphasis, we sometimes call their sections fiberwise vector fields, differential forms, etc. This allows us to define fiberwise versions (or "families") of many familiar structures, such as Riemannian metrics, symplectic and complex structures, connections on a principal bundle, and so on. For instance, a Riemannian metric on $X / S$ is a positive-definite section of the second symmetric power of $T^{\vee}(X / S)$. A family of connections on a vector bundle $V \rightarrow X$ is a differential operator $\nabla: C^{\infty}(V) \rightarrow C^{\infty}\left(T^{\vee}(X / S) \otimes V\right)$ satisfying a version of the Leibniz rule involving the fiberwise exterior derivative $\mathrm{d}: C^{\infty}(X) \rightarrow \Omega^{1}(X / S)$. Thus, $\nabla$ allows us to perform parallel transport only along the fibers of the submersion $X \rightarrow S$.

It is now mostly straightforward to adapt Examples 3.1 to geometries in families. We spell this out in two cases.

Example 3.13 (families over a manifold $M$ ). Any manifold $M$ represents a geometry on $d$ dimensional manifolds ( $d$ arbitrary): $S$-families are manifolds $X / S$ together with a smooth function $\gamma: X \rightarrow M$, and morphisms $\left(X^{\prime} / S^{\prime}, \varphi^{\prime}\right) \rightarrow(X / S, \varphi)$ are maps $F: X^{\prime} / S^{\prime} \rightarrow X / S$ such that $\gamma^{\prime}=\gamma \circ F$. This in fact defines a sheaf on Fam $^{d}$.

Example 3.14 (rigid geometries). We recast the family version of rigid geometries [13, Section 2.5] in the language of this paper. As in Example 3.1(3), fix a $d$-dimensional model manifold $\mathbb{M}$ and a Lie group $G$ acting on it. Then we define $\mathcal{G}$, the stack of $(G, \mathbb{M})$-atlases, to be the stackification of the prestack on Fam $^{d}$ described as follows:

1. An object lying over $X / S$ is given by a fiberwise open embedding

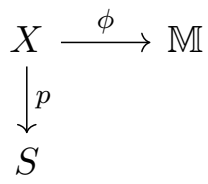

or, in other words, an open embedding $(p, \phi): X \rightarrow S \times \mathbb{M}$.

2. A morphism lying over $(f, F): X^{\prime} / S^{\prime} \rightarrow X / S$ is given by a map $g: S^{\prime \prime} \rightarrow G$ such that the diagram

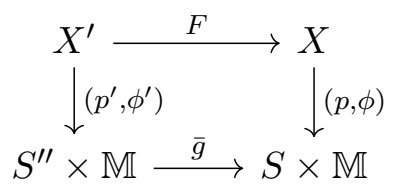

commutes, where $S^{\prime \prime}=p^{\prime}\left(X^{\prime}\right) \subset S^{\prime}$ and $\bar{g}:(s, x) \mapsto(f(s), g(s) \cdot x)$ is the map induced by $f$ and the action by $g$.

The composition of morphisms is determined by composition of the $\bar{g}$. Note that every morphism is cartesian. 
The usual stackification procedure [6, p. 18] exactly recovers the more concrete definition of a rigid geometry given in [13, Definition 2.33]: A section of the stack $\mathcal{G}$ over $X / S$, which we call a $(G, \mathbb{M})$-atlas, is given by the following data: (1) a cover $\left\{X_{i} / S_{i} \rightarrow X / S\right\}_{i \in I}$, (2) fiberwise embeddings $\phi_{i}: X_{i} \rightarrow \mathbb{M}$ for each $i \in I$ (the charts of the atlas), and (3) transition functions $g_{i j}: S_{i} \times_{S} S_{j} \supset p\left(X_{i} \times_{X} X_{j}\right) \rightarrow G$ relating appropriate restrictions of $\phi_{i}$ and $\phi_{j}$, and satisfying a cocycle condition. Morphisms between atlases based on the same cover $\left\{X_{i} / S_{i}\right\}_{i \in I}$ are given by collections of maps $h_{i}: S_{i} \rightarrow G, i \in I$, which interpolate the charts $X_{i} \rightarrow \mathbb{M}$. Moreover, atlases related by a refinement of covers must be declared equivalent; this is taken care of by the stackification machinery.

\subsection{Simplicial prestacks and smooth categories}

It is well known (see, e.g., [11, Section 2]) that a simplicial set $\mathcal{C}: \Delta^{\mathrm{op}} \rightarrow$ Set is equivalent to the nerve of a category if, and only if, the Segal maps

$$
\left(s_{n}^{*}, \ldots, s_{1}^{*}\right): \mathcal{C}_{n} \longrightarrow \mathcal{C}_{1} \times \mathcal{C}_{0} \cdots \times \times_{\mathcal{C}_{0}} \mathcal{C}_{1}
$$

are bijections for $n \geq 2$. Here, $s_{i}:[1] \rightarrow[n], i=1, \ldots, n$, is the morphism sending $0 \mapsto i-1$ and $1 \mapsto i$, and fiber products are taken over the maps $d_{0}^{*}, d_{1}^{*}: \mathcal{C}_{1} \rightarrow \mathcal{C}_{0}$ induced by the two maps $d_{0}, d_{1}:[0] \rightarrow[1]$. (For references, see, e.g., $\left.[9,11].\right)$

This observation allows us to internalize the notion of a category in other ambient (higher) categories. In this paper, we would like to talk about categories endowed with a notion of "smooth families" of objects and morphisms. Thus, we take as ambient the 2-category PSt of prestacks on Man.

Definition 3.15 (simplicial prestack). A simplicial prestack (on manifolds) is a pseudofunctor $\mathcal{C}: \Delta^{\mathrm{op}} \rightarrow$ PSt.

Remark 3.16. Here the simplex category $\Delta$ is regarded as a 2-category with only trivial 2morphisms, and all constructions are performed in the realm of bicategories. That $\mathcal{C}$ is a pseudofunctor then means that for two composable morphisms $\eta, \kappa$ in $\Delta$, the induced morphisms of stacks $\kappa^{*} \eta^{*}$ and $(\eta \kappa)^{*}$ agree only up to a coherent natural isomorphism, which is part of the data of $\mathcal{C}$.

A smooth category will be a simplicial prestack satisfying suitable conditions. Before introducing them, we fix some terminology. Condition (2) below assures that the simplicial set $n \mapsto h_{0} \mathcal{C}_{n}(S)$ is equivalent to the nerve of a category $C$ (where $h_{0} \mathcal{C}_{n}(S)$ is the set of isomorphism classes of objects in $\mathcal{C}_{n}(S)$ ); we call an object of $\mathcal{C}_{1}(S)$ an equivalence if it represents an invertible morphism in $C$.

Definition 3.17 (smooth category). A smooth category $\mathcal{C}$ is a simplicial stack $\mathcal{C}: \Delta^{\mathrm{op}} \rightarrow \mathrm{St}$ such that

(1) the Segal maps (3.5) are equivalences of stacks, and

(2) the degeneracy map $\mathcal{C}_{0} \rightarrow \mathcal{C}_{1}$ gives an equivalence of the domain with the full substack of equivalences in $\mathcal{C}_{1}$.

We will refer to morphisms and 2-morphisms between smooth categories as smooth functors and smooth natural transformations, respectively.

The above conditions are modeled on complete Segal spaces, which extends the nerve construction explained above, to give a model for $(\infty, 1)$-categories (see [9] for further details). Condition (1) is the Segal condition, (2) is the completeness condition. In other words, smooth stacks are complete Segal objects in St. 
Remark 3.18. The fiber products appearing in the definition (which are taken using $d_{0}^{*}, d_{1}^{*}$ : $\left.\mathcal{C}_{1} \rightarrow \mathcal{C}_{0}\right)$ are in the bicategorical sense, that is, they are what is sometimes called a homotopy fiber product.

Example 3.19 (smooth categories from smooth stacks). Our most interesting examples of smooth categories will be the geometric bordism categories constructed below. However, to get the first examples, we now provide a way to construct a smooth category from a smooth stack. This is a version of Rezk's classification diagram construction [9].

Let $\mathcal{C}$ be a stack of categories (so that $\mathcal{C}(S)$ does not need to be a groupoid). In our applications, $\mathcal{C}$ will be the stack of vector bundles or a stack of sheaves of $C^{\infty}$-modules as in Section 5.2. We then construct a smooth category $\mathcal{C}_{\bullet}$ from this input as follows.

Objects of $\mathcal{C}_{n}$ lying over $S \in$ Man are tuples $\left(C_{n}, \ldots, C_{0} ; f_{n}, \ldots, f_{1}\right)$, where the $C_{j}$ are objects of $\mathcal{C}(S)$ and $f_{j}: C_{j-1} \rightarrow C_{j}$ are morphisms in $\mathcal{C}(S)$ (i.e., morphisms in $\mathcal{C}$ covering the identity on $S)$. Morphisms from an object $\left(C_{n}, \ldots, C_{0} ; f_{n}, \ldots, f_{1}\right)$ over $S$ to an object $\left(C_{n}^{\prime}, \ldots, C_{0}^{\prime} ; f_{n}^{\prime}, \ldots, f_{1}^{\prime}\right)$ over $T$ covering $f: S \rightarrow T$ are tuples $\left(\alpha_{n}, \ldots, \alpha_{0}\right)$, where $\alpha_{j}: C_{j} \rightarrow C_{j}^{\prime}$ are cartesian arrows covering $f$ such that the diagram

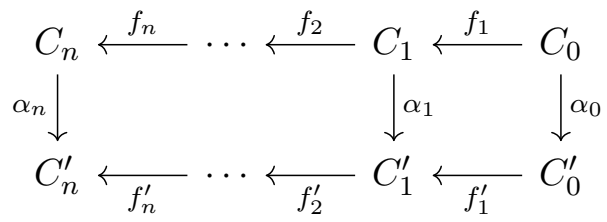

commutes. The simplicial structure of $\mathcal{C} \bullet$ is so that face maps perform composition of morphisms and degeneracies insert identities. More explicitly, a morphism $\kappa:[n] \rightarrow[m]$ in $\Delta$ induces the functor $\kappa^{*}: \mathcal{C}_{m} \rightarrow \mathcal{C}_{n}$ with

$$
\kappa^{*}\left(V_{m}, \ldots, V_{0} ; f_{m}, \ldots, f_{1}\right)=\left(V_{\kappa(n)}, \ldots, V_{\kappa(0)} ; f_{n}^{\prime}, \ldots, f_{1}^{\prime}\right),
$$

where

$$
f_{j}^{\prime}= \begin{cases}f_{\kappa(j)} \cdots f_{\kappa(j-1)+1} & \text { if } \kappa(j-1)<\kappa(j), \\ \text { id } & \text { otherwise }\end{cases}
$$

and

$$
\kappa^{*}\left(\alpha_{m}, \ldots, \alpha_{0}\right)=\left(\alpha_{\kappa(n)}, \ldots, \alpha_{\kappa(0)}\right) .
$$

This gives a (strict) functor $\mathcal{C}_{\bullet}: \Delta^{\mathrm{op}} \rightarrow \mathrm{St}_{\mathrm{Man}}$, and it is obvious that it satisfies the Segal condition.

Definition 3.20 (strictness). We say that a simplicial prestack $\mathcal{C}$ is strict if it is a strict functor, that is, the natural isomorphisms $\kappa^{*} \eta^{*} \cong(\eta \kappa)^{*}$ are all identities. We say that a smooth functor between two strict smooth categories is strict if it commutes on the nose with the structure maps in $\Delta$, as a natural transformation of strict functors.

The following is an easy structure result for the examples just constructed, whose proof we omit.

Lemma 3.21. Let $\mathcal{B}$ be a strict simplicial prestack and let $\mathcal{V}$ be a smooth category of the type constructed in Example 3.19. Let $Z, Y: \mathcal{B} \rightarrow \mathcal{V}$ be two strict functors. Then the map

$$
\operatorname{Nat}(Z, Y) \longrightarrow \operatorname{Nat}\left(Z_{0}, Y_{0}\right)
$$

that restricts a smooth natural transformation to the corresponding 2-morphisms of stacks at simplicial level zero is injective. Moreover, the map

$$
\operatorname{Nat}(Z, Y) \longrightarrow \operatorname{Nat}\left(Z_{1}, Y_{1}\right)
$$

that restricts to simplicial level one is an isomorphism. 


\subsection{Symmetric monoidal structures}

To talk about field theories, we need to endow our smooth categories with symmetric monoidal structures. This requires first to define symmetric monoidal stacks.

Definition 3.22 (monoidal fibered category). A symmetric monoidal fibered category is a fibered category $\mathcal{F} \rightarrow \mathcal{S}$ with a (fibered) tensor product functor

$\otimes: \mathcal{F} \times{ }_{\mathcal{S}} \mathcal{F} \longrightarrow \mathcal{F}$,

a (fibered) unit functor $\epsilon: \mathcal{S} \rightarrow \mathcal{F}$ (where $\mathcal{S} \rightarrow \mathcal{S}$ denotes the trivial fibered category), together with a collection of natural transformations (an associator $\alpha:(X \otimes Y) \otimes Z \rightarrow X \otimes(Y \otimes Z)$, left and right unitors $\lambda: 1 \otimes X \rightarrow X, \rho: X \otimes 1 \rightarrow X$ and a braiding $B: X \otimes Y \rightarrow Y \otimes X)$. These data are required to be compatible in the sense that they turn each fiber category $\mathcal{F}(S)$ into a symmetric monoidal category. A symmetric monoidal stack is a symmetric monoidal fibered category that is also a stack.

Symmetric monoidal smooth stacks together with (strong) symmetric monoidal functors and natural transformations form a bicategory which we shall denote $\mathrm{St}_{\mathrm{Man}}^{\otimes}$.

Definition 3.23 (symmetric monoidal smooth category). A symmetric monoidal structure on a simplicial prestack $\mathcal{C}: \Delta^{\mathrm{op}} \rightarrow \mathrm{St}_{\mathrm{Man}}$ is a lift of $\mathcal{C}$ to a pseudofunctor $\Delta^{\mathrm{op}} \rightarrow \mathrm{St}_{\text {Man }}^{\otimes}$. Symmetric monoidal smooth functors and natural transformations are likewise defined as 1- and 2-morphisms of simplicial objects in symmetric monoidal stacks. A symmetric monoidal smooth category is a smooth category (Definition 3.17) with a symmetric monoidal structure.

Example 3.24. If, in Example 3.19, we start with a symmetric monoidal smooth stack as input, the result will naturally be a symmetric monoidal smooth category.

\subsection{Geometric bordism categories}

Let $\mathcal{G}$ be a geometry for $d$-dimensional manifolds, i.e., a stack on $\mathrm{Fam}^{d}$. In this section, we will define our symmetric monoidal smooth category (Definition 3.23) of $\mathcal{G}$-bordisms, denoted by $\mathcal{G}$ Bord. We start by defining a smooth symmetric monoidal stack $\mathcal{G}$ Bord $_{n}$ for every object $n \in \mathbb{Z}_{\geq 0}$. Afterwards, we define maps of symmetric monoidal stacks

$$
\kappa^{*}: \mathcal{G B o r d}_{n} \longrightarrow \mathcal{G} \operatorname{Bord}_{m}
$$

for every morphism $\kappa:[m] \rightarrow[n]$ in $\Delta$. These maps will satisfy $(\kappa \circ \eta)^{*}=\eta^{*} \circ \kappa^{*}$, so that we obtain a strict functor $\mathcal{G}$ Bord: $\Delta^{\mathrm{op}} \rightarrow \mathrm{St}^{\otimes}$. At the end of the subsection, we comment on the smooth category property of $\mathcal{G}$ Bord.

Definition 3.25 (the stack $\mathcal{G}$ Bord $_{n}$ ). An object of the stack $\mathcal{G}$ Bord $_{n}$ lying over a manifold $S$ consists of the following data:

(O1) A family of $d$-dimensional manifolds, that is, an object $X / S \in \mathrm{Fam}^{d}$.

(O2) A $\mathcal{G}$-structure on $X / S$, that is, an object $G_{X / S}$ of $\mathcal{G}(X / S)$.

(O3) Smooth functions $\rho_{a}: X \rightarrow \mathbb{R}$ for $a=0, \ldots, n$, subject to the following conditions:

(a) $\rho_{0} \geq \rho_{1} \geq \cdots \geq \rho_{n}$,

(b) whenever $\rho_{a}(x)=0, d \rho_{a}$ does not vanish on the vertical tangent space $T_{x}(X / S)$,

(c) the subspaces

$$
X_{a}^{b}:=\left\{x \in X \mid \rho_{a}(x) \geq 0 \geq \rho_{b}(x)\right\},
$$

are proper over $S$ for all $0 \leq a \leq b \leq n$. 


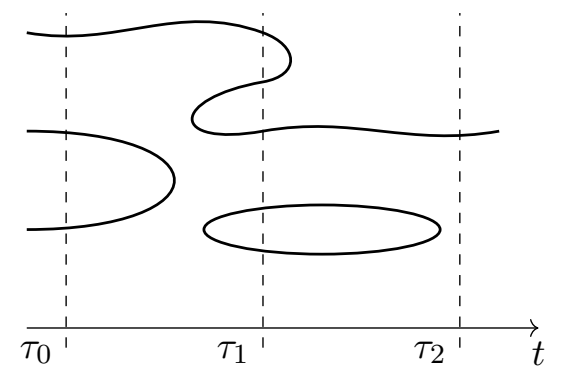

Figure 2. An object of 1-Bord 2 (pt), representing a pair of composable 1-dimensional bordisms. The cut functions are $\rho_{i}=t-\tau_{i}$.

We will abuse notation and abbreviate $X / S$ for objects, keeping in mind that the collection $\left\{\rho_{a}\right\}$ and the object $G_{X / S}$ are also part of the data. The subspace $X_{0}^{n}$ defined above will be called the core of $X / S$.

Morphisms of the stack $\mathcal{G} \operatorname{Bord}_{n}$ are going to be equivalence classes of maps, where a map

$$
\left(X / S ; \rho_{0}, \ldots, \rho_{n} ; G_{X / S}\right) \longrightarrow\left(Y / T ; \rho_{0}^{\prime}, \ldots, \rho_{n}^{\prime} ; G_{Y / T}\right)
$$

lying over a morphism $f: S \rightarrow T$ consists of the following data:

(M1) An open neighborhood $U$ of the core $X_{0}^{n}$ such that for some $\varepsilon>0$,

$$
U \supseteq \rho_{n}^{-1}(-\infty, \varepsilon) \cap \rho_{0}^{-1}(-\varepsilon, \infty)
$$

and

$$
F(U) \supseteq\left(\rho_{n}^{\prime}\right)^{-1}(-\infty, \varepsilon) \cap\left(\rho_{0}^{\prime}\right)^{-1}(-\varepsilon, \infty) .
$$

(M2) A smooth map $F: U \rightarrow Y$ covering $f$, which is a fiberwise open embedding onto a neighborhood of the core $Y_{0}^{n}$, such that for each $0 \leq a \leq n$, there exist positive smooth functions $\zeta_{a}$ on $U$ such that $F^{*} \rho_{a}^{\prime}=\left.\zeta_{a} \rho_{a}\right|_{U}$.

(M3) A morphism $\varphi:\left.\left.G_{X / S}\right|_{U / S} \rightarrow G_{Y / T}\right|_{F(U) / T}$ covering the morphism $(F, f): U / S \rightarrow F(U) / T$ in $\mathrm{Fam}^{d}$.

We declare two maps to be equivalent if they have a common restriction to a smaller neighborhood of the core. Here a restriction of a map $(f, F, \varphi)$ is a map of the form $\left(f,\left.F\right|_{V},\left.\varphi\right|_{V}\right)$ for some open neighborhood $V \subseteq U$ of the core satisfying (M1).

Remark 3.26. The condition relating $F^{*} \rho_{a}^{\prime}$ and $\left.\rho_{a}\right|_{U}$ in (M1) is equivalent to saying that these two functions have the same sign. We express it in terms of the positive function $\zeta_{a}$ so that our definition still makes sense, without change, in the supermanifold case.

Remark 3.27. To simplify the presentation, we implicitly choose a cleavage for the stack $\mathcal{G}$ (i.e., a preferred choice of pullback arrows) in order to define "restrictions" of objects in $\mathcal{G}$ such as $\left.G_{X / S}\right|_{U / S}$. Explicitly, this means a triangle $G_{X / S} \leftarrow G_{U / S} \rightarrow G_{Y / T}$ consisting of an object $G_{U / S}$ together with an arrow $G_{U / S} \rightarrow G_{X / S}$ covering the inclusion $U / S \hookrightarrow X / S$ and an arrow $G_{U / S} \rightarrow G_{Y / T}$ covering the map $(f, F): U / S \rightarrow Y / T$. These data are unique up to unique isomorphism.

Morphisms are composed as follows. Suppose that, in addition to the map $(f, F, \varphi)$ described above, we are given a second map $\left(f^{\prime}, F^{\prime}, \varphi^{\prime}\right)$ starting at $Y / T$. Choose a subset $V \subset U$ satisfying (M1) and such that $F(V) \subset U^{\prime}$ (where $U^{\prime}$ is the domain of $\left.F^{\prime}\right)$. Then $\left(f^{\prime} \circ f,\left.F^{\prime} \circ F\right|_{V}, \varphi^{\prime} \circ\right.$ 
$\left.\left.\varphi\right|_{V / S}\right)$ is a representative for the composition. It is straightforward to show that this operation on morphisms is independent of the choice of representatives.

Each of the stacks $\mathcal{G}$ Bord $_{n}$ is a symmetric monoidal stack with the tensor product given by fiberwise disjoint union, that is, $X / S \otimes Y / S:=(X \amalg Y) / S$. A $\mathcal{G}$-structure on $X / S \otimes Y / S$ is obtained by the stack property, using the obvious cover $\{X \rightarrow X \amalg Y, Y \rightarrow X \amalg Y\}$. Moreover, it is clear that the pullback maps $\kappa^{*}$ are monoidal, so that $\mathcal{G}$ Bord is a symmetric monoidal smooth category.

This concludes the construction of a category $\mathcal{G B o r d}_{n}$ with a projection onto Man, where an object $\left(X / S ; \rho_{0}, \ldots, \rho_{n} ; G_{X / S}\right)$ is mapped to $S$.

Remark 3.28. We think of an object in $\mathcal{G} \operatorname{Bord}_{n}(\mathrm{pt})$ as a sequence of $n$ composable bordisms, where the indiviual bordisms are the sets $X_{a-1}^{a}, a=1, \ldots, n$, defined in (3.6). These are $d$ manifolds with boundary by requirement $(b)$, unless we are in the degenerate case where $\rho_{a-1}$ and $\rho_{a}$ have common zeros. Objects in $\mathcal{G} \operatorname{Bord}_{n}(S)$ are thought of as families of such bordisms, parametrized by $S$.

Example 3.29. For $n=0$, we just have one cut function $\rho_{0}$, and the core $X_{0}^{0}=\rho_{0}^{-1}(0)$ is a codimension 1 submanifold of $X$, by condition $(\mathrm{O} 3)(b)$ above. This condition also implies that $X_{0}^{0}$ intersects the fibers of $X \rightarrow S$ transversally and hence determines a $(d-1)$-dimensional submanifold of each fiber $X_{s}$. Similarly, for $n=1$, the core $X_{0}^{1}$ determines, for each $s \in S$ such that $\rho_{0}(s)<\rho_{1}(s)$ everywhere, a $d$-manifold $X_{0}^{1} \cap X_{s}$ with boundary. It is compact, by condition $(\mathrm{O} 3)(c)$, thus a bordism between $X_{0}^{0} \cap X_{s}$ and $X_{1}^{1} \cap X_{s}$. In this way, $\mathcal{G}$ Bord $_{0}$ and $\mathcal{G}$ Bord $_{1}$ comprise families of bordisms and their boundaries. However, in the presence of a nontrivial geometry $\mathcal{G}$, there is the additional data of a neighborhood of the core together with a geometric structure on this neighborhood. This prohibits us to simply work directly with the cores.

Lemma 3.30. For each $n, \mathcal{G B o r d}_{n}$ is a stack.

Proof. We have to show that $\mathcal{G}$ Bord $_{n}$ is a prestack and that it satisfies descent.

First, we show that $\mathcal{G} \operatorname{Bord}_{n}$ is a fibered category. To this end, let $f: S^{\prime} \rightarrow S$ be a morphism, and let $B=\left(X / S ; \rho_{0}, \ldots, \rho_{n} ; G_{X / S}\right)$ be an object of $\mathcal{G} \operatorname{Bord}_{n}$ over $S$. Let $X^{\prime}=S^{\prime} \times_{S} X$ be the fiber product over $S$. This is an object of Fam over $S^{\prime}$, and we obtain a morphism $(f, F): X^{\prime} / S^{\prime} \rightarrow X / S$ in Fam. Set $\rho_{a}^{\prime}=\left(F^{\prime}\right)^{*} \rho_{a}$. Now $B^{\prime}=\left(X^{\prime} / S^{\prime} ; \rho_{0}^{\prime}, \ldots, \rho_{n}^{\prime} ; F^{*} G_{X^{\prime} / S^{\prime}}\right)$ is an object of $\mathcal{G} \operatorname{Bord}_{n}$ over $S^{\prime}$, and $(f, F, \varphi): B^{\prime} \rightarrow B$ is a morphism in $\mathcal{G} \operatorname{Bord}_{n}$ which is a cartesian lift of $f$.

To show that $\mathcal{G} \operatorname{Bord}_{n}$ is a prestack, by [15, Proposition 3.22], it now suffices to show that for any manifold $S, \mathcal{G} \operatorname{Bord}_{n}(S)$ is a groupoid. Let

$$
(\mathrm{id}, F, \varphi):\left(X / S ; \rho_{0}, \ldots, \rho_{n} ; G_{X / S}\right) \rightarrow\left(Y / S ; \rho_{0}^{\prime}, \ldots, \rho_{n}^{\prime} ; G_{Y / S}\right)
$$

be a map covering the identity, where $F$ is defined on some open neighborhood $U$ of the core $X_{0}^{n}$. Because $\left.F\right|_{U}$ is a fiberwise diffeomorphism onto its image covering the identity, it is in fact a diffeomorphism onto its image. Since $\mathcal{G}$ is a stack on Fam and $\varphi:\left.\left.G_{X / S}\right|_{U / S} \rightarrow G_{Y / S}\right|_{F(U) / S}$ covers the invertible morphism $\left(f,\left.F\right|_{U}\right)$, it is invertible. Hence $\left(\mathrm{id}, F^{-1}, \varphi^{-1}\right)$ is a morphism in the direct opposite of (id, $F, \varphi)$. It is clear that both compositions of these maps are restrictions of the identity map, hence equivalent to the identity. This shows that each morphism in $\mathcal{G} \operatorname{Bord}_{n}(S)$ is invertible, hence it is a groupoid.

To verify the descent property, let $S$ be a manifold and $\left\{S_{i}\right\}_{i \in I}$ a covering family of $S$. The objects of the category $\mathcal{G B o r d}_{n}\left(\left\{S_{i} \rightarrow S\right\}\right)$ of descent data are tuples $\left\{B_{i}\right\}_{i \in I}$ with $B_{i}=$ $\left(X_{i} / S_{i} ; \rho_{1}^{i}, \ldots, \rho_{n}^{i} ; G_{X_{i} / S_{i}}\right)$ and isomorphisms $F_{i j}:\left.\left.B_{i}\right|_{S_{i j}} \rightarrow B_{j}\right|_{S_{i j}}, i, j \in I$, where $S_{i j}=S_{i} \times_{S} S_{j}$. Morphisms in this category are tuples $\left\{H_{i}\right\}_{i \in I}$ of morphisms $H_{i}: B_{i} \rightarrow B_{i}^{\prime}$ in $\mathcal{G B o r d}_{n}$ that are compatible with the morphisms $F_{i j}$ in the sense that $\left.F_{i j} \circ H_{i}\right|_{S_{i j}}=\left.H_{j}\right|_{S_{i j}} \circ F_{i j}^{\prime}$. The functor 


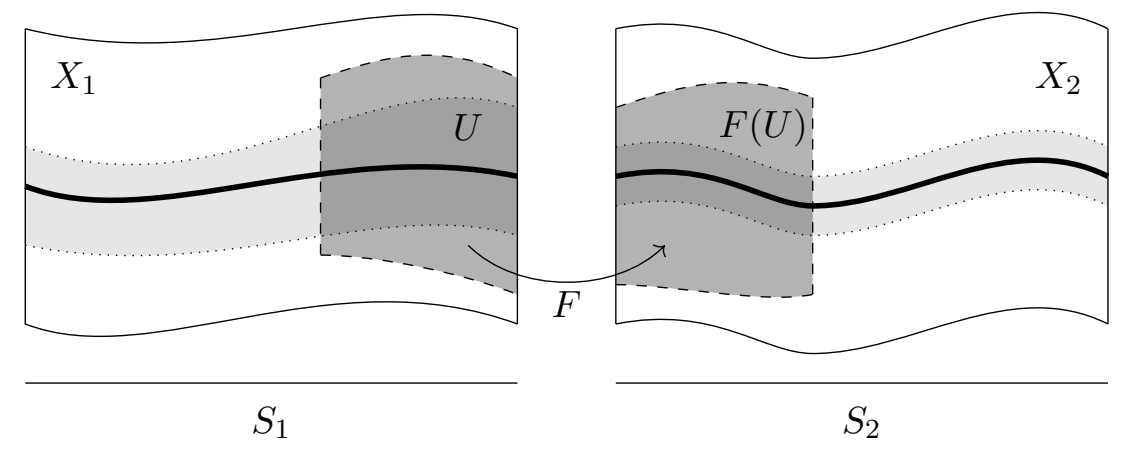

Figure 3. Picture of an object in $\mathcal{G} \operatorname{Bord}_{0}\left(\left\{S_{1}, S_{2} \rightarrow S\right\}\right)$. The light shaded regions depict the neighborhoods $\left(\rho_{0}^{i}\right)^{-1}(-\varepsilon, \varepsilon)$ of the core $\left(X_{i}\right)_{0}^{0}$ (drawn as a thick line). When attempting to glue, the difficulty is to shrink $X_{i}$ to $\tilde{X}_{i}$ in such a way that the corresponding restriction $\tilde{F}$ of $F$ maps $\left.\tilde{X}_{1}\right|_{S_{12}}$ diffeomorphically to $\left.\tilde{X}_{2}\right|_{S_{12}}$.

$\mathcal{G} \operatorname{Bord}_{n}(S) \rightarrow \mathcal{G} \operatorname{Bord}_{n}\left(\left\{S_{i} \rightarrow S\right\}\right)$ is given by restriction, i.e., by pullback along the inclusions $S_{i j} \rightarrow S_{i}$, respectively $S_{i} \rightarrow S$. It is clear that this functor is fully faithful, as morphisms are locally determined and glue.

To see that it is essentially surjective, let $\left\{B_{i}\right\}_{i \in I},\left\{F_{i j}\right\}_{i, j \in I}$ be an object as above. The maps $F_{i j}$ are defined on some open neighborhood $U_{i}$ of the core of $\left.X_{i}\right|_{S_{i j}}$ and come with morphisms $\varphi_{i j}:\left.\left.G_{X_{i} / S_{i}}\right|_{U_{i} / S_{i j}} \rightarrow G_{X_{j} / S_{j}}\right|_{U_{j} / S_{i j}}$ (where $U_{j}=F_{i j}\left(U_{i}\right)$ ). We need to show that the $B_{i}$ glue together to a bordism $B$ over $S$.

To begin with, we assume that the given cover of $S$ consists of two elements $S_{1}$ and $S_{2}$, with $S_{12}=S_{1} \times_{S} S_{2}$. Let $F: U / S_{12} \rightarrow F(U) / S_{12}$ be the gluing diffeomorphism. Let $\chi_{1}, \chi_{2}$ be a smooth partition of unity, i.e., non-negative functions with $\operatorname{supp}\left(\chi_{i}\right) \subset S_{i}$ and $\chi_{1}+\chi_{2}=1$. We denote the lifts of $\chi_{i}$ to $X_{1}$ and $X_{2}$ via the projections $X_{1} \rightarrow S_{1}$ and $X_{2} \rightarrow S_{2}$ by the same letter. Now for $a=0,1, \ldots, n$, consider the functions defined by

$$
\begin{aligned}
& \tilde{\rho}_{a}^{1}=\chi_{1} \cdot \rho_{a}^{1}+\chi_{2} \cdot F^{*} \rho_{a}^{2}, \\
& \tilde{\rho}_{a}^{2}=\chi_{1} \cdot\left(F^{-1}\right)^{*} \rho_{a}^{1}+\chi_{2} \cdot \rho_{a}^{2} .
\end{aligned}
$$

Let $\varepsilon>0$ be as in assumption (M3) on $F$ and set

$$
\tilde{X}_{i}=\left(\tilde{\rho}_{0}^{i}\right)^{-1}(-\infty, \varepsilon) \cap\left(\tilde{\rho}_{n}^{i}\right)^{-1}(-\varepsilon, \infty) \subseteq X_{i}, \quad i=1,2 .
$$

Let $\tilde{F}$ be the restriction of $F$ to $U_{1} \cap \tilde{X}_{1}$. Observe that by construction, $U_{1} \cap \tilde{X}_{1}=\left.\tilde{X}_{1}\right|_{S_{12}}$, and that $\tilde{F}$ maps $\left.\tilde{X}_{i}\right|_{S_{12}}$ diffeomorphically onto $\left.\tilde{X}_{j}\right|_{S_{12}}=F(U) \cap X_{2}$. Hence, $\tilde{X}_{1}$ and $\tilde{X}_{2}$ glue together, via $\tilde{F}_{12}$, to a family of manifolds over $S$. Since by construction, for each $a$, the functions $\rho_{a}^{1}$ and $\rho_{a}^{2}$ coincide over $S_{12}$, they combine to give functions $\rho_{0}, \ldots, \rho_{n}$ on $X$. Let $G_{\tilde{X}_{i} / S_{i}}=\left.G_{X_{i} / S_{i}}\right|_{\tilde{X}_{i} / S_{i}}$ be the restriction of the objects of $\mathcal{G}$ contained in the data of $B_{i}$ and let $\tilde{\varphi}$ be the corresponding restriction of the gluing isomorphism $\varphi$ in $\mathcal{G}$ convering $F$ (which then covers $\tilde{F})$. Since $\mathcal{G}$ satisfies descent, these objects $G_{\tilde{X}_{1} / S_{1}}, G_{\tilde{X}_{2} / S_{2}}$ glue together to an object $G_{X / S}$ over $X / S$. In total, we obtain an object $B=\left(X / S ; \rho_{0}, \ldots, \rho_{n} ; G_{X / S}\right)$ over $S$, together with maps $B \rightarrow B_{i}$ compatible with the gluing isomorphisms. In other words, we have found a preimage to the given object in $\mathcal{G B o r d}_{n}\left(\left\{S_{i} \rightarrow S\right\}\right)$.

Next, assume that the cover $\left\{S_{i}\right\}_{i \in I}$ is locally finite. Then $I$ must be countable, and we can identify $I=\mathbb{N}$. By replacing the functions $\rho_{a}^{i}$ by $\lambda_{i} \cdot \rho_{a}^{i}$ for suitable constants $\lambda_{i}>0$, we may achieve that the value $\varepsilon>0$ in the condition (M3) on the cut functions $F_{i j}$ can be chosen as $\varepsilon=1$ for all $i$ and $j$. This replaces $B_{i}$ by isomorphic objects in $\mathcal{G B o r d}_{n}$ and hence does not 
change the object in the category of descent data. Observe here that since the cover is locally finite, there are only finitely many non-trivial transition functions $F_{i j}$ for each fixed $i$, and hence only finitely many constraints for each $\lambda_{i}$. Hence the modification is indeed possible. Now, using the previous case, we may one by one glue together the corresponding bordisms $B_{1}, \ldots, B_{k}$ to a bordism $B^{(k)}$. Since the cover is locally finite, this may be achieved in such a way that over each compact subset of $S$, the bordisms $B^{(k)}$ are all canonically equivalent for $k$ large, hence they all glue together to a bordism $B$ over $S$.

For a general cover $\left\{S_{i}\right\}_{i \in I}$, we choose a locally finite subcover, which leads to an equivalent category of descent data.

Remark 3.31. The modification $\rho_{a}^{i} \mapsto \lambda_{i} \rho_{a}^{i}$ in the proof above was made to deal with the following technical problem: Without this change, the construction of $B^{(k+1)}$ might require a smaller choice of $\varepsilon>0$ than the one needed in the construction of $B^{(k)}$. Therefore, the total space $X^{(k+1)}$ of $B^{(k+1)}$ may be strictly smaller than that of $B^{(k)}$ and might degenerate in the limit $k \rightarrow \infty$.

The stacks $\mathcal{G} \operatorname{Bord}_{n}$ form a simplicial object in an obvious way: Given an order-preserving map $\kappa:[n] \rightarrow[m]$, we obtain a functor $\kappa^{*}: \mathcal{G} \operatorname{Bord}_{m} \rightarrow \mathcal{G} \operatorname{Bord}_{n}$, given by removing or duplicating the functions $\rho_{a}$. Forgetting a cut function has the interpretation of gluing bordisms along the boundary determined by it. Regarding the morphisms, we would like to say that they remain the same under $\kappa^{*}$. Precisely, what happens is the following. Notice that a map in $\mathcal{G} \operatorname{Bord}_{m}$ is also a map in $\mathcal{G} \operatorname{Bord}_{n}$. Hence, if a morphism in $\mathcal{G} \operatorname{Bord}_{m}$ is represented by a map $F$, we let $\kappa^{*}[F]$ be again the morphism represented by $F$, but with respect to the equivalence relation in $\mathcal{G} \operatorname{Bord}_{n}$. This makes sense because any two maps which are equivalent in $\mathcal{G}$ Bord $_{m}$ are also equivalent in $\mathcal{G} \operatorname{Bord}_{n}$, as follows directly from the definition.

Theorem 3.32. GBord is a symmetric monoidal smooth category.

Proof. To verify Definition 3.17(1), we have to show that the maps

$$
\mathcal{G B o r d}_{n}(S) \longrightarrow \mathcal{G B o r d}_{1}(S) \times{ }_{\mathcal{G} \operatorname{Bord}_{0}(S)} \cdots \times \times_{\mathcal{G} \operatorname{Bord}_{0}(S)} \mathcal{G} \operatorname{Bord}_{1}(S)
$$

are equivalences for each manifold $S$ and each $n \in \mathbb{N}$. This is clear, since gluing bordisms over a fixed parameter space is straightforward.

To verify Definition $3.17(2)$, we consider the categories $h_{0} \mathcal{G B o r d}(S)$ determined by the Segal set $n \mapsto h_{0} \mathcal{G} \operatorname{Bord}_{n}(S)$, for each manifold $S$. Objects $B$ of the stack $\mathcal{G B o r d}{ }_{1}(S)$ then determine morphisms $[B]$ in $h_{0} \mathcal{G B o r d}(S)$, and we have to analyze which objects give rise to invertible morphisms this way. We claim that for such a bordism $B=\left(X / S ; \rho_{0}, \rho_{1} ; G_{X / S}\right)$, the morphism $[B]$ is invertible if and only if $B$ is "thin", meaning that $\rho_{0}$ and $\rho_{1}$ have the same zero set. Given this claim, the completeness condition Definition 3.17(2) follows, since $B$ is isomorphic to $B^{\prime}=\left(X / S ; \rho_{0}, \rho_{0} ; G_{X / S}\right)$, which is in the image of the degeneracy map $\mathcal{G} \operatorname{Bord}_{0}(S) \rightarrow \mathcal{G} \operatorname{Bord}_{1}(S)$. To show the claim, assume that $[B]$ is invertible. Let $M=\left(X / S ; \rho_{0} ; G_{X / S}\right)$ be the left boundary of $B$, an object of $\mathcal{G} \operatorname{Bord}_{0}(S)$. Then there exists a bordism $B^{-1}$ such that $[B] \circ\left[B^{-1}\right]=\mathrm{id}_{[M]}$. The composition $[B] \circ\left[B^{-1}\right]$ can be represented by an element $B_{2} \in \mathcal{G} \operatorname{Bord}_{2}(S)$ with the property that $s_{1}^{*} B_{2}=B$ and $s_{2}^{*} B_{2}=B^{-1}$, where $s_{i}:[1] \rightarrow[n], i=1,2$, is the morphism sending $0 \mapsto i-1$ and $1 \mapsto i$. On the other hand, since $[B] \circ\left[B^{-1}\right]=\operatorname{id}_{[M]}$, the object $c^{*} B_{2}=\left(X / S ; \rho_{0}, \rho_{2} ; G_{X / S}\right)$ must be isomorphic, as an object of $\mathcal{G} \operatorname{Bord}_{1}(S)$, to the thin bordism $\operatorname{id}_{M}=\left(X / S ; \rho_{0}, \rho_{0} ; G_{X / S}\right)$. Here $c:[1] \rightarrow[2]$ maps $0 \rightarrow 0$ and $1 \rightarrow 2$. Therefore, $c^{*} B_{2}$ must be thin, i.e., $\rho_{0}$ and $\rho_{2}$ have the same zero sets. This implies that also $\rho_{1}$ must have the same zero set, which implies that both $B$ and $B^{-1}$ are thin as well. Conversely, if $B$ is thin, then, as mentioned above, it is isomorphic to $B^{\prime}=\left(X / S ; \rho_{0}, \rho_{0} ; G_{X / S}\right)$, which implies that $[B]=\mathrm{id}_{[M]}$. In particular, $[B]$ is invertible.

We conclude this section by giving some examples of geometric bordism categories. 
Example 3.33 ("no geometry"). If we choose $\mathcal{G}$ to be the trivial stack on Fam $^{d}$ (whose fibers are single points), we get the realization of the $d$-dimensional bordism category in the world of smooth categories, to be denoted $d$-Bord. Objects of $d$-Bord B $_{n}$ consist simply of a family $X / S$ of manifolds parametrized by $S$, together with cut functions $\rho_{0}, \ldots, \rho_{n}$. In this case, morphisms from $X / S$ to $Y / T$ are just given by smooth fiber-preserving maps $F$ that are defined on a neighborhood $U$ of the core, are fiberwise open embeddings and send $X_{a}^{b}$ to $Y_{a}^{b}$; two such maps $F, F^{\prime}$ (defined on $U, U^{\prime}$ ) are identified if they coincide on a smaller neighborhood $V \subset U \cap U^{\prime}$ of $X_{0}^{n}$.

Example 3.34 (bordisms over a manifold). If $\mathcal{G}$ is the $d$-dimensional geometry represented by a manifold $M$, as in Example 3.13, we get the category $d$-Bord $(M)$ of bordisms over $M$. Clearly, this specializes to the previous example if one takes $M$ to be a point. This is our main example in the second part of this paper (where moreover $d=1$ ).

Example 3.35 (orientations). If $\mathcal{G}$ is the $d$-dimensional geometry of fiberwise orientations, as in Example 3.14, we denote the resulting smooth category by $d$-Bord ${ }^{\text {or }}$. In this case, the fibers $X_{s}$ of an object $X / S \in d$-Bord ${ }_{n}^{\text {or }}$ carry orientations, and the maps $F$ are required to be orientationpreserving when restricted to the fibers. That this is a condition rather than additional data for $F$ reflects the fact that $\mathcal{G}$ is a sheaf on $\mathrm{Fam}^{d}$, i.e., discrete as a stack.

\subsection{Geometric field theories}

Conceptually, a field theory should be a symmetric monoidal functor from a suitable bordism category to the category of vector spaces. To put this concept into our setup, we need our source and target categories, as well as the functor, to be smooth. As source we take $\mathcal{G}$ Bord for some geometry $\mathcal{G}$, as defined above. To specify the target, we need to fix a notion of "smooth family" of vector spaces. Initially, we will study field theories taking values in the smooth category Vect of finite-dimensional vector bundles on Man, obtained by applying the procedure of Example 3.19 to the stack (of categories) of finite-dimensional vector bundles. Later, in Section 5, we will consider the more general case of $C^{\infty}$-modules. These categories are symmetric monoidal using the tensor product of vector spaces, respectively modules.

Definition 3.36 (geometric field theories). Let $\mathcal{G}$ be a $d$-dimensional geometry. A ( $d$-dimensional) field theory with geometry $\mathcal{G}$ is a symmetric monoidal smooth functor

$Z: \mathcal{G B o r d} \longrightarrow$ Vect.

A morphism of field theories is a smooth, symmetric monoidal natural transformation.

Denoting by Fun ${ }^{\otimes}$ the groupoid of functors between smooth categories, together with smooth, invertible natural transformations, we denote by

$$
\mathcal{G} \mathrm{FT}:=\operatorname{Fun}^{\otimes}(\mathcal{G B o r d}, \text { Vect })
$$

the groupoid of functorial field theories for a given geometry $\mathcal{G}$.

Field theories as functors between smooth categories are complicated objects, due to the fact that stacks form a 2-category. A field theory $Z$ consists of the following data. First, for every object $[n] \in \Delta$, there is a map of stacks

$$
Z_{n}: \mathcal{G B o r d}_{n} \longrightarrow \operatorname{Vect}_{n}
$$

However, since stacks form a 2-category, we cannot expect that these strictly commute with the structure maps in $\Delta$; instead, for each morphism $\kappa:[m] \rightarrow[n]$ in $\Delta$, there is a 2-morphism 
(i.e., a natural isomorphism) $\zeta^{\kappa}: Z_{m} \kappa^{*} \rightarrow \kappa^{*} Z_{n}$. If $\eta:[k] \rightarrow[m]$ is another map in $\Delta$, then the corresponding 2-morphisms have to satisfy the coherence condition

$$
\zeta^{\kappa \circ \eta}=\eta^{*} \zeta^{\kappa} \circ \zeta^{\eta} \kappa^{*}
$$

Visually, this is depicted by

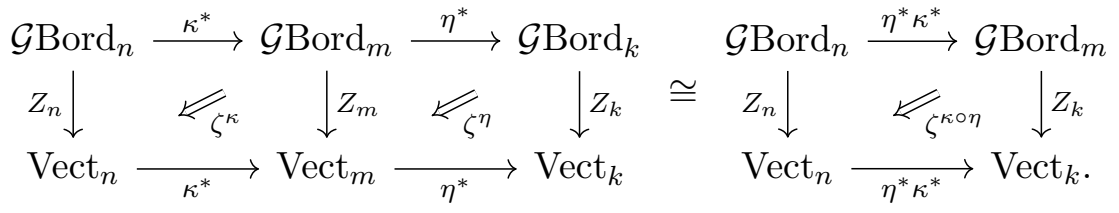

(Here, we are using the strictness of $\mathcal{G B o r d}$ and Vect, that is, the fact that $(\kappa \eta)^{*}$ and $\eta^{*} \kappa^{*}$ are equal; otherwise the identification of the two diagrams would involve, additionally, the coherence data $\eta^{*} \kappa^{*} \cong(\kappa \eta)^{*}$.) Fortunately, in our setting, the data of a field theory can be simplified considerably, as the following lemma shows.

Lemma 3.37 (strictification). Let $\mathcal{B}$ be a strict smooth category and let $\mathcal{V}$ be a smooth category obtained from the procedure of Example 3.19. Then any smooth functor $Z: \mathcal{B} \rightarrow \mathcal{V}$ has a canonical strictification, that is, there exists a canonically isomorphic functor $Z^{\prime}=\left\{Z_{n}^{\prime}, \zeta^{\prime \kappa}\right\}$ such that $Z_{m}^{\prime} \kappa^{*}=\kappa^{*} Z_{n}^{\prime}$ and $\zeta^{\prime \kappa}=\mathrm{id}$ for every morphism $\kappa:[m] \rightarrow[n]$ in $\Delta$.

Proof. Fix an integer $n \geq 0$ and $X \in \mathcal{B}_{n}$, and denote by $\kappa_{i}:[0] \rightarrow[n], 0 \leq i \leq n$, the morphism in $\Delta$ mapping $0 \mapsto i$. Set

$$
V_{i}=Z_{n}\left(\kappa_{i}^{*} X\right) \quad \text { and } \quad W_{i}=\kappa_{i}^{*}\left(Z_{n}(X)\right),
$$

and note that we have natural isomorphisms

$$
\zeta^{i}=\zeta_{X}^{\kappa_{i}}: V_{i} \rightarrow W_{i}
$$

By our assumption on $\mathcal{V}, Z_{n}(X) \in \mathcal{V}_{n}$ is a chain of morphisms

$$
Z_{n}(X)=\left(W_{0} \stackrel{\alpha_{1}}{\longrightarrow} W_{1} \stackrel{\alpha_{2}}{\longrightarrow} \cdots \stackrel{\alpha_{n}}{\longrightarrow} W_{n}\right)
$$

in the stack of which $\mathcal{V}$ is the nerve. Towards defining the functor $Z_{n}^{\prime}: \mathcal{B}_{n} \rightarrow \mathcal{V}_{n}$, set $Z_{n}^{\prime}(X)$ to be chain of morphisms $V_{0} \rightarrow \cdots \rightarrow V_{n}$ such the diagram below commutes:

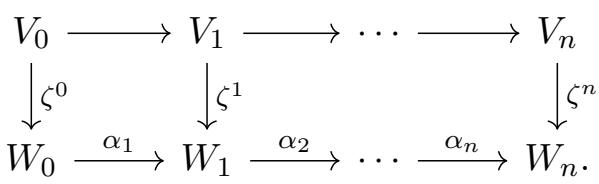

The above diagram also fixes the effect of $Z_{n}^{\prime}$ on morphisms of $\mathcal{B}_{n}$, if we insist that the collection $\left(\zeta^{0}, \ldots, \zeta^{n}\right)$ defines a natural transformation $\xi_{n}: Z_{n}^{\prime} \rightarrow Z_{n}$.

It remains to show that the collection $\left\{Z_{n}^{\prime}\right\}$ defines a strict smooth functor, that is, the diagram

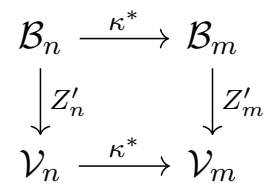


commutes strictly for every morphism $\kappa:[m] \rightarrow[n]$ in $\Delta$. Now, $\kappa^{*} Z_{n}^{\prime}(X)$ is the chain of morphisms

$$
V_{\kappa(0)} \rightarrow V_{\kappa(1)} \rightarrow \cdots \rightarrow V_{\kappa(m)},
$$

obtained from $Z_{n}^{\prime}(X)$ by appropriate compositions or insertion of identities. On the other hand, $Z_{m}^{\prime}\left(\kappa^{*} X\right)$ is a chain of morphisms of the form

$$
Z_{0}\left(\kappa_{0}^{*}\left(\kappa^{*} X\right)\right) \rightarrow Z_{0}\left(\kappa_{1}^{*}\left(\kappa^{*} X\right)\right) \rightarrow \cdots \rightarrow Z_{0}\left(\kappa_{m}^{*}\left(\kappa^{*} X\right)\right) .
$$

By strictness of $\mathcal{B}$ as a simplicial object, we have $\kappa_{i}^{*} \kappa^{*} X=\left(\kappa \circ \kappa_{i}\right)^{*} X=\kappa_{\kappa(i)}^{*} X$, so that the $i$ th object in (3.9) is $Z_{0}\left(\kappa_{i}^{*}\left(\kappa^{*} X\right)\right)=V_{\kappa(i)}$. It remains to see that the morphisms in the chains (3.8) and (3.9) are identical. Consider the commutative diagram below:

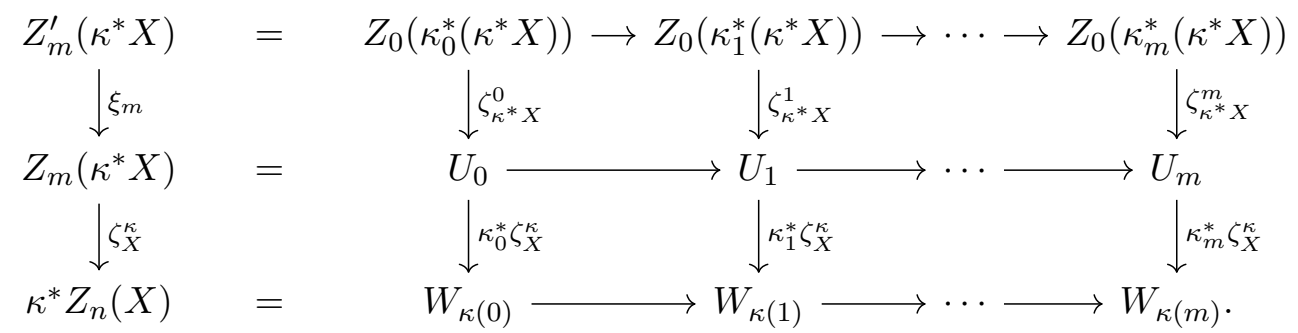

We will be done if we show that the $i$ th composite vertical map is equal to $\zeta_{X}^{\kappa(i)}: V_{\kappa(i)} \rightarrow W_{\kappa(i)}$, since in this case we can replace the top row by (3.8) and still have a commutative diagram. But this fact is simply the coherence condition (3.7), applied to the case $\eta=\kappa_{i}$.

Remark 3.38 (field theories as strict functors). Since our bordism categories, as well as the smooth category of vector bundles, satisfy the assumptions of the above lemma, it follows that we make no mistake by defining field theories as strict symmetric monoidal functors $Z: \mathcal{G B o r d} \rightarrow$ Vect, and their morphisms as strict natural transformations. We will work in this context in the next section, which simplifies our life considerably.

\section{Classification of one-dimensional field theories}

In this section, we discuss the classification of one-dimensional field theories over a manifold $M$. Let us briefly discuss the classical case (with ordinary categories and $M=\mathrm{pt}$ ) in order to see what to expect. The one-dimensional (ordinary) bordism category 1-Bord is easy to describe. The objects, compact zero-dimensional manifolds, are just finite collections of points. To understand the morphisms, one needs the classification of compact, connected one-dimensional manifolds with boundary; this classification is very simple (say, using Morse theory). Apart from the circle, which is the only closed example, we have two elbows (the one with two incoming boundary components and zero outcoming boundary components, as well as its dual) and the interval (with one incoming and one outgoing boundary component).

We briefly recall the well-known construction of one-dimensional field theories from vector spaces.

Construction 4.1 (unoriented 1-TFTs). In the unoriented case, a field theory can be obtained from the data of a finite-dimensional vector space $V$ over $\mathbb{K}=\mathbb{R}$ or $\mathbb{C}$ together with a symmetric nondegenerate bilinear form $\beta$ as follows:

1. To a collection of $k$ points, we assign the $k$-fold tensor product $V^{\otimes k}$. In particular, the ground field $\mathbb{K}$ corresponds to the empty set. 


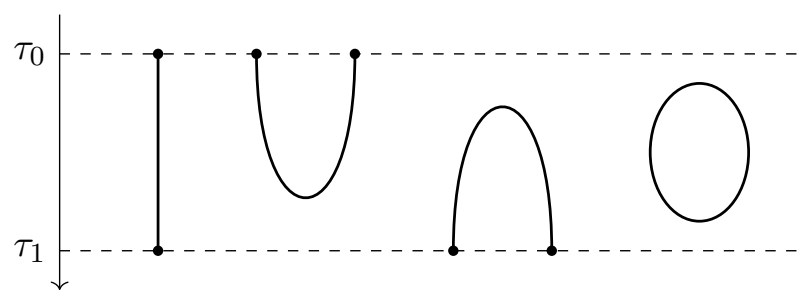

Figure 4. All possible connected unoriented one-dimensional bordisms. We call them interval, left elbow, right elbow, and circle, respectively. The cut functions are $\rho_{i}=t-\tau_{i}$, so these pictures are read from top to bottom.

2. To the interval, we assign the identity homomorphism on $V$.

3. To the elbow with two incoming boundary components, we assign the bilinear form $\beta$.

4. To the elbow with two outgoing boundary components, we assign $\tau:=\sum_{i=1}^{n} \varepsilon_{i} b_{i} \otimes b_{i} \in V \otimes V$, where $b_{1}, \ldots, b_{n}$ is a generalized orthonormal basis for $\beta$. This means that $\beta\left(b_{i}, b_{j}\right)=\varepsilon_{i} \delta_{i j}$, where $\varepsilon= \pm 1$ depending on the signature of $\beta$.

5. To the circle, we assign the number $n=\operatorname{dim}(V)$.

There are several things to check in order to see that this defines a field theory: For example, one has to check that $\beta \circ \tau=n$, as well as the snake identity

$$
(\beta \otimes \mathrm{id}) \circ(\mathrm{id} \otimes \tau)=\mathrm{id} .
$$

Conversely, any one-dimensional field theory $Z$ determines such a pair $(V, \beta)$ : just set $V=Z$ (pt) and $\beta$ to be the value of the elbow with two incoming boundary components. Then it follows from the snake identity (4.1) that $V$ must be finite-dimensional and $\beta$ must be nondegenerate. Moreover, the fact that $\beta$ must be symmetric follows from the observation that the elbows have an automorphism that switches the two boundary components.

The above construction can be upgraded to an equivalence of categories

$$
1-\mathrm{TFT} \cong \operatorname{Vect}_{\beta} \tilde{}
$$

where $\operatorname{Vect}_{\beta} \tilde{\beta}$ is the groupoid of finite-dimensional vector spaces equipped with a nondegenerate symmetric bilinear form, with maps being isometries.

Things change if we equip our bordisms with non-discrete data. In the following, we will consider the geometry where objects $X / S$ come equipped with a smooth map $\gamma: X \rightarrow M$, where $M$ is some fixed target manifold, as in Example 3.34. As a first approximation, we can think of objects of the bordism category as points in $M$, while morphisms are essentially paths in $M$. The corresponding smooth category is denoted by 1-Bord $(M)$, and the category of field theories will be denoted by

$$
1-\operatorname{TFT}(M):=\operatorname{Fun}^{\otimes}(1-\operatorname{Bord}(M), \operatorname{Vect}) .
$$

It turns out that this groupoid is equivalent to the groupoid $\operatorname{Vect} \widetilde{\nabla}, \beta(M)$, the objects of which are finite-dimensional vector bundles over $M$ with a fiberwise nondegenerate symmetric bilinear form and a compatible connection, and the morphisms of which are connection-preserving isometries.

Theorem 4.2 (classification of 1-TFTs). There is an equivalence of categories

$$
1-\operatorname{TFT}(M) \cong \operatorname{Vect}_{\nabla, \beta}^{\sim}(M)
$$

which is natural in $M$. 
The remainder of this section is dedicated to the proof of this result. First, in Section 4.1, we explain how to construct elements of $1-\operatorname{TFT}(M)$ from a vector bundle with connection and bilinear form, in a functorial way, cf. Proposition 4.4 below. While a little tricky in the detail, this is more or less the standard construction. The main work of the proof is done in Section 4.2, where we restrict our attention to the path subcategory of the bordism category, where all issues already arise. First, we restrict to the case that paths have sitting instants near the marked points (see Definition 4.8 for the precise definition), which is rather standard. The main new idea is then to reduce the general case to this one using so-called modification functions. The proof is then finished in Section 4.4. Finally, in Section 4.5, we comment on the oriented case.

In order to prove Theorem 4.2, one needs a result that reconstructs a connection from parallel transport data. To set up the one we use, denote by $C^{\infty}([0,1], M)$ the set of smooth maps from $[0,1]$ to $M$. It has a natural (infinite-dimensional) smooth manifold structure modelled on a nuclear Fréchet space, and there are smooth evaluation maps

$$
\mathrm{ev}_{t}: \quad C^{\infty}([0,1], M) \rightarrow M, \quad \gamma \mapsto \gamma(t)
$$

for $t \in[0,1]$. Thus, given a vector bundle $V$ over $M$, we can form the pullback bundles $\mathrm{ev}_{t}^{*} V$. The tensor product $\mathrm{ev}_{0}^{*} V^{\vee} \otimes \mathrm{ev}_{1}^{*} V$ is the vector bundle over $C^{\infty}([0,1], M)$ whose fiber at a path $\gamma$ is given by $\operatorname{Hom}\left(V_{\gamma(0)}, V_{\gamma(1)}\right)$. Finally, given $0 \leq a \leq b \leq 1$, we let $\mathfrak{s}_{a, b}$ be the smooth map from $C^{\infty}([0,1], M)$ to itself defined by

$$
\left(\mathfrak{s}_{a, b} \gamma\right)(t)=\gamma(a+(b-a) t) .
$$

Proposition 4.3. Let $V$ be a vector bundle over $X$ and let $P$ be a smooth section of the bundle $\mathrm{ev}_{0}^{*} V^{\vee} \otimes \mathrm{ev}_{1}^{*} V$ over $C^{\infty}([0,1], X)$. Assume that $P$ maps constant paths to the identity and that we have

$$
P\left(\mathfrak{s}_{a, 1} \gamma\right) \circ P\left(\mathfrak{s}_{0, a} \gamma\right)=P(\gamma)
$$

for all $\gamma \in C^{\infty}([0,1], M)$, where $\mathfrak{s}_{a, b}$ is the cutting-and-rescaling map defined above. Then there exists a unique connection $\nabla$ on $V$ such that $P$ is the parallel transport along $\nabla$.

Similar results were obtained by Freed [4, Proposition B1] and Schreiber and Waldorf [10, Lemma 4.1]; cf. also [2, Lemma 4.9].

Proof. For $v \in T_{p} M$, let $\gamma \in C^{\infty}([0,1], M)$ be a path such that $\gamma(t)=p$ and $\dot{\gamma}(t)=v$ for some $t \in(0,1]$. For any section $u$ of $V$, set

$$
\nabla_{v} u(p):=-\left.\frac{\mathrm{d}}{\mathrm{d} \varepsilon}\right|_{\varepsilon=0} P\left(\mathfrak{s}_{t-\varepsilon, t} \gamma\right) u(\gamma(t-\varepsilon)),
$$

noting that $P\left(\mathfrak{s}_{t-\varepsilon, 1} \gamma\right) u(\gamma(t-\varepsilon)) \in V_{p}$ for each $\varepsilon$, hence differentiation makes sense. We proceed to show that this definition is independent of the choice of $\gamma$ and $t$ and defines a connection on $V$. In fact, in a local trivialization of the bundle $V$, we have

$$
\begin{aligned}
\left(\nabla_{v} u\right)^{i}(p) & =-\left.\frac{\mathrm{d}}{\mathrm{d} \varepsilon}\right|_{\varepsilon=0} P_{j}^{i}\left(\mathfrak{s}_{t-\varepsilon, t} \gamma\right) u^{j}(\gamma(t-\varepsilon)) \\
& =-\left.P_{j}^{i}\left(\mathfrak{s}_{t, t} \gamma\right) \frac{\mathrm{d}}{\mathrm{d} \varepsilon}\right|_{\varepsilon=0} u^{j}(\gamma(t-\varepsilon))-\left(\left.\frac{\mathrm{d}}{\mathrm{d} \varepsilon}\right|_{\varepsilon=0} P_{j}^{i}\left(\mathfrak{s}_{1-\varepsilon, 1} \gamma\right)\right) u^{j}(p) \\
& =\left(\partial_{v} u^{i}\right)(p)-\left.u^{j}(p) \mathrm{dP}_{j}^{i}\left(\mathfrak{s}_{t, t} \gamma\right) \frac{\mathrm{d}}{\mathrm{d} \varepsilon}\right|_{\varepsilon=0} ^{\mathfrak{s}_{t-\varepsilon, t} \gamma},
\end{aligned}
$$


where we used that $\left(\mathfrak{s}_{t, t} \gamma\right)(t) \equiv p$ and $P_{j}^{i}\left(\mathfrak{s}_{t, t} \gamma\right)=\delta_{j}^{i}$, since $P$ maps constant paths to the identity. Now notice the vector field $\left.\frac{\mathrm{d}}{\mathrm{d} \varepsilon}\right|_{\varepsilon=0} \mathfrak{s}_{t-\varepsilon, t} \gamma$ along the constant path $\mathfrak{s}_{t, t} \gamma$ is given by

$$
\left(\left.\frac{\mathrm{d}}{\mathrm{d} \varepsilon}\right|_{\varepsilon=0} \mathfrak{s}_{t-\varepsilon, t} \gamma\right)(s)=\left.\frac{\mathrm{d}}{\mathrm{d} \varepsilon}\right|_{\varepsilon=0}\left(\mathfrak{s}_{t-\varepsilon, t} \gamma\right)(s)=\left.\frac{\mathrm{d}}{\mathrm{d} \varepsilon}\right|_{\varepsilon=0} \gamma(t-\varepsilon+\varepsilon s)=s \dot{\gamma}(t)=s v .
$$

Hence $\nabla_{v} u(p)$ is independent of the choice of $\gamma$. Define

$$
\omega_{j}^{i}(p) v:=-\mathrm{d} P_{j}^{i}(p) V
$$

where $p$ denotes the path constant equal to $p$ and $V$ denotes the $T_{p} M$-valued function $V(t)=t v$. Observe that $\omega_{j}^{i}$ defines a matrix of one-forms on $T_{p} M$. Then

$$
\left(\nabla_{v} u\right)^{i}(p)=\partial_{v} u^{i}(p)+u^{j}(p) \omega_{j}^{i}(p) v,
$$

hence $\nabla_{v}$ is a connection with Christoffel symbols $\omega_{j}^{i}$. Finally, fix $\gamma \in C^{\infty}([0,1], X)$ and $u_{0} \in \gamma(0)$ and let $u(t):=P\left(\mathfrak{s}_{0, t} \gamma\right) u_{0}$. Then we have $u(0)=u_{0}$ and using $\left(\mathfrak{s}_{t-\varepsilon, t} \gamma\right)^{\cdot}(t)=t \dot{\gamma}(t)$, we obtain

$$
\begin{aligned}
\frac{\nabla}{\mathrm{d} t} u(t) & =-\left.\frac{\mathrm{d}}{\mathrm{d} \varepsilon}\right|_{\varepsilon=0} P\left(\mathfrak{s}_{t-\varepsilon, t} \gamma\right) u(t-\varepsilon) \\
& =-\left.\frac{\mathrm{d}}{\mathrm{d} \varepsilon}\right|_{\varepsilon=0} P\left(\mathfrak{s}_{t-\varepsilon, t} \gamma\right) P\left(\mathfrak{s}_{0, t-\varepsilon}\right) u_{0} \\
& =-\left.\frac{\mathrm{d}}{\mathrm{d} \varepsilon}\right|_{\varepsilon=0} P\left(\mathfrak{s}_{0, t} \gamma\right) u_{0}=0 .
\end{aligned}
$$

Hence $u(t)$ is the parallel transport of $u_{0}$ along $\gamma$.

\subsection{Construction of field theories from vector bundles}

Let $M$ be some fixed target manifold. In this section, we construct a field theory from the data of an object $(V, \nabla, \beta) \in \operatorname{Vect}_{\nabla, \beta}(M)$. More precisely, we prove the following proposition.

Proposition 4.4 (construction of field theories). There is a functor

$\Phi: \quad \operatorname{Vect}_{\nabla, \beta}(M) \longrightarrow 1-\operatorname{TFT}(M)$,

which is fully faithful and natural in $M$.

Breaking down our general definition to this special case, an object of 1-Bord $(M)_{n}$ lying over a manifold $S$ is given by a family $X / S$ of one-dimensional manifolds $X_{s}, s \in S$, together with a map $\gamma: X \rightarrow M$ and functions $\rho_{0}, \ldots, \rho_{n}: X \rightarrow \mathbb{R}$ which cut out codimension-one submanifolds

$$
X_{a}^{a}=\left\{x \in X \mid \rho_{a}(x)=0\right\} .
$$

The properness assumption (O3c) implies that the restrictions $X_{a}^{a} \cap X_{s}$ to the fibers are compact, i.e., finite collections of points. More generally, we have the following result.

Lemma 4.5. The submanifold $X_{a}^{a}$ of $X$ is a finite covering of $S$ (with possibly empty fibers).

Proof. Let $s \in S$ and $x \in X_{a}^{a} \cap X_{s}$, and denote by $\pi: X \rightarrow S$ the projection. Since $\left.\mathrm{d} \rho_{a}\right|_{X_{s}}(x)$ $\neq 0, X_{a}^{a}$ intersects the fiber $X_{s}$ transversally, that is, $\mathrm{d} \pi(x)$ is an isomorphism when restricted to the tangent space $T_{x} X_{a}^{a}$. Therefore, $\left.\pi\right|_{X_{a}^{a}}$ is a local diffeomorphism. Furthermore, by assumption, $X_{a}^{a}$ is proper over $S$, meaning that $\left.\pi\right|_{X_{a}^{a}}$ is a proper map. However, a proper local diffeomorphism is a covering map the fibers of which have at most finitely many points (possibly zero). 
To prove Proposition 4.4, we start by constructing a field theory from the data of a vector bundle with non-degenerate bilinear form and compatible connection.

Let $(V, \nabla, \beta) \in \operatorname{Vect}_{\nabla, \beta}(M)$. Our goal is to construct a field theory $Z_{V, \nabla, \beta}$, which will be the value of $(V, \nabla, \beta)$ under the functor $\Phi$ in Proposition 4.4. Recall that the smooth functor $Z_{V, \nabla, \beta}$, as a morphism of simplicial objects, will consist of a sequence of stack maps 1-Bord $(X)_{n} \rightarrow$ Vect $_{n}, n \in \Delta$. At the $n$th simplicial level, we must have

$$
Z_{V, \nabla, \beta}\left(X / S ; \rho_{0}, \ldots, \rho_{n} ; \gamma\right)=\left(W_{0}, \ldots, W_{n} ; f_{1}, \ldots, f_{n}\right)
$$

for some vector bundles $W_{a}$ over $S$ and vector bundle maps $f_{a}: W_{a-1} \rightarrow W_{a}$. To define $W_{a}$, for each $a=0, \ldots, n$, set first $\tilde{W}_{a}:=\left(\left.\gamma\right|_{X_{a}^{a}}\right)^{*} V$, which makes sense since, for each $a=0, \ldots, n$, $X_{a}^{a}$ is a codimension-one submanifold of $X$. By Lemma 4.5, $X_{a}^{a}$ is a finite covering of $S$, hence any small enough open $U \subset S$ is covered by $U_{1}, \ldots, U_{k} \subset X_{a}^{a}$ such that the projection map $\pi$ provides diffeomorphisms $\left.\pi\right|_{U_{j}}: U_{j} \rightarrow U$. Hence we can set

$$
\left.W_{a}\right|_{U}:=\left(\left.\pi\right|_{U_{1}} ^{-1}\right)^{*} \tilde{W}_{a} \otimes \cdots \otimes\left(\left.\pi\right|_{U_{k}} ^{-1}\right)^{*} \tilde{W}_{a}
$$

where $\left.W_{a}\right|_{U}=\mathbb{C}$ if $k=0$. These vector bundles glue together to a vector bundle $W_{a}$ over $S$.

To define $f_{a}$ for each $a=1, \ldots, n$, consider the subsets $X_{a-1}^{a}$. Let $Y^{1}, \ldots, Y^{k}$ be the connected components of $\left.X_{a-1}^{a}\right|_{U}$, where $U \subset S$ is a small connected open as above for both $a-1$ and $a$. The map $\left.f_{a}\right|_{U}$ will be the tensor product of maps $f_{a}^{j}$, where $f_{a}^{j}$ is determined by the connected component $Y^{j}$. Each $f^{j}$ will be a vector bundle map

$$
f_{a}^{j}: \bigotimes_{Z}\left(\left.\pi\right|_{Z} ^{-1}\right)^{*} \tilde{W}_{a-1} \longrightarrow \bigotimes_{Z^{\prime}}\left(\left.\pi\right|_{Z^{\prime}} ^{-1}\right)^{*} \tilde{W}_{a-1}
$$

where $Z$ runs over the connected components of $Y^{j} \cap X_{a-1}^{a-1}$ and $Z^{\prime}$ runs over the connected components of $Y^{j} \cap X_{a}^{a}$ (by possibly making $U$ smaller, we can assume that the projection map is a diffeomorphism to $U$ when restricted to any one of these sets $Z$ and $Z^{\prime}$ ). The tensor product $\left.f_{a}\right|_{U}:=f_{a}^{1} \otimes \cdots \otimes f_{a}^{k}$ is then indeed a vector bundle map $\left.\left.W_{a-1}\right|_{U} \rightarrow W_{a}\right|_{U}$, by definition (4.3).

Now, each $Y^{j}$ is, essentially, either a circle bundle or an interval bundle over $U$; this only fails to be the case if $\rho_{a-1}(x)=\rho_{a}(x)$ at some points $\left.x \in X_{a-1}^{a}\right|_{U}$. To address this issue, we let

$$
U_{\circ}^{j}:=\left\{s \in U \mid \rho_{a-1}(y) \neq \rho_{a}(y) \text { for each } y \in Y^{j} \cap X_{s}\right\},
$$

which is an open subset of $U$. The complement $U \backslash U_{\circ}^{j}$ is the set where $Y^{j}$ is a "thin bordism", in the sense that

$$
\left.Y^{j}\right|_{s}=\left.\left(X_{a-1}^{a-1} \cap Y^{j}\right)\right|_{s}=\left.\left(X_{a}^{a} \cap Y^{j}\right)\right|_{s} \quad \text { for } \quad s \in U \backslash U_{\circ}^{j}
$$

Note in particular that for $s \in U \backslash U_{\circ}^{j},\left.Y^{j}\right|_{s}$ consists of finitely many points.

Lemma 4.6. For each $j=1, \ldots, k$, write $Y_{\circ}^{j}:=\left.Y_{j}\right|_{U_{\circ}^{j}}$. Then $Y_{\circ}^{j} \rightarrow U_{\circ}^{j}$ is a fiber bundle whose fibers are compact one-dimensional manifolds with boundary (thus, either intervals or circles). Moreover, if $Y_{\circ}^{j}$ is a circle bundle, then $U_{\circ}^{j}=U$ and $Y_{\circ}^{j}=Y^{j}$.

Proof. By construction, the total space $Y_{\circ}^{j}$ is a compact manifold and the projection $\left.\pi\right|_{Y_{0}^{j}}$ : $Y_{\circ}^{j} \rightarrow U_{\circ}^{j}$ is a proper submersion, hence a fiber bundle (with possibly empty fibers). The last statement follows from the fact that circle bundles cannot degenerate, by the requirements on the functions $\rho_{a}$. 
By possibly shrinking $U$ further, we may assume moreover that all these bundles are trivial. We now define $f^{j}$ case by case.

Suppose first that $Y_{0}^{j}$ is a circle bundle, so that $Y_{0}^{j}=Y^{j}$ and $U_{0}^{j}=U$. In this case, $Y^{j} \cap X_{a-1}^{a-1}=Y^{j} \cap X_{a}^{a}=\varnothing$, hence we have to produce a vector bundle map from the trivial line bundle to itself, that is, a function on $U$. Choose a trivialization $\varphi: U \times S^{1} \rightarrow Y^{j}$. Now set

$$
f^{j}:=\operatorname{tr} P(\varphi)
$$

where $P(\varphi)$ is the parallel transport around the loops $\varphi_{s}: S^{1} \rightarrow Y^{j} \subset X$ given by $\varphi_{s}(t)=\varphi(s, t)$ with respect to the pullback connection of $\gamma^{*} V \rightarrow X$. We claim that $f^{j}$ is independent of the choice of $\varphi$. If $\tilde{\varphi}$ is another trivialization of $Y^{j}$ that induces the same orientation on the fibers and agrees with $\varphi$ at the basepoint $1 \in S^{1}$, then it is just a reparametrization of $\varphi$, and our claim follows from the invariance of parallel transport under reparametrizations. Without the assumption on basepoints, $P\left(\varphi_{s}\right)$ and $P\left(\tilde{\varphi}_{s}\right)$ are conjugates for each $s \in U$, so the trace $f^{j}$ is still independent of the choice of $\varphi$. Finally, if $\tilde{\varphi}$ induces the opposite orientation, then $P\left(\tilde{\varphi}_{s}\right)=P\left(\varphi_{s}\right)^{-1}$. This yields the same trace, since $P(\varphi)$ preserves the bilinear form $\beta$; the calculation is

$$
\operatorname{tr} P\left(\varphi_{s}\right)=\sum_{i=1}^{n} \varepsilon_{i} \beta\left(P\left(\varphi_{s}\right) b_{i}, b_{i}\right)=\sum_{i=1}^{n} \varepsilon_{i} \beta\left(b_{i}, P\left(\varphi_{s}\right) b_{i}\right)=\sum_{i=1}^{n} \varepsilon_{i} \beta\left(P\left(\varphi_{s}\right)^{-1} b_{i}, b_{i}\right)=\operatorname{tr} P\left(\tilde{\varphi}_{s}\right),
$$

where $b_{1}, \ldots, b_{n}$ is a generalized orthonormal basis for $\beta$ and we used the symmetry of $\beta$ (note that $\beta$ is not assumed to be Hermitian in the complex case).

Suppose now that $Y_{\circ}^{j}$ is a bundle of intervals. In this case, we have the following lemma.

Lemma 4.7. There exists a smooth map $\varphi: U \times[0,1] \rightarrow Y^{j} \subset X_{a-1}^{a}$ such that $(\pi \circ \varphi)(s, t)=s$ and such that $\left.\varphi\right|_{U_{0}^{j} \times[0,1]}$ is a trivialization of the interval bundle $Y_{\circ}^{j}$.

Proof. If $U_{\circ}^{j}=U$, the lemma is clear, because then $Y^{j}$ is an interval bundle over $U$, which must be necessarily trivial: it admits two nowhere agreeing sections, given by the zero sets of $\rho_{a-1}$, respectively $\rho_{a}$. In general, over $U$, our bordism is isomorphic to a bordism of the form $\left((\mathbb{R} \times U) / U ; \rho_{0}, \rho_{1} ; \gamma\right)$, where $\rho_{i}(t, s)=t-\tau_{i}(s), i=0,1$, for smooth functions $\tau_{0}, \tau_{1}: U \rightarrow \mathbb{R}$ with $\tau_{0} \leq \tau_{1}$.

In that case, we have $U_{\circ}^{j}=\left\{s \in U \mid \tau_{0}(s) \neq \tau_{1}(s)\right\}$, and

$$
\varphi_{s}(t)=\tau_{0}(s)+\left(\tau_{1}(s)-\tau_{0}(s)\right) t
$$

gives the desired parametrization.

In particular, this means that the paths $\varphi_{s}:[0,1] \rightarrow X$ given by $\varphi_{s}(t):=\varphi(s, t)$ map to the fibers $\left.Y^{j}\right|_{s}$, and, for $s \in U \backslash U_{\circ}^{j}, \varphi_{s}$ is constant (since for such $s, Y_{\circ}^{j}$ is a collection of finitely many points).

Let $P(\varphi)$ be the vector bundle isomorphism between the bundles $(\gamma \circ \varphi \circ(\mathrm{id} \times i))^{*} V, i=0,1$, over $U$ given over $s \in U$ by parallel translation along the path $\varphi_{s}$. Now notice that $\varphi \circ(\mathrm{id} \times i)$, $i=0,1$ is a section of $\pi: X \rightarrow S$, with image contained in either $X_{a-1}^{a-1}$ or $X_{a}^{a}$; hence

$$
(\gamma \circ \varphi \circ(\operatorname{id} \times i))^{*} V=\left(\gamma \circ\left(\left.\pi\right|_{C} ^{-1}\right)\right)^{*} V=\left(\left.\pi\right|_{C} ^{-1}\right)^{*} \tilde{W}_{b}
$$

for some connected component $C$ of $Y^{j} \cap X_{b}^{b}$; here either $b=a-1$ or $a$. For all $s \in U$, since $\left.X_{a-1}^{a}\right|_{s}$ is a (possibly degenerate) interval, $\left.\left(V^{j} \cap X_{a-1}^{a-1}\right)\right|_{s}$ has either zero, one or two elements. Correspondingly, $\left.\left(V^{j} \cap X_{a}^{a}\right)\right|_{s}$ has two, one or zero elements. In either case, we can use the bilinear form $\beta$ to turn $P(\varphi)$ into a morphism of the required form (4.4). This defines $f^{j}$ 
in this case. As before, we use the parametrization independence as well as the fact that parallel transport preserves $\beta$ in order to show that this definition is independent of the choice of $\varphi$.

This defines $Z_{V, \nabla, \beta}$ on objects. On morphisms in 1-Bord $(M)$, we declare that $Z_{V, \nabla, \beta}$ acts by pullbacks in the obvious way. This concludes the definition of $Z_{V, \nabla, \beta}$. Of course, there are several things to check in order to show that this is a field theory. However, all checks can be made pointwise, hence are very similar to the classical arguments outlined above (cf. Construction 4.1).

Proof of Proposition 4.4. Of course, we set $\Phi(V, \nabla, \beta)=Z_{V, \nabla, \beta}$ for $(V, \nabla, \beta) \in \operatorname{Vect}_{\nabla, \beta}^{\sim}(M)$, where $Z_{V, \nabla, \beta}$ is the field theory constructed above. We now discuss how $\Phi$ acts on morphisms in $\operatorname{Vect}_{\nabla, \beta}(M)$. To this end, let $(V, \nabla, \beta)$ and $\left(V^{\prime}, \nabla^{\prime}, \beta^{\prime}\right)$ be vector bundles on $M$ with connection and a compatible bilinear form, and let $\alpha: V \rightarrow V^{\prime}$ be a vector bundle isomorphism preserving these additional structures. We now define the smooth natural transformation

$$
\eta^{\alpha}:=\Phi(\alpha): Z_{V, \nabla, \beta} \longrightarrow Z_{V^{\prime}, \nabla^{\prime}, \beta^{\prime}}
$$

First, we look at the simplicial level zero. If $X / S=\left(X / S ; \rho_{0} ; \gamma\right)$ is a single point, meaning that $X_{0}^{0}$ has connected fibers (in other words, $\pi: X_{0}^{0} \rightarrow S$ is a diffeomorphism), we have

$$
Z_{V, \nabla, \beta}(X / S)=\left(\gamma \circ\left(\left.\pi\right|_{X_{0}^{0}} ^{-1}\right)\right)^{*} V, \quad Z_{V^{\prime}, \nabla^{\prime}, \beta^{\prime}}(X / S)=\left(\gamma \circ\left(\left.\pi\right|_{X_{0}^{0}} ^{-1}\right)\right)^{*} V^{\prime} .
$$

Hence we can set

$$
\eta_{X / S}^{\alpha}:=\left(\gamma \circ\left(\left.\pi\right|_{X_{0}^{0}} ^{-1}\right)\right)^{*} \alpha
$$

in this case. Any object in 1-Bord $(M)_{0}$ can, at least locally, be uniquely decomposed into a union of single-point-objects just discussed, and hence the requirement that $\eta^{\alpha}$ is symmetric monoidal determines it on all of 1 -Bord $(M)_{0}$.

By Lemma 3.21, any smooth natural transformation $\eta: Z_{V, \nabla, \beta} \rightarrow Z_{V^{\prime}, \nabla^{\prime}, \beta^{\prime}}$ is determined by its component $\eta_{0}$ at the simplicial level zero; however, it is not clear that $\eta^{\alpha}$ defined above on simplicial level zero indeed extends to all higher simplicial levels. Here, again by Lemma 3.21 it suffices to consider the simplicial level one. To this end, let $X / S=\left(X / S ; \rho_{0}, \rho_{1} ; \gamma\right)$ be an object of $1-\operatorname{Bord}(M)_{1}$ and write $Z_{V, \nabla, \beta}(X / S)=\left(W_{0}, W_{1} ; f_{0}\right)$ and $Z_{V, \nabla, \beta}(X / S)=\left(W_{0}, W_{1} ; f_{0}\right)$. We have to check that the diagram

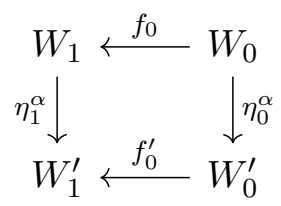

commutes, where $\eta_{i}^{\alpha}=\eta_{d_{i}^{*} X / S}^{\alpha}$, with $d_{i}:[0] \rightarrow[1]$ the usual boundary maps. Since the morphisms $f_{0}$ and $f_{0}^{\prime}$ are essentially given by parallel transport, respectively the bilinear form $\beta$, it is now easy to check that this diagram commutes for all bordisms if and only if $\alpha$ intertwines the connections and bilinear forms on $V$, respectively $V^{\prime}$.

Finally, we show that $\Phi$ is fully faithful. To this end, let $\mathrm{pt}_{M} \in 1$-Bord $(M)_{0}$ be the universal point, which is the object over $M$ given by

$$
\mathrm{pt}_{M}=\left((\mathbb{R} \times M) / M ; \rho_{0}=\operatorname{pr}_{\mathbb{R}} ; \gamma=\operatorname{pr}_{M}\right) .
$$

For any natural transformation $\eta: Z_{V, \nabla, \beta} \rightarrow Z_{V^{\prime}, \nabla^{\prime}, \beta^{\prime}}$, the component $\eta_{\mathrm{pt}_{M}}$ is a vector bundle isomorphism $V \rightarrow V$. In particular, if $\alpha: V \rightarrow V$ is a vector bundle isomorphism preserving connections and bilinear forms, tracing through the above definitions shows that $\eta_{\mathrm{pt}_{M}}^{\alpha}=\alpha$. Hence if $\eta^{\alpha}=\eta^{\alpha^{\prime}}$, this implies $\alpha=\alpha^{\prime}$; in other words, $\Phi$ is faithful. Conversely, it is easy to check that $\Phi\left(\eta_{\mathrm{pt}_{M}}\right)=\eta$ for any natural transformation $\eta$, so that $\Phi$ is also full. 


\subsection{Functors from the path category}

Let $M$ be a fixed target manifold. In this section, we restrict our attention to the smooth path category of $M$, a certain subcategory of 1-Bord $(M)$ which is somewhat easier to describe.

Definition 4.8 (smooth path category). Write $\operatorname{Path}(M)$ for the full smooth subcategory of 1 -Bord $(X)$ consisting of those objects $\left(X / S ; \rho_{0}, \ldots, \rho_{n} ; \gamma\right) \in 1$-Bord $(M)_{n}$ such that each of the sets $X_{a}^{b}, 0 \leq a \leq b \leq n$, defined in (3.6) has connected fibers. Let moreover $\operatorname{Path}_{c}(M)$ be the full subcategory of $\operatorname{Path}(M)$ consisting of those objects $\left(X / S ; \rho_{0}, \ldots, \rho_{n} ; \gamma\right)$ such that $\gamma$ is fiberwise constant in a neighborhood of $X_{a}^{a}$ for each $0 \leq a \leq n$.

Objects in $\operatorname{Path}(M)_{n}$ over $S \in$ Man can be thought of as $S$-families of paths in $M$ with $n+1$ marked points, while the full subcategory $\operatorname{Path}_{c}(M)_{n}$ consists of those paths that have sitting instants at the marked points.

Notation 4.9 (standard objects). We denote by

$$
\left(\gamma ; \tau_{0}, \ldots, \tau_{n}\right):=\left((\mathbb{R} \times S) / S ; \rho_{0}, \ldots, \rho_{n} ; \gamma\right) \in \operatorname{Path}(M)_{n}
$$

the object where $\mathbb{R} \times S \rightarrow S$ is the projection onto the second factor, $\gamma: \mathbb{R} \times S \rightarrow M$ is a smooth map, and the cut functions $\rho_{0}, \ldots, \rho_{n}$ are given by $\rho_{j}(x, s)=x-\tau_{j}(s)$ for smooth functions $\tau_{j}: S \rightarrow \mathbb{R}$ satisfying $\tau_{0} \leq \cdots \leq \tau_{n}$.

Remark 4.10. Going through the definition of the morphisms in 1-Bord $(M)$ shows that morphisms between standard objects over $S=$ pt are (equivalence classes of) diffeomorphisms $F$ of $\mathbb{R}$, which must be orientation preserving, as they need to preserve the sign of the cut functions $\rho_{i}$.

Remark 4.11. Denote by $\operatorname{Path}(M)_{n}^{\circ}$ the full subcategory of the fibered category $\operatorname{Path}(M)_{n}$ consisting of all objects $\left(\gamma ; \tau_{0}, \ldots, \tau_{n}\right)$ and the morphisms between them. This forms a prestack over Man; since any object in $\operatorname{Path}(M)_{n}$ is locally isomorphic to $\left(\gamma ; \tau_{0}, \ldots, \tau_{n}\right)$ for suitable $\gamma$ and $\tau_{a}$, the stack $\operatorname{Path}(M)_{n}$ is a stackification of $\operatorname{Path}(M)_{n}^{\circ}$. In particular, this means that any map from $\operatorname{Path}(M)_{n}$ to a smooth stack $\mathcal{V}$ is determined on $\operatorname{Path}(M)_{n}^{\circ}$ (up to unique isomorphism), and, conversely, any map from $\operatorname{Path}(M)_{n}^{\circ}$ to $\mathcal{V}$ extends, uniquely up to unique isomorphism, to $\operatorname{Path}(M)_{n}$.

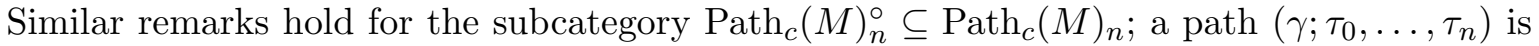
contained in $\operatorname{Path}_{c}(M)_{n}$ if for any $s \in S$ and each $j=0, \ldots, n, t \mapsto \gamma(t, s)$ is constant near $t=\tau_{j}(s)$.

Construction 4.12. Given a vector bundle $V$ with connection $\nabla$, we can define a functor

$$
Z_{V, \nabla}: \operatorname{Path}(M) \longrightarrow \text { Vect }
$$

as follows. For an $S$-family $\left(\gamma ; \tau_{0}, \ldots, \tau_{n}\right)$ of paths in $M$, we set

$$
Z\left(\gamma ; \tau_{0}, \ldots, \tau_{n}\right)=\left(W_{0}, \ldots, W_{n}, P_{1}, \ldots, P_{n}\right),
$$

where $W_{a}:=\left(\gamma \circ\left(\tau_{a} \times \mathrm{id}\right)\right)^{*} V$ is a vector bundle over $S$ and, for each $s \in S, P_{j}(s)$ is the parallel transport via $\nabla$ along the path $t \mapsto \gamma(t, s), t \in\left[\tau_{j-1}, \tau_{j}\right]$. To a morphism between two standard objects $\left(\gamma ; \tau_{0}, \ldots, \tau_{n}\right)$ and $\left(\gamma^{\prime} ; \tau_{0}^{\prime}, \ldots, \tau_{n}^{\prime}\right)$ we assign the identity; this is well-defined because automorphisms in $\operatorname{Path}(M)$ are reparametrizations of paths that fix the marked points, and parallel transport is invariant under reparametrizations. This defines the functor on the subcategory $\operatorname{Path}^{\circ}(M) \subseteq \operatorname{Path}(M)$ of standard objects; by Remark 4.11, we get a functor on all of $\operatorname{Path}(M)$, unique up to unique isomorphism. 
Now let $(V, \nabla)$ and $\left(V^{\prime}, \nabla^{\prime}\right)$ be two vector bundles with connection. Clearly, any vector bundle isomorphism $\alpha$ defines a natural transformation

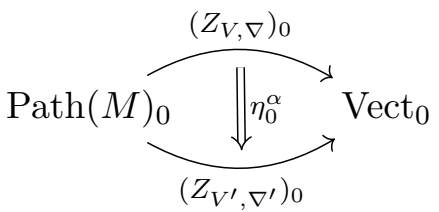

at simplicial level zero. As in the proof of Proposition 4.4, it follows that the condition for $\eta_{0}^{\alpha}$ to extend to higher simplicial levels is precisely the condition that $\alpha$ preserves connections.

Hence if $\alpha: V \rightarrow V^{\prime}$ is a connection-preserving isomorphism of vector bundles, we get a natural transformation $\eta^{\alpha}: Z_{V, \nabla} \rightarrow Z_{V^{\prime}, \nabla^{\prime}}$. This yields a functor

$$
\begin{aligned}
\Phi: \operatorname{Vect}_{\nabla}(M) & \longrightarrow \operatorname{Fun}(\operatorname{Path}(M), \text { Vect }), \\
(V, \nabla) & \longmapsto Z_{V, \nabla}, \\
\alpha & \longmapsto \eta^{\alpha} .
\end{aligned}
$$

The fundamental result is now the following.

Theorem 4.13. The functor $\Phi$ just constructed is an equivalence of categories.

Remark 4.14. At first sight, there may seem to be a clash of notations with the functor $\Phi: \operatorname{Vect}_{\nabla, \beta}(M) \rightarrow 1-\mathrm{TFT}(M)$ constructed in Section 4.1. However, it is easy to check that in fact the $\Phi$ just constructed is the composition of the functor $\Phi$ from before with the restriction functor $1-\mathrm{TFT}(M) \rightarrow \operatorname{Fun}\left(\operatorname{Path}(M)\right.$, Vect). In particular, $Z_{V, \nabla}$ is the restriction of $Z_{V, \nabla, \beta}$ to $\operatorname{Path}(M)$. Notice that the information about $\beta$ is lost in this restriction process.

Remark 4.15 (simplification). Let $\gamma, \eta: \mathbb{R} \rightarrow M$ be two smooth paths and suppose that $\gamma(t)=\eta(t)$ for $t$ in some neighborhood of $a \in \mathbb{R}$. Then the identity map of $\mathbb{R}$ induces an isomorphism $[\mathrm{id}]_{a}:(\gamma, a) \rightarrow(\eta, a)$. If now $\xi: \mathbb{R} \rightarrow M$ is a third path with $\xi(t)=\gamma(t)=\eta(t)$ for $t$ near $a$, we have the commutative diagram

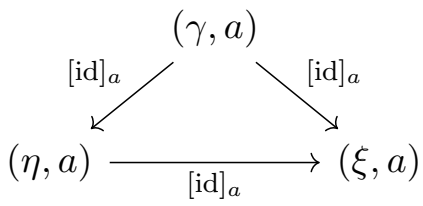

in $\operatorname{Path}(M)_{0}$. A smooth functor $Z: \operatorname{Path}(M) \rightarrow$ Vect now comes with canonical coherent isomorphisms between the vector spaces $Z(\gamma, a), Z(\eta, a)$ and $Z(\xi, a)$, given by the various $Z\left([\mathrm{id}]_{a}\right)$; this means that we can (and will) assume in the future that $Z(\gamma, a)$ is equal to $Z(\eta, a)$ for paths that are equal near $a$.

In this section, we will prove the following weaker version of Theorem 4.13, which states that $\Phi$ is an equivalence when considered as a functor to $\operatorname{Fun}\left(\operatorname{Path}_{c}(M)\right.$, Vect). The proof of Theorem 4.13 will then be completed by Proposition 4.21 from Section 4.3, which reduces the general case to the one just below.

Proposition 4.16. The composition res $\circ \Phi$ is an equivalence of categories, where

$$
\text { res: } \operatorname{Fun}(\operatorname{Path}(M), \text { Vect }) \longrightarrow \operatorname{Fun}\left(\operatorname{Path}_{c}(M), \text { Vect }\right)
$$

is the obvious restriction functor. 
We start our preparations for the proof of the above proposition with a couple of lemmas, for which we fix a smooth functor $Z: \operatorname{Path}(M) \rightarrow$ Vect. The following lemma uses Notation 4.9.

Lemma 4.17 (invertibility). For all paths $(\gamma ; a, b)$ in $\operatorname{Path}(M)_{1}$, the vector bundle map $Z(\gamma ; a, b)$ is invertible.

Proof. Invertibility can be checked pointwise, hence we may assume that $\gamma$ is a single path (i.e., a family over the point). We have $Z(\gamma ; a, a)=\mathrm{id}$ as $Z(\gamma ; a, a)$ is the image of $Z(\gamma ; a)$ under the degeneracy $[1] \rightarrow[0]$. Since the set of invertible linear maps is open and the value of $Z(\gamma ; a, b)$ depends smoothly (in particular continuously) on $a$ and $b$, we have

$$
b_{0}:=\inf \{b \mid Z(\gamma ; a, b) \text { is not invertible }\}>0 .
$$

Suppose that $b_{0}<\infty$. Then, since the set of $b$ such that $Z(\gamma ; a, b)$ is not invertible is a closed set, the infimum is actually a minimum. Therefore $Z\left(\gamma ; a, b_{0}\right)$ is not invertible, but $Z(\gamma ; a, b)$ is invertible for each $b<b_{0}$. Now

$$
Z\left(\gamma ; a, b_{0}\right)=Z\left(\gamma ; b, b_{0}\right) Z(\gamma ; a, b)
$$

for all $b \in\left[0, b_{0}\right]$. If now $b<b_{0}$, then $Z(\gamma ; a, b)$ is invertible by definition of $b_{0}$. On the other hand, since $Z\left(\gamma ; b_{0}, b_{0}\right)=\mathrm{id}$, the linear map $Z\left(\gamma ; b, b_{0}\right)$ is invertible for $b$ close enough to $b_{0}$. This leads to a contradiction to the assumption that $Z\left(\gamma ; a, b_{0}\right)$ is not invertible, as for such $b$ close to $b_{0}$, the right hand side is a composition of two invertible maps. Hence we must have $b_{0}=\infty$, which proves the lemma.

Lemma 4.18 (trivial action). For any path $\gamma$ in $M$ that is constant near a and any orientationpreserving diffeomorphism $F$ of $\mathbb{R}$ such that $F(a)=a$, the isomorphism

$$
Z\left([F]_{a}\right): Z(\gamma ; a) \longrightarrow Z(\gamma \circ F ; a)=Z(\gamma ; a)
$$

is the identity. Here, the last identity is the one given by Remark 4.15.

Proof. Let $U \ni a$ be a small neighborhood in which $\gamma$ is constant and let $G: \mathbb{R} \rightarrow \mathbb{R}$ be a diffeomorphism satisfying

$$
G(t)= \begin{cases}F(t), & t \text { near } a, \\ t, & t \notin U .\end{cases}
$$

Since $G$ has the same germ at $a$ as $F$, we have $[F]_{a}=[G]_{a}$. Moreover, $G(U)=U$, so $\gamma=\gamma \circ G$ and therefore $G$ defines an automorphism $[G]_{[a, a+1]}$ of $(\gamma, a, a+1)$. Thus, the diagram

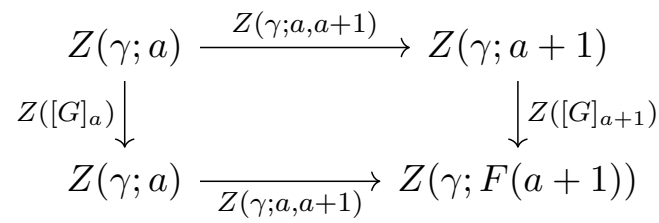

commutes. However, $Z(\gamma, a, a+1)$ is invertible by Lemma 4.17 , and $G$ is the identity near $t=a+1$, so that $Z\left([G]_{a+1}\right)=$ id. Hence $Z\left([F]_{a}\right)=Z\left([G]_{a}\right)=\mathrm{id}$.

Lemma 4.18 lets us simplify our analysis as follows. Denote by $T_{a}$ the translation diffeomorphism given by

$$
T_{a}(t)=t+a
$$


For any path $(\gamma, a)$ which is constant near $t=a$, this induces an isomorphism $\left[T_{-a}\right]_{a}:(\gamma, a) \rightarrow$ $\left(\gamma \circ T_{a}, 0\right)$. Now, let $Z: \operatorname{Path}_{c}(M) \rightarrow$ Vect be a functor that satisfies the simplifying assumption of Remark 4.15. We obtain a canonical isomorphism

$$
\mathcal{T}_{\gamma, a}:=Z\left(\left[T_{-a}\right]_{a}\right): Z(\gamma, a) \longrightarrow Z\left(\gamma \circ T_{a}, 0\right)=Z(\gamma(0), 0),
$$

as $\gamma \circ T_{a}$ has a sitting instant at $t=0$; here by abuse of notation, $\gamma(0)$ denotes the constant path equal to $\gamma(0)$. From an orientation preserving diffeomorphism $F$, we get a commutative diagram

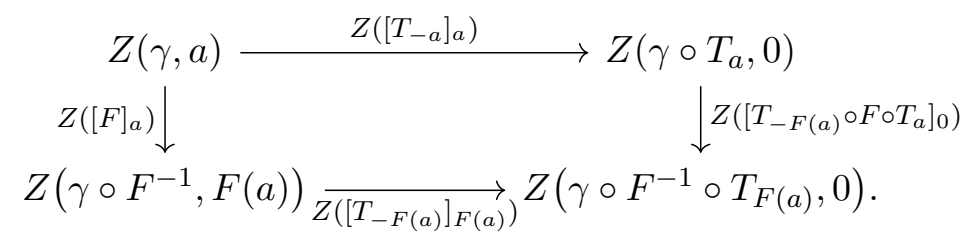

Now both $\gamma \circ T_{a}$ and $\gamma \circ F^{-1} \circ T_{F(a)}$ have a sitting instant at $t=0$, so the assumption from Remark 4.15 on $Z$ tells us that the two vector spaces in the right column agree; $Z\left(\gamma \circ T_{a}, 0\right)=$ $Z\left(\gamma \circ F^{-1} \circ T_{F(a)}, 0\right)$. A priori, $Z\left(\left[T_{-F(a)} \circ F \circ T_{a}\right]_{0}\right)$ could be a nontrivial automorphism of this vector space; however, since $\left(T_{-F(a)} \circ F \circ T_{a}\right)(0)=0$, we have $Z\left(\left[T_{-F(a)} \circ F \circ T_{a}\right]_{0}\right)=\mathrm{id}$ by Lemma 4.18. We see that the isomorphisms $\mathcal{T}_{\gamma, a}=Z\left(\left[T_{-a}\right]_{a}\right)$ satisfy the equivariance property

$$
\mathcal{T}_{\gamma, a}=\mathcal{T}_{\gamma \circ F^{-1}, F(a)} \circ Z\left([F]_{a}\right)
$$

for any diffeomorphism $F$ and all $a \in \mathbb{R}$. Using this equivariance property, we obtain the following lemma.

Lemma 4.19 (normalization). Given a strict functor $Z: \operatorname{Path}_{c}(M)^{\circ} \rightarrow$ Vect, there exists a strict functor $\tilde{Z}: \operatorname{Path}_{c}(M)^{\circ} \rightarrow$ Vect together with a natural isomorphism $\mathcal{T}: Z \rightarrow \tilde{Z}$ such that $\tilde{Z}(\gamma, a)=\tilde{Z}(\gamma(a), 0)$ for all standard objects $(\gamma, a)$. For such a functor $\tilde{Z}$

$$
Z\left([F]_{a}\right): \tilde{Z}(\gamma, a)=\tilde{Z}(\gamma(a), 0) \longrightarrow \tilde{Z}\left(\gamma \circ F^{-1}, F(a)\right)=\tilde{Z}(\gamma(a), 0)
$$

acts as the identity for any diffeomorphism $F$ of $\mathbb{R}$.

Proof. We may assume that $Z$ satisfies the simplifying assumptions of Remark 4.15. Set

$$
\tilde{Z}\left(\gamma ; \tau_{0}, \ldots, \tau_{n}\right):=\left(W_{0}, \ldots, W_{n} ; f_{1}, \ldots, f_{n}\right),
$$

with $W_{j}:=Z\left(\gamma\left(\tau_{j}\right), 0\right)$, and

$$
f_{j}:=\mathcal{T}_{\gamma, \tau_{j}} \circ Z\left(\gamma ; \tau_{j-1}, \tau_{j}\right) \circ \mathcal{T}_{\gamma, \tau_{j-1}}^{-1},
$$

where the $\mathcal{T}_{\gamma, a}$ are defined as in (4.7). Moreover, for a diffeomorphism $F$, set $Z\left([F]_{\left[\tau_{0}, \tau_{n}\right]}\right):=$ id. By the equivariance property (4.8), this gives a well-defined functor $\tilde{Z}: \operatorname{Path}_{c}(M)^{\circ} \rightarrow \operatorname{Vect}^{\circ}$

Finally, let $\mathcal{T}$ be given by

$$
\mathcal{T}_{\left(\gamma ; \tau_{0}, \ldots, \tau_{n}\right)}=\left(\mathcal{T}_{\gamma, \tau_{0}}, \ldots, \mathcal{T}_{\gamma, \tau_{n}}\right)
$$

This clearly defines a natural transformation $Z \rightarrow \tilde{Z}$. That $\tilde{Z}\left([F]_{a}\right)$ acts as the identity also follows directly from (4.8).

We are now in a position to prove the main result of this section. 
Proof of Proposition 4.16. That res $\circ \Phi$ is full and faithful is shown just as in the proof of Proposition 4.4. It therefore remains to show that res $\circ \Phi$ is essentially surjective. Moreover, it suffices to consider the functor on the subcategory of standard objects.

Let $Z: \operatorname{Path}_{c}(M)^{\circ} \rightarrow$ Vect be a strict smooth functor. We assume moreover that $Z$ is normalized in the sense of Lemma 4.19; in other words, $Z(\gamma, a)=Z(\gamma(a), 0)$ for all paths $\gamma$ and all $a \in \mathbb{R}$, and $Z\left([F]_{a}\right)=$ id for all diffeomorphisms $F$ on $\mathbb{R}$. In particular, this implies that

$$
Z(\gamma \circ F ; a, b)=Z(\gamma ; F(a), F(b))
$$

as map from $Z(\gamma(a), 0)$ to $Z(\gamma(b), 0)$.

First let us extract a vector bundle from $Z$. To this end, let $\mathrm{pt}_{M}$ be the universal point introduced in (4.5). Note that $\mathrm{pt}_{M} \in \operatorname{Path}_{c}(M)$ and set $V:=Z\left(\mathrm{pt}_{M}\right)$. For any path $(\gamma, a)$, our assumption on $Z$ then implies that $Z(\gamma, a)=V_{\gamma(a)}$. Now in general for objects $\left(\gamma ; \tau_{0}, \ldots, \tau_{n}\right) \in$ $\operatorname{Path}_{c}(M)_{n}^{\circ}$, we have

$$
Z\left(\gamma ; \tau_{0}, \ldots, \tau_{n}\right)=\left(W_{0}, \ldots, W_{n} ; f_{1}, \ldots, f_{n}\right) .
$$

If $d_{i}:[0] \rightarrow[n], i=0, \ldots, n$, is the map with image $i \in[n]$, we have

$$
W_{i}=d_{i}^{*} Z\left(\gamma ; \tau_{0}, \ldots, \tau_{n}\right)=Z\left(d_{i}^{*}\left(\gamma ; \tau_{0}, \ldots, \tau_{n}\right)\right)=Z\left(\gamma ; \tau_{i}\right)=V_{\gamma\left(\tau_{i}\right)},
$$

hence the $W_{i}$ are already determined by $V$.

It remains to determine the vector bundle maps $f_{i}$; we will use Proposition 4.3 for this. To obtain a section $P$ as in the proposition, we use modification functions, which are defined as follows.

Definition 4.20 (modication function). A two-sided modification function is a smooth function $\chi: \mathbb{R} \rightarrow[0,1]$ such that

(1) $\chi$ is nondecreasing,

(2) $\chi(t)=0$ for $t$ near zero,

(3) $\chi(t)=1$ for $t$ near one.

By $\chi_{a, b}, a \leq b$ we denote the function given by

$$
\chi_{a, b}(t):=a+(b-a) \chi\left(\frac{t-a}{b-a}\right)
$$

for $t \in \mathbb{R}$. This function is then only nonconstant on $[a, b]$ and takes values in $[a, b]$.

Later, in Definition 5.5, we will introduce also left and right modification functions, as well as their family versions. For now, we drop the adjective "two-sided". If $\chi$ is a modification function and $\gamma \in C^{\infty}([0,1], M)$, then $\gamma \circ \chi$ is a path that is defined on all of $\mathbb{R}$ and which is constant on $(-\infty, \varepsilon] \cup[1-\varepsilon, \infty)$ for some $\varepsilon>0$. Hence

$$
P(\gamma):=Z(\gamma \circ \chi ; 0,1)
$$

is well-defined for each $\gamma \in C^{\infty}([0,1], X)$. Note that $P(\gamma)$ maps $Z(\gamma \circ \chi, 0)=Z(\gamma(0) ; 0)=V_{\gamma(0)}$ to $Z(\gamma \circ \chi ; 1)=Z(\gamma(1) ; 1)=V_{\gamma(1)}$. This construction works in families and therefore we get a smooth section $P$ of the bundle $\mathrm{ev}_{0}^{*} V^{\vee} \otimes \mathrm{ev}_{1}^{*} V$, as required. The crucial result, which will be shown in Lemma 4.23 below, is then that $P(\gamma)$ is independent of the choice of modification function. 
We need to check that $P$ is multiplicative, in the sense of (4.2). For this, we must use the next simplicial level. For $a \in[0,1]$ fixed, define $\xi: \mathbb{R} \rightarrow[0,1]$ by

$$
\xi(t):= \begin{cases}a \chi(t), & t \in[0,1], \\ a+(1-a) \chi(t-1), & t \in[1,2] .\end{cases}
$$

Then $\xi^{\prime}(t) \geq 0$ everywhere, and $\xi$ is constant near $t=0,1,2$ (with values $0, a, 1$ ). Hence $t \mapsto \xi_{2}(t):=\xi(2 t)$ is a modification function in the sense of Definition 4.20. Therefore, by independence of the modification function (Lemma 4.23 below), we have

$$
P(\gamma)=Z(\gamma \circ \chi ; 0,1)=Z\left(\gamma \circ \xi_{2} ; 0,1\right)=Z(\gamma \circ \xi ; 0,2)
$$

Notice that in the last step, we used the parametrization independence (4.9).

Now, since $\gamma \circ \xi$ is constant near $1,(\gamma \circ \xi ; 0,1)$ and $(\gamma \circ \xi ; 1,2)$ are also objects of $\operatorname{Path}_{c}(M)$, and we get

$$
Z(\gamma \circ \xi ; 0,2)=Z(\gamma \circ \xi ; 1,2) \circ Z(\gamma \circ \xi ; 0,1)
$$

However, by definition, we have

$$
(\gamma \circ \xi)(t)= \begin{cases}\left(\mathfrak{s}_{0, a} \gamma \circ \chi\right)(t), & t \in[0,1], \\ \left(\mathfrak{s}_{a, 1} \gamma \circ \chi\right)(t-1), & t \in[1,2] .\end{cases}
$$

Hence, $Z(\gamma \circ \xi ; 0,1)=P\left(\mathfrak{s}_{0, a} \gamma\right)$ and, again by $(4.9)$,

$$
Z(\gamma \circ \xi ; 1,2)=Z\left(\mathfrak{s}_{a, 1} \gamma \circ \chi \circ T_{-1} ; 1,2\right)=Z\left(\mathfrak{s}_{a, 1} \gamma \circ \chi ; 0,1\right)=P\left(\mathfrak{s}_{a, 1} \gamma\right)
$$

Therefore,

$$
P(\gamma)=P\left(\mathfrak{s}_{a, 1} \gamma\right) P\left(\mathfrak{s}_{0, a} \gamma\right),
$$

that is, $P$ is indeed multiplicative. By Proposition 4.3, there exists a connection $\nabla$ on $V$ such that $P(\gamma)$ is given by parallel transport along $\gamma$ with respect to $\nabla$.

To conclude the argument, let $s_{i}:[1] \rightarrow[n], i=1, \ldots, n$ be the map with $s_{i}(0)=i-1$ and $s_{i}(1)=i$, and notice that

$$
f_{i}=s_{i}^{*} Z\left(\gamma ; \tau_{0}, \ldots, \tau_{n}\right)=Z\left(s_{i}^{*}\left(\gamma ; \tau_{0}, \ldots, \tau_{n}\right)\right)=Z\left(\gamma ; \tau_{i-1}, \tau_{i}\right) .
$$

Define $\tilde{\gamma}$ by $\tilde{\gamma}(t):=\gamma\left(\tau_{i-1}+\left(\tau_{i}-\tau_{i-1}\right) t\right)$; then $Z\left(\gamma ; \tau_{i-1}, \tau_{i}\right)=Z(\tilde{\gamma} ; 0,1)$, once more by $(4.9)$. Since $\tilde{\gamma}$ has sitting instants at $t=0$ and 1 , we have $\tilde{\gamma}=\tilde{\gamma} \circ \chi$ for a suitable modification function (just choose $\chi$ in such a way that $\chi(t)=t$ wherever $\tilde{\gamma}$ is not constant). Finally, we get

$$
f_{j}=Z(\tilde{\gamma} ; 0,1)=Z(\tilde{\gamma} \circ \chi ; 0,1)=P(\tilde{\gamma}) .
$$

Since parallel transport is parametrization independent, this coincides with the parallel transport along $\gamma$ from $\tau_{i-1}$ to $\tau_{i}$, with respect to the connection $\nabla$. Hence $Z$ coincides with the restriction of $Z_{V, \nabla}=\Phi(V, \nabla)$ to $\operatorname{Path}_{c}(M)$, where $Z_{V, \nabla}$ is the functor from Construction 4.12.

\subsection{General paths}

In this section, we finish the proof of Theorem 4.13. Having Proposition 4.16 at hand, this will be achieved by establishing the following result. 
Proposition 4.21. The functor res from the previous section is an equivalence of categories. More specifically, there is a functor

$$
\text { ext: } \operatorname{Fun}\left(\operatorname{Path}_{c}(M), \text { Vect }\right) \longrightarrow \operatorname{Fun}(\operatorname{Path}(M), \text { Vect })
$$

such that res $\circ$ ext $=\mathrm{id}$, together with a natural isomorphism $\eta$ : id $\rightarrow$ ext $\circ$ res.

We need several lemmas, for which we fix a smooth functor $Z: \operatorname{Path}(M) \rightarrow$ Vect. We will assume that $Z$ is a strict functor (which is possible by Lemma 3.37), and we will also make the simplifying assumptions discussed in Remark 4.15.

Lemma 4.22 (introducing a sitting instant). Fix numbers $a \leq b$ and let $F: \mathbb{R} \rightarrow \mathbb{R}$ be a smooth monotonically increasing function such that $F(t)=t$ for $t$ near $a$ and near $b$. Then, for all paths $\gamma: \mathbb{R} \rightarrow M$, we have $Z(\gamma ; a, b)=Z(\gamma \circ F ; a, b)$.

We remark that if we choose $F$ to be constant somewhere in between $a$ and $b$ (as allowed by the lemma), the path $\gamma \circ F$ will have a sitting instant somewhere in between $a$ and $b$.

Proof. If $F^{\prime}(x) \neq 0$ for all $x \in[a, b]$, so that $F$ is a diffeomorphism onto its image in a neighborhood of $[a, b]$, the simplicial structure yields the commutative diagram

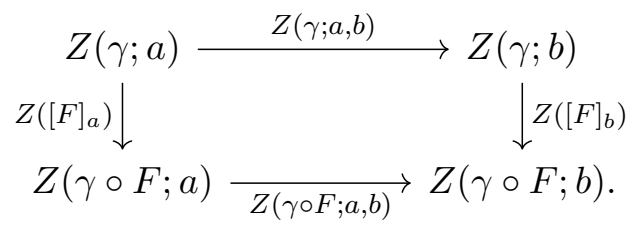

This, together with the fact that $F=$ id near $a$ and $b$, so that $Z(\gamma \circ F ; a)=Z(\gamma ; a)$ and $Z\left([F]_{a}\right)=\mathrm{id}$, and similarly for $b$, proves the result in this case.

The general case now follows from the fact that

$$
F_{\varepsilon}(t):=(1-\varepsilon) F(t)+\varepsilon t
$$

is a one-parameter family of maps such that $F_{0}=F$ and such that $F_{\varepsilon}$ is a diffeomorphism whenever $\varepsilon \in(0,1]$. Therefore, by the observations above, we have $Z(\gamma ; a, b)=Z\left(\gamma \circ F_{\varepsilon} ; a, b\right)$ for all $\varepsilon \in(0,1]$, and, by continuity, the equality persists for $\varepsilon=0$.

We are now able to prove the following essential lemma, which proves the independence of the choice of modification function (see Definition 4.20).

Lemma 4.23 (independence of $\chi$ ). Let $Z: \operatorname{Path}_{c}(X) \rightarrow$ Vect be a functor. Then for any two modification functions $\chi, \tilde{\chi}$, we have

$$
Z\left(\gamma \circ \chi_{a, b}, a, b\right)=Z\left(\gamma \circ \tilde{\chi}_{a, b}, a, b\right)
$$

as morphisms from $Z(\gamma(a), a)$ to $Z(\gamma(b), b)$.

Proof. Clearly, we may assume for simplicity that $a=0$ and $b=1$. Now, we first argue that we may furthermore assume that $\chi(t)=\tilde{\chi}(t)=t$ for $t$ in a neighborhood of $\frac{1}{2}$. Since $\chi$ is not constant, there exists some $t_{0} \in(0,1)$ such that $\chi^{\prime}\left(t_{0}\right) \neq 0$. We may arrange that $t_{0}=\frac{1}{2}$ : Choose some diffeomorphism $F$ that is the identity near $t=0,1$ and sends $\frac{1}{2}$ to $t_{0}$. Then $\xi:=\chi \circ F$ is again a modification function, now satisfying $\xi^{\prime}\left(\frac{1}{2}\right) \neq 0$. We get that there exists a neighborhood of $\frac{1}{2}$, where $\xi^{\prime}$ is invertible. Hence there exists some small $\varepsilon>0$ such that we can find a diffeomorphism $G$ of $\mathbb{R}$ with

$$
G(t)= \begin{cases}t & \text { if } \quad t \in(-\infty, \varepsilon] \cup[1-\varepsilon, \infty), \\ \xi^{-1}(t) & \text { if } \quad t \in\left[\frac{1}{2}-\varepsilon, \frac{1}{2}+\varepsilon\right] .\end{cases}
$$


Then $\xi \circ G$ is a modification function that is the identity near $t=\frac{1}{2}$, and we have

$$
Z(\gamma \circ(\xi \circ G) ; 0,1)=Z(\gamma \circ \chi \circ(F \circ G) ; 0,1)=Z(\gamma \circ \chi ; 0,1)
$$

since $F \circ G$ is the identity near $t=0,1$.

By the above, after replacing $\chi$ and $\tilde{\chi}$ with equivalent modification functions, we may assume that $\chi(t)=\tilde{\chi}(t)=t$ near $t=\frac{1}{2}$. Now let $F$ and $\tilde{F}$ be monotonic functions on $\mathbb{R}$ such that

(1) $F(t)=\tilde{F}(t)=t$ on $\left(-\infty,-\frac{1}{2}+\varepsilon\right]$,

(2) $F(t)=\tilde{F}(t)$ on $(-\infty, 0]$,

(3) $F(t)=\chi(t)$ and $\tilde{F}(t)=\tilde{\chi}(t)$ on $[0, \infty)$.

This is possible since $\chi$ and $\tilde{\chi}$ are both constant near zero. Now since $F, \tilde{F}$ are the identity near $-\frac{1}{2}$ and $\frac{1}{2}$, Lemma 4.22 gives

$$
Z\left(\gamma \circ F ;-\frac{1}{2}, \frac{1}{2}\right)=Z\left(\gamma ;-\frac{1}{2}, \frac{1}{2}\right)=Z\left(\gamma \circ \tilde{F} ;-\frac{1}{2}, \frac{1}{2}\right)
$$

On the other hand,

$$
\begin{aligned}
& Z\left(\gamma \circ F ;-\frac{1}{2}, \frac{1}{2}\right)=Z\left(\gamma \circ \chi ; 0, \frac{1}{2}\right) \circ Z\left(\gamma \circ F ;-\frac{1}{2}, 0\right), \\
& Z\left(\gamma \circ \tilde{F} ;-\frac{1}{2}, \frac{1}{2}\right)=Z\left(\gamma \circ \tilde{\chi} ; 0, \frac{1}{2}\right) \circ Z\left(\gamma \circ F ;-\frac{1}{2}, 0\right),
\end{aligned}
$$

by the construction of $F$ and $\tilde{F}$. As all morphisms involved are invertible in view of Lemma 4.17, combining (4.10) with (4.11) implies that $Z\left(\gamma \circ \chi ; 0, \frac{1}{2}\right)=Z\left(\gamma \circ \tilde{\chi} ; 0, \frac{1}{2}\right)$. A similar argument shows that $Z\left(\gamma \circ \chi ; \frac{1}{2}, 1\right)=Z\left(\gamma \circ \tilde{\chi} ; \frac{1}{2}, 1\right)$; combining these observations finishes the proof.

We are now ready to give the proof of the main result of this section.

Proof of Proposition 4.21. We first define the functor ext. For a smooth functor

$$
Z: \operatorname{Path}_{c}(M) \rightarrow \text { Vect }
$$

we set $\operatorname{ext} Z(\gamma, a):=Z(\gamma(a) ; a)$ and

$$
\operatorname{ext} Z\left(\gamma ; \tau_{0}, \ldots, \tau_{n}\right)=Z\left(\bar{\gamma} ; \tau_{0}, \ldots, \tau_{n}\right) \text {, }
$$

where $\bar{\gamma}$ is given on the interval $\left[\tau_{j-1}, \tau_{j}\right]$ by

$$
\bar{\gamma}(t)=\left(\gamma \circ \chi_{\tau_{i-1}, \tau_{i}}\right)(t)
$$

for some modification function $\chi$. By Lemma 4.23, this is independent of the choice of modification function. Let $F$ be a diffeomorphism of $\mathbb{R}$, which defines a morphism

$$
[F]_{a}:(\gamma \circ F, a) \longrightarrow(\gamma, F(a))
$$

in $\operatorname{Path}(M)_{0}$. Let $T_{a}, a \in \mathbb{R}$ be the translations defined in (4.6) above; we then set

$$
\operatorname{ext} Z\left([F]_{a}\right):=Z\left(\left[T_{F(a)-a}\right]_{a}:(\gamma(F(a)), a) \rightarrow(\gamma(F(a)), F(a))\right) .
$$

Using the simplicial structure and Remark 4.11, this determines the functor ext $Z$ completely. 
In order to show that ext $Z$ is well defined, we need to check functoriality. Let $F$ be a diffeomorphism of $\mathbb{R}$, which defines an automorphism

$$
[F]_{[a, b]}:(\gamma \circ F ; a, b) \longrightarrow\left(\gamma ; a^{\prime}, b^{\prime}\right), \quad \text { where } \quad a^{\prime}:=F(a), \quad b^{\prime}:=F(b),
$$

in $\operatorname{Path}(M)_{q}$. We need to show that the square

$$
\begin{array}{cr}
Z((\gamma \circ F)(a), a) \stackrel{Z(\overline{\gamma \circ F} ; a, b)}{\longrightarrow} Z((\gamma \circ F)(b) ; b) \\
Z\left(\left[T_{a^{\prime}-a}\right]_{a}\right) \downarrow \\
Z\left(\gamma\left(a^{\prime}\right), a^{\prime}\right) \stackrel{\downarrow}{\longrightarrow} \underset{\left(\left[T_{b^{\prime}-b}\right]_{b}\right)}{\longrightarrow\left(\bar{\gamma} ; a^{\prime}, b^{\prime}\right)} & Z\left(\gamma\left(b^{\prime}\right), b^{\prime}\right)
\end{array}
$$

commutes. We have

$$
Z(\overline{\gamma \circ F} ; a, b)=Z\left(\gamma \circ F \circ \chi_{a, b} ; a, b\right)=Z\left([F]_{b}\right)^{-1} Z\left(\gamma \circ F \circ \chi_{a, b} \circ F^{-1} ; a^{\prime}, b^{\prime}\right) Z\left([F]_{a}\right) .
$$

Now first notice that $F \circ \chi_{a, b} \circ F^{-1}=\tilde{\chi}_{a^{\prime}, b^{\prime}}$ for some modification function $\tilde{\chi}$, hence the middle term equals $Z\left(\bar{\gamma} ; a^{\prime}, b^{\prime}\right)$ (here, of course, we use Lemma 4.23 again). Secondly,

$$
Z\left([F]_{a}\right)=Z\left(\left[T_{a^{\prime}-a}\right]_{a}\right) Z\left(\left[T_{a^{\prime}-a}^{-1} \circ F\right]_{a}\right),
$$

with $Z\left(\left[T_{a^{\prime}-a}^{-1} \circ F\right]_{a}\right)=$ id by Lemma 4.18, as $\left(T_{a^{\prime}-a}^{-1} \circ F\right)(a)=a$. Using a similar argument for $Z\left([F]_{b}\right)$, we obtain

$$
Z(\overline{\gamma \circ F} ; a, b)=Z\left(\left[T_{b^{\prime}-b}\right]_{b}\right)^{-1} Z\left(\bar{\gamma} ; a^{\prime}, b^{\prime}\right) Z\left(\left[T_{a^{\prime}-a}\right]_{a}\right),
$$

which was the claim.

If $\eta: Z \rightarrow Z^{\prime}$ is a smooth natural transformation between smooth functors $Z, Z^{\prime}: \operatorname{Path}_{c}(M) \rightarrow$ Vect, we set

$$
(\operatorname{ext} \eta)_{(\gamma, a)}:=\eta_{(\gamma(a), a)} .
$$

It is straightforward to check that this gives a natural transformation ext $\eta$ : $\operatorname{ext} Z \rightarrow \operatorname{ext} Z^{\prime}$ and that the assignment $\eta \mapsto \operatorname{ext} \eta$ is functorial in $\eta$. This finishes the definition of ext.

To see that res o ext $=\mathrm{id}$, we have to check that for paths $\gamma$ that already have sitting instants at $\tau_{0}, \ldots, \tau_{n}$, one has $Z\left(\gamma ; \tau_{0}, \ldots, \tau_{n}\right)=Z\left(\bar{\gamma} ; \tau_{0}, \ldots, \tau_{n}\right)$. However, for such a path $\gamma$, there exists a modification function $\chi$ such that on each subinterval $\left[\tau_{j-1}, \tau_{j}\right]$, we have $\gamma=\gamma \circ \chi_{\tau_{j-1}, \tau_{j}}$. Moreover, the composition of two modification functions is again a modification function, hence the claim follows once more from Lemma 4.23.

Finally, we construct a natural isomorphism $\eta$ : id $\rightarrow$ ext $\circ$ res. To this end, choose numbers $0<\delta<\ell$ and a modification function $\chi$ such that $\chi(t)=t$ for $t$ near $\delta$ and set

$$
\eta_{\gamma, a}:=Z\left(\gamma \circ \chi_{a, a+\ell} ; a, a+\delta\right)^{-1} \circ Z(\gamma ; a, a+\delta): Z(\gamma ; a) \longrightarrow Z(\gamma(a), a) .
$$

We claim that this definition is independent of the modification function $\chi$ and the choice of $\delta$ and $\ell$. For notational simplicity, let $a=0$ and suppose $\ell=1$; the case $a \neq 0$ is similar. Now, let $\tilde{\chi}$ be another modification function also satisfying $\tilde{\chi}(t)=t$ for $t$ near $\delta$. Since the values of a modification function on $[\delta, 1]$ are irrelevant for the definition of $\eta$, we may as well assume that $\tilde{\chi}=\chi$ on $[\delta, 1]$ (which then implies that they in fact agree on a neighborhood of $[\delta, 1]$ ). Now, by Lemma 4.23 , we have $Z(\gamma \circ \chi ; 0,1)=Z(\gamma \circ \tilde{\chi} ; 0,1)$. On the other hand

$$
Z(\gamma \circ \chi ; 0,1)=Z(\gamma \circ \chi ; \delta, 1) \circ Z(\gamma \circ \chi ; 0, \delta)=Z(\gamma \circ \tilde{\chi} ; \delta, 1) \circ Z(\gamma \circ \chi ; 0, \delta)
$$


since $\chi$ and $\tilde{\chi}$ agree in a neighborhood of $\delta$. But this equals

$$
Z(\gamma \circ \tilde{\chi} ; 0,1)=Z(\gamma \circ \tilde{\chi} ; \delta, 1) \circ Z(\gamma \circ \tilde{\chi} ; 0, \delta),
$$

from which we obtain the desired equality $Z(\gamma \circ \chi ; 0, \delta)=Z(\gamma \circ \tilde{\chi} ; 0, \delta)$, by virtue of Lemma 4.17. To see the independence from $\delta$, let $\tilde{\chi}$ be a modification function with $\tilde{\chi}(t)=t$ near $\tilde{\delta}$. Without loss of generality, suppose that $\tilde{\delta}<\delta$. By the first step, we are free to choose the modification function $\chi$ any way we like, under the constraint that $\chi(t)=t$ near $\delta$. We now choose it in such a way that in fact $\chi(t)=t$ in a neighborhood of the interval $[\tilde{\delta}, \delta]$. Then

$$
Z(\gamma \circ \chi ; 0, \delta)=Z(\gamma \circ \chi ; \tilde{\delta}, \delta) \circ Z(\gamma \circ \chi ; 0, \tilde{\delta}) .
$$

However, on $[\delta, \tilde{\delta}]$, we have $\gamma \circ \chi=\gamma$, and by the first step, we have $Z(\gamma \circ \chi ; 0, \tilde{\delta})=Z(\gamma \circ \tilde{\chi} ; 0, \tilde{\delta})$, so that

$$
Z(\gamma \circ \chi ; 0, \delta)=Z(\gamma ; \tilde{\delta}, \delta) \circ Z(\gamma \circ \tilde{\chi} ; 0, \tilde{\delta}) .
$$

This finishes the argument that $\eta_{\gamma, a}$ does not depend on $\delta$. The independence of the choice of $\ell$ now follows immediately.

In order to show that $\eta$ indeed gives rise to a natural transformation, we have to show that for any diffeomorphism $F$ of $\mathbb{R}$, we have the equivariance property

$$
\eta_{\gamma \circ F, a}=\eta_{\gamma, F(a)} \circ Z\left([F]_{a}\right) .
$$

First notice that for any path $\xi$ and any diffeomorphism $F$, we have a commuting square

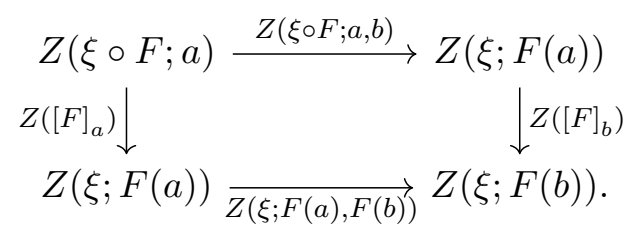

Applying this to $\xi=\gamma \circ F \circ \chi_{a, a+1} \circ F^{-1}$, we have

$$
\begin{aligned}
\eta_{\gamma \circ F, a} & =Z(\xi \circ F ; a, a+\delta)^{-1} \circ Z(\gamma \circ F ; a, a+\delta) \\
& =Z\left([F]_{a}\right)^{-1} \circ Z(\xi ; F(a), F(a+\delta))^{-1} \circ Z(\gamma ; F(a), F(a+\delta)) \circ Z\left([F]_{a}\right)
\end{aligned}
$$

Notice that the two appearances of $Z\left([F]_{a}\right)$ above in fact denote different things: The one on the right is an isomorphism $Z(\gamma \circ F ; a+\delta) \rightarrow Z(\gamma ; F(a+\delta))$, while the one on the left is an isomorphism $Z(\xi \circ F ; a) \rightarrow Z(\xi ; F(a))$. The latter is trivial by Lemma 4.18 since $\xi \circ F$ is constant near $a$ and $\xi$ is constant near $F(a)$. Finally, it is easy to see that $F \circ \chi_{a, a+1} \circ F^{-1}=$ $\tilde{\chi}_{F(a), F(a+1)}$ for some modification function $\tilde{\chi}$. Hence the last expression equals $\eta_{\gamma, F(a)} \circ Z\left([F]_{a}\right)$, which finishes the proof of identity (4.12).

Now the functions $\eta_{\gamma, a}$ give a natural transformation $\eta$ : id $\rightarrow$ ext $\circ$ res as follows. For each functor $Z: \operatorname{Path}_{c}(M) \rightarrow$ Vect, we need to give a natural transformation $\eta^{Z}: Z \rightarrow \operatorname{ext}\left(\left.Z\right|_{\operatorname{Path}_{c}(M)}\right)$. The components of this natural transformation are given by

$$
\eta_{\left(\gamma ; \tau_{0}, \ldots, \tau_{n}\right)}^{Z}=\left(\eta_{\gamma, \tau_{0}}, \ldots, \eta_{\gamma, \tau_{n}}\right),
$$

with the $\eta_{\gamma, \tau_{j}}$ as constructed above. This gives an isomorphism from

$$
Z\left(\gamma ; \tau_{0}, \ldots, \tau_{n}\right) \longrightarrow \operatorname{ext} Z\left(\gamma ; \tau_{0}, \ldots, \tau_{n}\right)=Z\left(\bar{\gamma} ; \tau_{0}, \ldots, \tau_{n}\right),
$$

as required. The equivariance property (4.12) shows that these isomorphisms indeed fit together to give a natural transformation.

The last thing to show is that if $\mu: Z \rightarrow Y$ is a smooth natural transformation of smooth functors $Y, Z: \operatorname{Path}_{c}(M) \rightarrow$ Vect, then $\eta^{Y} \circ \mu=\operatorname{ext} \mu \circ \eta^{Z}$. But this is trivial, since $\eta^{Z}$ and $\eta^{Y}$ act as the identity on $\operatorname{Path}_{c}(M)$ and ext $\left.\mu\right|_{\operatorname{Path}_{c}(M)}=\mu$. 


\subsection{Proof of the classification theorem}

We are now in a position to prove Theorem 4.2; more precisely, we will prove that the functor $\Phi$ from Proposition 4.4 is essentially surjective.

Proof of Theorem 4.2. Let $Z: 1$-Bord $(M) \rightarrow$ Vect be a field theory. By the results of the previous section, we may assume that $\left.Z\right|_{\operatorname{Path}(M)}=\Phi(V, \nabla)$, where

$$
\Phi: \operatorname{Fun}(\operatorname{Path}(M), \operatorname{Vect}) \longrightarrow \operatorname{Vect} \tilde{\nabla}(M)
$$

is the equivalence constructed in Section 4.2. This means that $Z(\gamma, a)=V_{\gamma(a)}$ for all paths $\gamma$ in $M$ and all $a$, and that $Z(\gamma ; a, b)$ is given by parallel transport along $\gamma$ from $a$ to $b$, with respect to the connection $\nabla$.

To get a bilinear form $\beta$ on $V$, consider the constant right elbow, which is the bordism $R=\left((\mathbb{R} \times M) / M ; \rho_{0}, \rho_{1} ; \gamma_{\text {const }}\right)$, where $\gamma_{\text {const }}(t, p)=p, \rho_{1} \equiv-1$ and $\rho_{0}(t, p):=t(1-t)$. Then, canonically,

$$
d_{0}^{*} R \cong\left(\gamma_{\text {const }}, 0\right) \amalg\left(\gamma_{\text {const }}, 1\right) \quad \text { and } \quad d_{1}^{*} R=\varnothing .
$$

Hence $Z(R)$ is a linear map from $Z\left(\gamma_{\text {const }}, 0\right) \otimes Z\left(\gamma_{\text {const }}, 1\right)$ to $\mathbb{K}$. However, $Z\left(\gamma_{\text {const }}, 0\right)=$ $Z\left(\gamma_{\text {const }}, 1\right)=V$, so we get a bilinear form $\beta:=Z(R)$ on $V$. It is symmetric because the diffeomorphism $F: \mathbb{R} \rightarrow \mathbb{R}$,

$$
F(t)=\frac{1}{2}-t
$$

determines an automorphism of $R$ in 1-Bord $(M)$, the restriction of which to $R_{0}^{0}$ gets mapped to the symmetry isomorphism of $V \otimes V$ under $Z$. This is because $F$ swaps the two components of $R_{0}^{0}=(\{0,1\} \times M) / M$.

To see that $\beta$ is nondegenerate, let $L$ be the constant left elbow, which is the bordism given by $L=\left(\mathbb{R} \times M / M ; \vartheta_{0}, \vartheta_{1} ; \gamma_{\text {const }}\right)$, where $\vartheta_{0}(t) \equiv 1$ and $\vartheta_{1}(t)=-\rho_{0}(t)$. Set $\tau:=Z(L)$, which is a section of the bundle $\operatorname{Hom}(\mathbb{K}, V \otimes V)$, i.e., a section of $V \otimes V$. The "snake identity"

$$
(\mathrm{id} \otimes \beta) \circ(\tau \otimes \mathrm{id})=\mathrm{id}
$$

is satisfied. (To be precise, the left hand side is in fact a map from $\mathbb{K} \otimes V$ to $V \otimes \mathbb{K}$, but this can be canonically be identified with an endomorphism of $V$; the requirement is that this endomorphism be the identity.) We now analyze this identity on each fiber; to this end, write $\tau_{p}=\sum_{i j} v_{i} \otimes w_{j}$ for some elements $v_{i}, w_{j} \in V_{p}$. Then for any $u \in V_{p}$, we have

$$
(\mathrm{id} \otimes \beta) \circ(\tau \otimes \mathrm{id})(1 \otimes u)=\sum_{i, j}(\mathrm{id} \otimes \beta)\left(v_{i} \otimes w_{j} \otimes u\right)=\sum_{i, j} \beta\left(w_{j}, u\right) v_{i} \otimes 1 .
$$

The requirement that this be equal to $u \otimes 1$ implies that $\beta$ must be nondegenerate, as claimed. Note that it also implies that $\tau=\sum_{j} \varepsilon_{j} b_{j} \otimes b_{j}$, where $b_{1}, \ldots, b_{n}$ is a generalized orthonormal basis for $\beta$; thus, $\epsilon$ is determined by $\beta$.

We now want to show that $Z=Z_{V, \nabla, \beta}=\Phi(V, \nabla, \beta)$, where $\Phi$ denotes the functor constructed in Proposition 4.4. We already know that $Z=Z_{V, \nabla, \beta}$ on simplicial level zero and $Z(B)=$ $Z_{V, \nabla, \beta}(B)$ on all bordisms $B$ that are (tensor products of) intervals and/or constant elbows. Using the techniques from the previous section, we can introduce sitting instants into any nonconstant elbow $B$ and then express $B$ as the composition of two intervals and a constant elbow. This determines the field theory on all intervals and elbows, as well as on circles, since any circle can be decomposed into two elbows. Hence we have $Z=\Phi(V, \nabla, \beta)$. 


\subsection{The oriented case}

We now briefly comment on the oriented case. The geometry considered here is the one considered in Example 3.35, where manifolds are endowed with orientations. In dimension one, the resulting bordism category will be denoted 1-Bord ${ }^{\text {or }}(M)$ and we write $1-\operatorname{TFT}^{\text {or }}(M)$ for the corresponding groupoid of field theories.

The main difference of 1 -Bord ${ }^{\text {or }}(M)$ to the unoriented bordism category is that there are now two different kinds of points: Remember that a point is given as the zero set of the cut function $\rho_{0}$ on a one-dimensional manifold (respectively a family of such). Now because $\rho_{0}(x)=0$ for $x \in X$, the orientation allows to ask whether $\mathrm{d} \rho_{0}(x)$ (which is non-zero and hence a basis of $T_{x} X$ ) is positively oriented or negatively oriented. This leads to positive respectively negative points. Correspondingly, we have one more elementary bordism, as displayed in Figure 5.

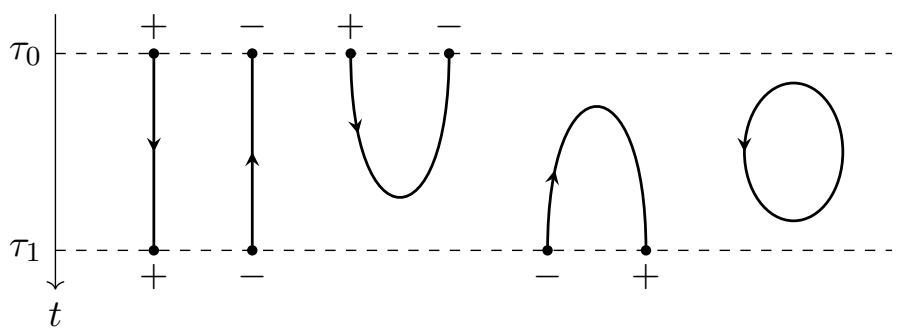

Figure 5. All possible connected oriented bordisms. We call them positively and negatively oriented intervals, left elbow, right elbow, and circle, respectively. The cut functions are $\rho_{i}=t-\tau_{i}$, so these pictures are read from top to bottom.

A field theory $Z \in 1$-TFT ${ }^{\text {or }}$ will assign vector spaces $V^{ \pm}$to the two different points. The elbow then provides a pairing $V^{+} \otimes V^{-} \rightarrow \mathbb{C}$, which must be non-degenerate due to the snake identity; hence $V^{-}$can be identified with the dual space of $V^{+}$and vice versa. In particular, we do not obtain the additional datum of a nondegenerate bilinear form on our vector spaces.

Passing from ordinary field theories to oriented field theories over a target $M$, the result is the following.

Theorem 4.24 (classification of 1-TFTs, oriented case). There is an equivalence of groupoids

$$
1-\operatorname{TFT}^{\text {or }}(M) \cong \operatorname{Vect}_{\nabla}(M),
$$

which is natural in $M$.

Here $\operatorname{Vect}_{\widetilde{\nabla}}(M)$ denotes the groupoid of vector bundles with connection on $M$, with morphisms given by connection-preserving bundle isomorphisms. The proof is analogous to that of Theorem 4.2.

\section{$5 \quad$ Field theories with values in sheaves}

In this section, we consider notions of "families of vector spaces" more general than vector bundles. Let $\mathcal{V}$ denote some stack of $C^{\infty}$-modules. This means that for each manifold $S$, objects of $\mathcal{V}(S)$ are modules over $C^{\infty}(S)$, possibly with a bornology or topology of a particular kind. Below, we will fix suitable conditions $\mathcal{V}$ should satisfy, but we would like, at a minimum, that the operation of taking global sections determines an embedding Vect $\rightarrow \mathcal{V}$, and a symmetric monoidal structure on $\mathcal{V}$ compatible with this embedding. In line with Definition 3.36, we write

$$
1-\operatorname{TFT}(M ; \mathcal{V})=\operatorname{Fun}^{\otimes}(1-\operatorname{Bord}(M), \mathcal{V})
$$


for the groupoid of 1-dimensional field theories over $M$ taking values in $\mathcal{V}$, and similarly for the oriented variant. In the TFT case, it is expected that this introduces no new examples, that is, a field theory with values in $\mathcal{V}$ automatically takes values in Vect. In this section, we verify that this is indeed the case.

Definition 5.1. We will call a symmetric monoidal smooth stack $\mathcal{V}$ an admissible stack of $C^{\infty}$-modules if the following holds.

(C1) For each $S, \mathcal{V}(S)$ is an additive category with kernels, naturally in $S$, such that $\otimes$ is additive in both variables.

(C2) There is a linear symmetric monoidal fibered functor Vect $\rightarrow \mathcal{V}$ which determines, for each $S$, an equivalence between $\operatorname{Vect}(S)$ and the dualizable objects of $\mathcal{V}(S)$.

(C3) Let $V \in \mathcal{V}(S \times \mathbb{R}), W \in \mathcal{V}(S)$, and pr: $S \times \mathbb{R} \rightarrow S$ be the projection. If $\sigma: V \rightarrow$ pr* $^{*}$ is such that $\left.\sigma\right|_{U}=0$ for every open $U \subset S \times \mathbb{R}$ with $U \cap(S \times\{0\})=\varnothing$, then $\sigma=0$.

Condition (C3) is a kind of separation axiom. It is often useful to regard a morphism $W^{\prime} \rightarrow W$ in $\mathcal{V}(S)$ as a generalized element of $W$. Then a morphism $\sigma: V \rightarrow \operatorname{pr}^{*} W$ as above can be interpreted as a "generalized path of elements" of $W$, and the axiom says that a generalized path vanishes altogether provided it vanishes on $\mathbb{R} \backslash 0$.

In Section 5.2, we give examples of admissible stacks of $C^{\infty}$-modules. Before doing this, we state and prove the following theorem, which contains the previously mentioned result that the target does not matter for topological field theories.

Theorem 5.2. For any admissible stack of $C^{\infty}$-modules $\mathcal{V}$ and any manifold $M$, the inclusions

$$
1-\operatorname{TFT}^{\mathrm{or}}(M) \rightarrow 1-\mathrm{TFT}^{\mathrm{or}}(M ; \mathcal{V}), \quad 1-\mathrm{TFT}(M) \rightarrow 1-\mathrm{TFT}(M ; \mathcal{V})
$$

are equivalences of groupoids.

Remark 5.3. Combining the above with Theorems 4.2 and 4.24 gives us equivalences of groupoids

$$
1-\operatorname{TFT}(M ; \mathcal{V}) \cong \operatorname{Vect}_{\nabla, \beta}(M), \quad 1-\operatorname{TFT}^{\text {or }}(M ; \mathcal{V}) \cong \operatorname{Vect}_{\nabla}(M)
$$

for any admissible $\mathcal{V}$. This is the exact meaning we intend for Theorems 1.1 and 1.2 , stated in less detail in the introduction.

\subsection{Proof of Theorem 5.2}

We will focus on the unoriented case, the oriented one being similar. Throughout, we fix a field theory $Z: 1$-Bord $(M) \rightarrow \mathcal{V}$, which we assume to be strict as a morphism of simplicial stacks.

Lemma 5.4. Let $c: M \times \mathbb{R} \rightarrow M$ be the tautological $M$-family of constant paths. Then the object $Z(c ; 0) \in \mathcal{V}$ is dualizable.

Proof. For $\gamma: S \times \mathbb{R} \rightarrow M$ and $a<b$, we denote by $L_{\gamma ; a, b}$ the left elbow given by this data, that is, the $S$-family of bordisms with underlying map $\gamma$ and cut functions $\rho_{0}(s, t)=(t-a)(t-b)$ and $\rho_{1}(s, t)=-\frac{1}{4}(b-a)^{2}$, where $t$ is the coordinate of $\mathbb{R}$ (here any $\rho_{1}$ can be chosen such that it has no zeroes, and such that $\rho_{1} \leq \rho_{0}$ everywhere). We write similarly $R_{\gamma ; a, b}$ for the right elbow. To prove the lemma, we consider in particular $S=M$ and $\gamma=c$; then for each $\delta>0$, the images of $L_{c ; 0, \delta}$ and $R_{c ; 0, \delta}$ are canonically identified as maps

$$
\begin{aligned}
& Z\left(L_{c ; 0, \delta}\right): Z(c ; 0) \otimes Z(c ; 0) \rightarrow \epsilon_{M}, \\
& Z\left(R_{c ; 0, \delta}\right): \epsilon_{M} \rightarrow Z(c ; 0) \otimes Z(c ; 0),
\end{aligned}
$$


where $\epsilon_{M}$ is the monoidal unit of $\mathcal{V}(M)$. We claim that these morphisms are independent of $\delta$. To see this, pick any diffeomorphism $\chi: \mathbb{R} \rightarrow \mathbb{R}$ with $\chi(0)=0, \chi(1)=\delta$ which is affine of slope 1 near 0 and 1. Then $\chi$ determines a morphism in 1 -Bord $(M)_{1}$ between $L_{c ; 0, \delta}$ and $L_{c \circ \chi ; 0,1}=L_{c ; 0,1}$, compatible with the usual identifications of their boundary components with $(c ; 0)$. This proves the claim.

Write $L=Z\left(L_{c ; 0, \delta}\right), R=Z\left(R_{c ; 0, \delta}\right)$ for this common value. Then, expressing the interval bordism $(c, 0, \delta)$ as a suitable composition of $L_{c ; 0, \delta}$ and $R_{c ; 0, \delta}$, we get

$$
(\mathrm{id} \otimes L) \circ(R \otimes \mathrm{id})=Z(c ; 0, \delta)
$$

for any $\delta>0$. Now consider, for instance, $\left(c, 0, \delta^{2}\right)$ as an $(M \times \mathbb{R})$-family of intervals, where $\delta$ now denotes the coordinate on the factor $\mathbb{R}$. Its image under $Z$ is a family of endomorphisms of $Z(c ; 0)$ that is constant away from $\delta=0$, and equal to the identity at $\delta=0$. Thus, from the separation axiom of $\mathcal{V}, Z(c ; 0, \delta)=Z(c ; 0,0)=\mathrm{id}_{Z(c ; 0)}$ for all $\delta$. This proves that $L$ and $R$ provide the desired evaluation and coevaluation maps.

Recall our notation $\operatorname{Path}_{c}(M) \subset$ 1-Bord $(M)$ for the subcategory of paths with sitting instants (Definition 4.8). Using the above lemma and axiom $(\mathrm{C} 2)$ for $\mathcal{V}$, we find a factorization of $\left.Z\right|_{\operatorname{Path}_{c}(M)}$ through Vect $\hookrightarrow \mathcal{V}$ (essentially uniquely). Proposition 4.16 then gives us a vector bundle with connection $(V, \nabla)$ on $M$ and an isomorphism

$$
\left.Z\right|_{\operatorname{Path}_{c}(M)} \cong Z V,\left.\nabla\right|_{\operatorname{Path}_{c}(M)}
$$

of smooth functors, where $Z_{V, \nabla}$ denotes the field theory determined by parallel transport on $V$. We will eventually show that $Z \cong Z_{V, \nabla, \beta}$ for some pairing $\beta$.

Lemma 4.22 is easily restated for families of bordisms and reproved for $\mathcal{V}$-valued field theories, using the separation axiom. We now adapt some other definitions and lemmas from Section 4 for which special care with the family aspect is crucial. Let $\gamma: S \times \mathbb{R} \rightarrow M$ be an $S$-family of paths and let $a: S \rightarrow \mathbb{R}$ be any smooth function, so that we can talk about the $S$-family of objects $(\gamma ; a)$ (where its cut function is $\rho_{a}(s, t)=t-a(s)$ ). Note that by the implicit function theorem, any cut function $\rho$ on $S \times \mathbb{R}$ is equivalent to some $\rho_{a}$ : Just take $a$ defined by $\rho(s, a(s))=0$. Similarly, two functions $a \leq b: S \rightarrow \mathbb{R}$ determine a family of intervals $(\gamma ; a, b)$. These examples exhaust all possible isomorphism classes of objects, respectively bordisms.

Definition 5.5. Given functions $a, b: S \rightarrow \mathbb{R}$ with $a<b$, a left modification function is a smooth map $\chi: S \times \mathbb{R} \rightarrow S \times \mathbb{R}$ of the form $\chi(s, t)=(s, \tilde{\chi}(s, t))$ for some $\tilde{\chi}: S \times \mathbb{R} \rightarrow \mathbb{R}$ such that

(1) for each $s \in S, t \mapsto \tilde{\chi}(s, t)$ is nondecreasing,

(2) there exists a neighborhood of $\{t=b\} \subset S \times \mathbb{R}$ where $\chi$ equals the identity,

(3) there exists a neighborhood of $\{t=a\}$, where $\tilde{\chi}(s, t)=a(s)$.

We define right modification functions by swapping the roles of $a$ and $b$, and two-sided modification functions by requiring fiberwise constancy near both ends.

Lemma 5.6. For any $a>b: S \times \mathbb{R} \rightarrow \mathbb{R}$, there exist left, right and two-sided modification functions on $S \times \mathbb{R}$.

Proof. We can certainly find a nonnegative smooth function $f: S \times \mathbb{R} \rightarrow \mathbb{R}$ with support in the open set $\{(s, t) \mid a(s)<t<b(s)\} \subset S \times \mathbb{R}$ and such that $\left.f\right|_{\{s\} \times \mathbb{R}}$ is not identically zero for any $s \in S$. Setting

$$
g(s, t)=\frac{\int_{-\infty}^{t} f(s, u) \mathrm{d} u}{\int_{-\infty}^{+\infty} f(s, u) \mathrm{d} u}
$$


gives us a smooth, fiberwise nondecreasing function which is identically 0 in a neighborhood of $\{t \leq a\}$ and identically 1 in a neighborhood of $\{t \geq b\}$. Then

$$
\chi_{L R}(s, t)=(s, a(s)+(b(s)-a(s)) g(s, t))
$$

is a two-sided modification function. Next, write

$$
\kappa(s)=\frac{1}{\int_{-\infty}^{+\infty} f(s, u) \mathrm{d} u}\left(b(s)-a(s)-\int_{-\infty}^{b(s)} g(s, u) \mathrm{d} u\right) .
$$

Due to the bounds on $g$, this is nonnegative. Finally, set

$$
h_{s}(t)=a(s)+\int_{-\infty}^{t}(\kappa(s) f(s, u)+g(s, u)) \mathrm{d} u .
$$

Clearly, $h_{s}$ is nondecreasing, constant near $t=a(s)$, and affine with slope 1 near $t=b(s)$. Moreover, by the choice of $\kappa(s)$, we have $h_{s}(a(s))=a(s)$ and $h_{s}(b(s))=b(s)$. Thus,

$$
\chi_{L}(s, t)=\left(s, h_{s}(t)\right)
$$

defines a left modification function. Right modification functions can be constructed similarly.

Lemma 5.7. Let $\gamma: S \times \mathbb{R} \rightarrow M$ be an $S$-family of paths, fix smooth functions $a<b: S \rightarrow \mathbb{R}$, and let $\chi_{0}, \chi_{1}$ be left modification functions. Then $Z\left(\gamma \circ \chi_{0} ; a, b\right)=Z\left(\gamma \circ \chi_{1} ; a, b\right)$. The same holds for right modifications and two-sided modifications.

Note that all modification functions agree in a neighborhood of the boundaries, so the domain and codomain of the maps $Z(\gamma \circ \chi, a, b)$ are canonically identified, as in Remark 4.15.

Proof. The case of two-sided modifications follows immediately from Lemma 4.23, since Vect is full in $\mathcal{V}$, so that the maps $Z\left(\gamma \circ \chi_{i}, a, b\right)$ are identified with maps of vector bundles. Suppose now that $\chi_{0}, \chi_{1}$ are left modification functions. Let $U$ be a neighborhood of $\{t=b\}$, where $\chi_{0}$ and $\chi_{1}$ both agree with the identity. Then we can find a function $a^{\prime}$ with $a>a^{\prime}>b$ and $\left\{t=a^{\prime}\right\} \subset U$, as well as a map $F: S \times \mathbb{R} \rightarrow S \times \mathbb{R}$ over $S$, fiberwise nondecreasing, which is the identity away from $U$ and near $\{b=0\}$, and fiberwise constant on $\left\{t=a^{\prime}\right\}$. Thus, using Lemma 4.22, we get

$$
Z\left(\gamma \circ \chi_{i}, a, b\right)=Z\left(\gamma \circ \chi_{i} \circ F, a, b\right)=Z\left(\gamma \circ F, a^{\prime}, b\right) \circ Z\left(\gamma \circ \chi_{i} \circ F, a, a^{\prime}\right)
$$

for both $i=0,1$. Finally, notice that

$$
Z\left(\gamma \circ \chi_{0} \circ F, a, a^{\prime}\right)=Z\left(\gamma \circ \chi_{1} \circ F, a, a^{\prime}\right)
$$

by the two-sided version of this lemma. This finishes the proof of the left-sided case; the rightsided case is similar.

Lemma 5.8. The field theory $Z$ determines a stack map $Z^{\prime}: 1$-Bord $(M)_{0} \rightarrow \mathcal{V}$ and a distinguished decomposition

$$
Z(\gamma ; a)=V_{\gamma(a)} \oplus Z^{\prime}(\gamma ; a)
$$

for each $S$-family of objects $(\gamma, a)$, where $V_{\gamma(a)}$ is the pullback of the vector bundle $V \rightarrow M$ via the map $\gamma(a): S \rightarrow M$. 
Above, we used the suggestive notation $\gamma(a)$ for the map $s \mapsto \gamma(s, a(s))$. Note that $V_{\gamma(a)} \cong$ $Z(\gamma(a), a)$ is what $Z$ assigns to the family of constant germs with the same value as $\gamma$ on $\{t=a\}$.

Proof. Choose smooth functions $a^{\prime}, a^{\prime \prime}: S \rightarrow \mathbb{R}$ with $a^{\prime}<a<a^{\prime \prime}$ and smooth, fiberwise nondecreasing functions $\chi_{1}, \chi_{2}: S \times \mathbb{R} \rightarrow S \times \mathbb{R}$ such that $\chi_{1}$ is fiberwise constant near $a^{\prime}$ and $a^{\prime \prime}$ and equal to the identity near $a$, while $\chi_{2}$ is fiberwise constant near $a^{\prime}, a$ and $a^{\prime \prime}$. In other words, $\chi_{1}$ is a left modification function for $\left(\gamma ; a^{\prime}, a\right)$ and a right modification function for $\left(\gamma ; a, a^{\prime \prime}\right)$, while $\chi_{2}$ is a two-sided modification function for both $\left(\gamma ; a^{\prime}, a\right)$ and $\left(\gamma ; a, a^{\prime \prime}\right)$. In particular, both $\chi_{1}$ and $\chi_{2}$ are two-sided modification functions for $\left(\gamma ; a^{\prime}, a^{\prime \prime}\right)$. The existence of such functions is clear from Lemma 5.6 and the fact that modification functions can be "patched together" in the obvious way for neighboring intervals. We obtain the following diagram of bordisms:

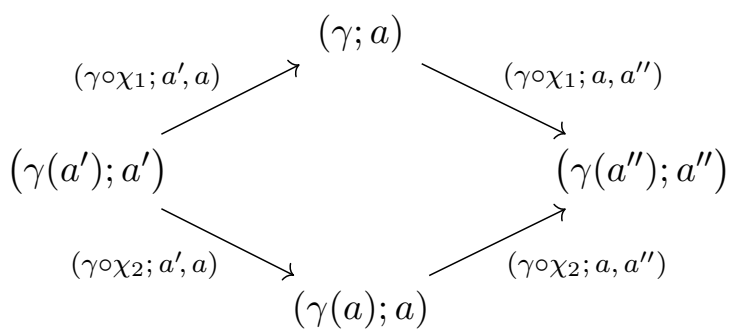

It certainly does not commute in the sense of there being an isomorphism between the two compositions: the bottom one has a sitting instant at $a$, while in general the top one does not. However, since the composition of the upper two bordisms is $\left(\gamma \circ \chi_{1} ; a^{\prime}, a^{\prime \prime}\right)$ while the lower composition is $\left(\gamma \circ \chi_{2} ; a^{\prime}, a^{\prime \prime}\right)$, Lemma 5.7 implies that the diagram obtained after applying $Z$

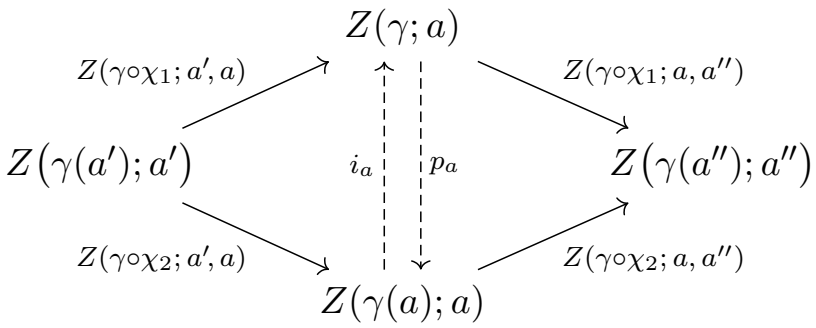

commutes. We now define maps $i_{a}$ and $p_{a}$ as indicated in the diagram by requiring that they make the left, respectively right, triangle commute. These maps exist and are well-defined as the bottom maps are invertible, by Lemma 4.17. By commutativity of the diagram, we have $p_{a} i_{a}=\mathrm{id}$.

We now set $Z^{\prime}(\gamma ; a)=\operatorname{Ker} p_{a}$. By standard arguments, $i_{a}$ and the inclusion map $Z^{\prime}(\gamma ; a) \rightarrow$ $Z(\gamma ; a)$ exhibit $Z(\gamma ; a)$ as the direct sum

$$
Z(\gamma ; a) \cong V_{\gamma(a)} \oplus Z^{\prime}(\gamma ; a)
$$

This identification does not depend on any of the choices by Lemma 5.7.

Lemma 5.9. For any nowhere thin S-family of bordisms $(\gamma ; a, b)$, by which we mean that $a(s)<b(s)$ for all $s \in S$, the map $Z(\gamma ; a, b)$ has matrix representation

$$
Z(\gamma ; a, b)=\left(\begin{array}{cc}
P(\gamma ; a, b) & 0 \\
0 & 0
\end{array}\right)
$$

with respect to the direct sum decompositions of $Z(\gamma ; a)$ and $Z(\gamma ; b)$ from Lemma 5.8. 
Proof. Choose $a^{\prime}<a<a^{\prime \prime}<b^{\prime}<b<b^{\prime \prime}$ (as functions $S \rightarrow \mathbb{R}$ ) and consider the commutative diagram

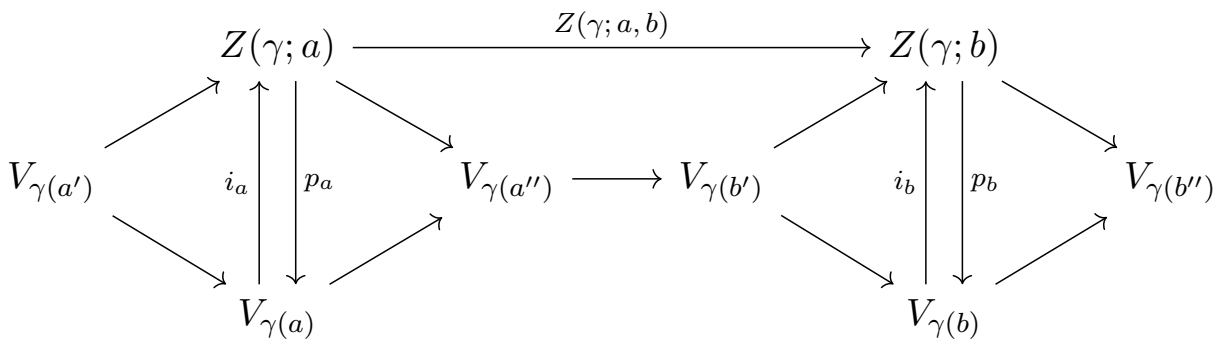

obtained by patching together the corresponding diagrams (5.1) in the obvious way. In particular, all bottom maps are parallel translations along segments of $\gamma$.

To prove that the second columns of the matrix representing $Z(\gamma ; a, b)$ is zero, we need to show that this map, restricted to the kernel of $p_{a}$, is zero. This follows from the diagram, since it shows that $Z(\gamma ; a, b)$ factors through $p_{a}$.

A diagram chase shows that the square composed by $Z(\gamma ; a, b), i_{a}, i_{b}$, and the parallel translation $P: V_{\gamma(a)} \rightarrow V_{\gamma(b)}$ commutes. This implies that the first column of $Z(\gamma ; a, b)$ is as claimed.

The proof of Theorem 5.2 concludes with the next lemma.

Lemma 5.10. For any $S$-family $(\gamma ; a), Z^{\prime}(\gamma ; a)=0 \in \mathcal{V}(S)$.

Proof. Let pr: $S \times \mathbb{R} \rightarrow S$ be the projection and let $\delta: S \times \mathbb{R} \rightarrow \mathbb{R}$ be the coordinate function on the $\mathbb{R}$-factor. Consider the $(S \times \mathbb{R})$-family of bordisms $B=\left((\operatorname{pr} \times \mathrm{id})^{*} \gamma ; \operatorname{pr}^{*} a-\delta^{2}, \operatorname{pr}^{*} a\right)$. Then

$$
Z^{\prime}(B): Z^{\prime}\left(\operatorname{pr}^{*} \gamma, a-\delta^{2}\right) \rightarrow Z^{\prime}\left(\operatorname{pr}^{*} \gamma, a\right) \cong \operatorname{pr}^{*}\left(Z^{\prime}(\gamma, a)\right)
$$

vanishes away from $S \times 0$ by the previous lemma, and therefore is identically 0 by the separation axiom. But this implies that

$$
\operatorname{id}_{Z^{\prime}(\gamma ; a)}=\left.Z^{\prime}(B)\right|_{S \times 0}=0,
$$

so $Z^{\prime}(\gamma ; a)$ is the zero object of $\mathcal{V}(S)$.

\subsection{Examples of admissible stacks of $C^{\infty}$-modules}

In this section, we give two examples of suitable target stacks $\mathcal{V}$ for TFTs:

1. The stack $\mathcal{V}_{\text {alg }}$ of sheaves of $C^{\infty}$-modules with the algebraic tensor product.

2. The stack $\mathcal{V}_{\mathrm{vN}}$ of sheaves of complete bornological $C^{\infty}$-modules with the completed bornological tensor product, constructed in Appendix A.5 and briefly reviewed below. Here, $C^{\infty}(S)$ is endowed with its von Neumann bornology (see Example A.2), hence the notation.

Recall that a bornology on a vector space is a collection of subsets deemed to be bounded and satisfying appropriate axioms. Bornological vector spaces and bounded linear maps form a category Born. There is an appropriate notion of completeness, and thus a full subcategory CBorn $\subset$ Born of complete bornological vector spaces. We are interested in CBorn for its pleasant categorical properties. In particular, there is a completed tensor product $\hat{\otimes}$ which makes CBorn into a closed symmetric monoidal category. As is well known, none of the many possible tensor products on the various categories of topological vector spaces have this property. See Appendices A.1 to A.3 for a quick overview, or Meyer [8, Chapter 1] for a comprehensive introduction to the theory of bornological vector spaces.

The goal of this subsection, which will follow immediately by combining Propositions 5.17 and 5.19 and Corollaries 5.18 and 5.20 below, is to prove the following. 
Theorem 5.11. $\mathcal{V}_{\mathrm{alg}}$ and $\mathcal{V}_{\mathrm{vN}}$ are admissible stacks of $C^{\infty}$-modules.

Remark 5.12. The purely algebraic $\mathcal{V}_{\text {alg }}$ is familiar and attractive in its simplicity, but it has the drawback of not admitting a reasonable "sheaf of sections" functor from any category of infinite-dimensional vector bundles. Moreover, our proof that it satisfies the separation axiom requires the corresponding fact for $\mathcal{V}_{\mathrm{vN}}$.

Before proceeding, let us briefly review the definitions of $\mathcal{V}_{\text {alg }}$ and $\mathcal{V}_{\mathrm{vN}}$. It will be convenient to use the sheaf-of-categories approach to stacks.

We define $\mathcal{V}_{\text {alg }}: \mathrm{Man}^{\mathrm{op}} \rightarrow \mathrm{Cat}^{\otimes}$ to be the functor assigning to each manifold $S$ the symmetric monoidal category of sheaves of $C_{S}^{\infty}$-modules and $C_{S}^{\infty}$-linear maps, with the algebraic tensor product. To a map $f: T \rightarrow S$ is associated the pullback functor $f^{*}$ defined by

$$
f^{*} V=C_{T}^{\infty} \otimes_{f^{-1}\left(C_{S}^{\infty}\right)} f^{-1}(V) .
$$

That this indeed defines a symmetric monoidal stack on the site of manifolds and satisfies axiom (C1) is a standard fact.

Similar constructions can be carried out in the setting of (complete) bornological vector spaces. This is outlined in Appendices A.4 and A.5 and is mostly a formality, except for the fact that sheafification of presheaves with values in CBorn requires additional care. In a nutshell, we promote the sheaf of smooth functions on a manifold $S$ to a sheaf of complete bornological algebras, denoted by $C_{\mathrm{vN}, S}^{\infty}$, and then define $\mathcal{V}_{\mathrm{vN}}(S)$ to be the category of sheaves of complete bornological $C_{\mathrm{vN}, S}^{\infty}$-modules. We have a completed tensor product of bornological sheaves, and the pullback functors $f^{*}: \mathcal{V}_{\mathrm{vN}}(S) \rightarrow \mathcal{V}_{\mathrm{vN}}(T)$ are defined similarly to (5.2), using this completed tensor product. Thus $\mathcal{V}_{\mathrm{vN}}$ is a symmetric monoidal stack satisfying axiom (C1).

Next, we construct a functor $i: \mathcal{V}_{\text {alg }} \rightarrow \mathcal{V}_{\mathrm{vN}}$. Let $S$ be a manifold and $V \in \mathcal{V}_{\text {alg }}(S)$. Given $U \subset S$ open, we say that a subset $B \subset V(U)$ is vN-bounded if each point $x \in U$ has an open neighborhood $W \subset U$ such that

$$
\left.B\right|_{W} \subset C_{1} b_{1}+\cdots+C_{n} b_{n}
$$

for vN-bounded subsets $C_{1}, \ldots, C_{n} \subset C^{\infty}(U)$ and $b_{1}, \ldots, b_{n} \in V(W)$. It is routine to check that this indeed satisfies the axioms of a (vector) bornology on $V(U)$. We denote the resulting presheaf of bornological vector spaces by $V_{\mathrm{vN}}$.

Example 5.13. If $V=\Gamma_{E}$ is the sheaf of sections of a finite-dimensional vector bundle over $E \rightarrow S$, then the bornology constructed above coincides with the bornology associated to its Fréchet topology, where a set of sections is bounded if all derivatives of a given order are uniformly bounded on compact sets.

Proposition 5.14. For any sheaf $V$ of $C_{S}^{\infty}$-modules, $V_{\mathrm{vN}}$ is a sheaf of complete bornological $C_{\mathrm{vN}, S}^{\infty}$-modules.

Proof. It follows directly from the definition of the vN-bornology that for each open $U \subseteq S$, the bornological vector space $V_{\mathrm{vN}}(U)$ is a bornological $C_{\mathrm{vN}}^{\infty}(U)$-module (i.e., the module action map is bounded), and that restriction maps are bounded. We have to show that $V_{\mathrm{vN}}$ satisfies descent. Let $U \subset S$ be open and let $\left\{U_{i}\right\}_{i \in I}$ be an open cover of $U$. Since $V$ is an (algebraic) sheaf, we know that the map

$$
V_{\mathrm{vN}}(U) \stackrel{r}{\longrightarrow} \operatorname{Ker}\left(\prod_{i \in I} V_{\mathrm{vN}}\left(U_{i}\right) \longrightarrow \prod_{i, j \in I} V_{\mathrm{vN}}\left(U_{i} \cap U_{j}\right)\right)
$$

is a vector space isomorphism. We have to show that it is an isomorphism of bornological vector spaces. Since we already know that restriction maps are bounded, we have to show that the 
inverse image under $r$ of bounded sets in $\prod_{i \in I} V_{\mathrm{vN}}\left(U_{i}\right)$ is bounded. It suffices to show this for sets of the form $\prod_{i \in I} B_{i}$ with $B_{i} \subset V_{\mathrm{vN}}\left(U_{i}\right)$ bounded, as these generate the product bornology. Here we have

$$
r^{-1}\left(\prod_{i \in I} B_{i}\right)=\left\{v \in V(U)|v|_{U_{i}} \in B_{i}\right\} .
$$

That this is vN-bounded follows again directly from the definition of the bornology, as boundedness can be checked locally. This shows that $V_{\mathrm{vN}}$ is a sheaf of bornological $C_{\mathrm{vN}, S}^{\infty}$-modules.

We now show that $V_{\mathrm{vN}}(U)$ is complete for each open $U \subseteq S$. To this end, we have to show that each bounded subset of $V_{\mathrm{vN}}(U)$ is contained in a complete bounded disc. Here a bounded disc is complete if the normed space $V(U)_{B}=\operatorname{span}(B) \subset V(U)$ is complete, where the norm is the Minkowski functional defined by $B$. Let $\left\{W_{i}\right\}_{i \in I}$ be an open cover of $U$, let $b_{1}^{i}, \ldots, b_{n_{i}}^{i} \in V\left(W_{i}\right)$ and $C_{1}^{i}, \ldots, C_{n_{i}}^{i} \subset C^{\infty}\left(W_{i}\right)$ be complete vN-bounded discs. Then the sets $B_{i}:=C_{1}^{i} b_{1}^{i}+\cdots+C_{n_{i}}^{i} b_{n_{i}}^{i} \subset V\left(W_{i}\right)$ and

$$
B:=\left\{v \in V(U)|v|_{W_{i}} \in B_{i}\right\}
$$

are vN-bounded discs. Clearly, any vN-bounded set in $V(U)$ is contained in such a disc, for some choice of $\left\{W_{i}\right\},\left\{b_{i}\right\}$ and $\left\{C_{k}^{i}\right\}$, hence it suffices to show that $V(U)_{B}$ is a Banach space.

We first show that each of the normed spaces $V\left(W_{i}\right)_{B_{i}}$ are complete. To this end, consider the linear map

$$
\pi: \quad X \longrightarrow V\left(W_{i}\right)_{B_{i}}, \quad X:=\prod_{k=1}^{n_{i}} C^{\infty}\left(W_{i}\right)_{C_{k}^{i}},
$$

sending $\left(f_{1}, \ldots, f_{n_{i}}\right)$ to $f_{1} b_{1}+\cdots+f_{n_{i}} b_{n_{i}}$. It is surjective by definition of $B_{i}$, and it is bounded since any bounded set $C \subset X$ is contained in $\lambda\left(C_{1}^{i} \times \cdots \times C_{n_{i}}^{i}\right)$ for some $\lambda>0$, hence $\pi(C) \subset$ $\lambda \pi\left(C_{1}^{i} \times \cdots \times C_{n_{i}}^{i}\right)=\lambda B$. Notice that $X$ is a Banach space as the discs $C_{k}^{i}$ are complete. Because $\pi$ is bounded, its kernel is closed, so that the quotient $X / \operatorname{Ker}(\pi)$ is a Banach space. The quotient map $\bar{\pi}: X / \operatorname{Ker}(\pi) \rightarrow V\left(W_{i}\right)$ is a bounded vector space isomorphism. To see that it is a homeomorphism, it remains to show that the inverse is bounded. To this end, let $v \in V\left(W_{i}\right)_{B_{i}}$. Let $\lambda>0$ be such that $v \in \lambda B_{i}$. Because $\pi\left(C_{1}^{i} \times \cdots \times C_{n_{i}}^{i}\right)=B_{i}$, we can choose a preimage $v^{\prime} \in \lambda\left(C_{1}^{i} \times \cdots \times C_{n_{i}}^{i}\right) \subset X$. Then

$$
\begin{aligned}
\left\|\bar{\pi}^{-1}(v)\right\|_{X / \operatorname{Ker}(\pi)} & =\inf _{w \in \operatorname{Ker}(\pi)}\left\|v^{\prime}+w\right\|_{X} \leq\left\|v^{\prime}\right\|_{X} \\
& =\inf \left\{\mu>0 \mid v^{\prime} \in \mu\left(C_{1}^{i} \times \cdots \times C_{n_{i}}^{i}\right)\right\} \leq \lambda .
\end{aligned}
$$

This holds for any $\lambda$ such that $v \in \lambda B_{i}$. Therefore, we get

$$
\left\|\bar{\pi}^{-1}(v)\right\|_{X / \operatorname{Ker}(\pi)} \leq \inf \left\{\lambda>0 \mid v \in \lambda B_{i}\right\}=\|v\|_{B_{i}} .
$$

This shows that $\bar{\pi}^{-1}$ is bounded, hence $\bar{\pi}: X / \operatorname{Ker}(\pi) \rightarrow V\left(W_{i}\right)_{B_{i}}$ is a homeomorphism. Since $X / \operatorname{Ker}(\pi)$ is complete, so is $V\left(W_{i}\right)_{B_{i}}$.

Now consider the map

$$
V(U)_{B} \stackrel{r}{\longrightarrow} \prod_{i \in I} V\left(W_{i}\right)_{B_{i}}, \quad v \mapsto\left(\left.v\right|_{W_{i}}\right)_{i \in I},
$$

where the right hand side is a product of Banach spaces, endowed with the norm obtained by taking the supremum over all the norms of the $V\left(W_{i}\right)_{B_{i}}$. In other words, the unit ball of the product is $\prod_{i \in I} B_{i}$. Observe that this map is well-defined since if $v \in \lambda B$, then $\left.v\right|_{W_{i}} \in \lambda B_{i}$ 
and $\left(\left.v\right|_{W_{i}}\right) \in \lambda \prod_{i \in I} B_{i}$. The map is bornologically proper, since $r^{-1}\left(\prod_{i \in I} B_{i}\right)=B$. On the other hand, its image is the kernel of the bounded map

$$
\prod_{i \in I} V\left(W_{i}\right)_{B_{i}} \longrightarrow \prod_{i, j \in I} V\left(W_{i} \cap W_{j}\right)_{B_{i j}}, \quad\left(v_{i}\right)_{i \in I} \longmapsto\left(\left.v_{i}\right|_{W_{i} \cap W_{j}}-\left.v_{j}\right|_{W_{i} \cap W_{j}}\right)_{i, j \in I}
$$

hence closed. Here $B_{i j}=\left.B_{i}\right|_{W_{i} \cap W_{j}}+\left.B_{j}\right|_{W_{i} \cap W_{j}}$. This shows that $V(U)_{B}$ is isomorphic to a closed subspace of a Banach space, hence complete.

To promote the assignment $V \mapsto V_{\mathrm{vN}}$ to a functor, we have to deal with morphisms. Here we have the following lemma.

Lemma 5.15. Let $V$ and $V^{\prime}$ be (algebraic) $C_{S}^{\infty}$-modules and let $\Phi: V \rightarrow V^{\prime}$ be a morphism of sheaves. Then, for each $U \subseteq S, \Phi_{U}: V(U) \rightarrow V^{\prime}(U)$ is vN-bounded.

Proof. Let $B \subset V(U)$ be vN-bounded. We have to show that also $\Phi_{U}(B)$ is vN-bounded. To this end let $x \in U$ and choose $W$ such that $\left.B\right|_{W} \subseteq C_{1} b_{1}+\cdots+C_{n} b_{n}$ for elements $b_{1}, \ldots, b_{n}$ $\in V(W)$ and vN-bounded subsets $C_{1}, \ldots, C_{n} \subset C^{\infty}(W)$. Then since $\Phi$ is a morphism of sheaves and $C_{S}^{\infty}$-linear,

$$
\Phi_{U}(B)=\Phi_{W}\left(\left.B\right|_{W}\right) \subseteq \Phi\left(C_{1} b_{1}+\cdots+C_{n} b_{n}\right)=C_{1} \Phi\left(b_{1}\right)+\cdots+C_{n} \Phi\left(b_{n}\right) .
$$

Hence $\Phi_{U}(B)$ is vN-bounded.

We therefore obtain a functor $i: \mathcal{V}_{\text {alg }} \rightarrow \mathcal{V}_{\mathrm{vN}}$ sending $V$ to $V_{\mathrm{vN}}$ and which sends morphisms $V \rightarrow V^{\prime}$ of sheaves to the corresponding morphism $V_{\mathrm{vN}} \rightarrow V_{\mathrm{vN}}^{\prime}$, which exists by the previous lemma. Since conversely, each morphism of sheaves $V_{\mathrm{vN}} \rightarrow V_{\mathrm{vN}}^{\prime}$ gives a morphism of the underlying algebraic sheaves of $C_{S}^{\infty}$-modules, we obtain the following corollary.

Corollary 5.16. The functor $i: \mathcal{V}_{\mathrm{alg}} \rightarrow \mathcal{V}_{\mathrm{vN}}$ described above is fully faithful.

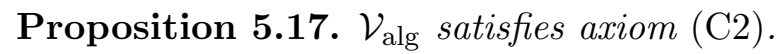

Proof. For each $S$, there is a "sheaf of sections" functor $\Gamma: \operatorname{Vect}(S) \rightarrow \mathcal{V}_{\text {alg }}(S)$. To see that these fit together to a map of stacks, we need to show that for each vector bundle $V \in \operatorname{Vect}(S)$ and each map $f: T \rightarrow S$, the natural maps $f^{*}\left(\Gamma_{V}\right) \rightarrow \Gamma_{f^{*} V}$ are isomorphisms. On the stalk at $x \in T$, this yields a map

$$
f^{*}\left(\Gamma_{V}\right)_{x} \cong C_{T, x}^{\infty} \otimes_{C_{S, f(x)}^{\infty}} \Gamma_{V, f(x)} \rightarrow \Gamma_{f^{*} V, x}
$$

compatible with the identifications $\Gamma_{V, f(x)} \cong C_{S, f(x)}^{\infty} \otimes V_{f(x)}$ and $\Gamma_{f^{*} V, x} \cong C_{T, x}^{\infty} \otimes V_{f(x)}$. Here, $V_{f(x)}$ is the fiber of the vector bundle $V$; all other subscripts denote stalks at the given point. Thus, the above is an isomorphism on stalks and hence an isomorphism of sheaves. That $\Gamma$ has all dualizable objects as essential image is the content of the Serre-Swan theorem.

Corollary 5.18. $\mathcal{V}_{\mathrm{vN}}$ satisfies axiom $(\mathrm{C} 2)$.

Proof. Composition with $i$ gives the map $\Gamma$ : Vect $\rightarrow \mathcal{V}_{\mathrm{vN}}$. Since every dualizable $C_{\mathrm{vN}, S^{-m o d u l e}}^{\infty}$ has finite rank, any dualizable object in $\mathcal{V}_{\mathrm{vN}}(S)$ is already dualizable in $\mathcal{V}_{\text {alg }}(S)$.

Proposition 5.19. $\mathcal{V}_{\mathrm{vN}}$ satisfies the separation axiom (C3). 
Proof. Fix a $C_{\mathrm{vN}, S}^{\infty}$-module $W$, and denote by $V$ its pullback to $S \times \mathbb{R}$. It suffices to show that if a section $\sigma \in V(U)$ is such that $\left.\sigma\right|_{U \backslash(S \times 0)}$ vanishes, then in fact $\sigma=0$. By definition, $V$ is the sheafification of the presheaf

$$
V^{\prime}: \quad U \mapsto C_{\mathrm{vN}}^{\infty}(U) \hat{\otimes}_{\left(\mathrm{pr}^{-1} C_{\mathrm{vN}, S}^{\infty}\right)(U)}\left(\mathrm{pr}^{-1} W\right)(U),
$$

so we know that $\sigma$ is determined by a coherent collection of sections $\sigma_{i} \in V^{\prime}\left(S_{i} \times T_{i}\right)$ for some collection of opens $S_{i} \subset S, T_{i} \subset \mathbb{R}$, since products $S_{i} \times T_{i}$ form a basis for the topology of $U \subset S \times \mathbb{R}$. Our question is reduced to showing that if $\sigma_{i}$ vanishes on $S_{i} \times\left(T_{i} \backslash 0\right)$, then it vanishes on $S_{i} \times T_{i}$. Now,

$$
\begin{aligned}
V^{\prime}\left(S_{i} \times T_{i}\right) & \cong C_{\mathrm{vN}}^{\infty}\left(T_{i} \times S_{i}\right) \hat{\otimes}_{C_{\mathrm{vN}}^{\infty}\left(S_{i}\right)} V\left(S_{i}\right) \\
& \cong\left(C^{\infty}\left(T_{i}\right) \hat{\otimes} C^{\infty}\left(S_{i}\right)\right) \hat{\otimes}_{C^{\infty}\left(S_{i}\right)} V\left(S_{i}\right) \\
& \cong C_{\mathrm{vN}}^{\infty}\left(T_{i}\right) \hat{\otimes} V\left(S_{i}\right) \\
& \cong C_{\mathrm{vN}}^{\infty}\left(T_{i} ; V\left(S_{i}\right)\right) .
\end{aligned}
$$

The first identification is by definition, the second follows from Proposition A.14, the third from the fact that $C^{\infty}\left(T_{i}\right) \hat{\otimes}$ - commutes with the colimit defining the tensor product over $C^{\infty}\left(S_{i}\right)$, and the fourth from Proposition A.13. Thus, $\sigma_{i}$ gets identified with a smooth function $f: T_{i} \rightarrow V\left(S_{i}\right)$ which, by definition, is a smooth function with values in some Banach space $V_{B} \subset V\left(S_{i}\right)$ (cf. Appendix A.3). Hence it vanishes identically if it vanishes away from 0 .

Corollary 5.20. $\mathcal{V}_{\text {alg }}$ satisfies the separation axiom (C3).

Remark 5.21. One might wish for a more elementary proof of the corollary, and we would like to note that the bornological tensor product is handy in this case as well. Arguing as in the proof of Proposition 5.19, one reduces property (C3) for $\mathcal{V}_{\text {alg }}$ to the claim that if $V$ is a $C^{\infty}(S)$-module and

$$
\sigma \in C^{\infty}(\mathbb{R} \times S) \otimes_{C^{\infty}(S)} V
$$

maps to 0 in $C^{\infty}((\mathbb{R} \backslash 0) \times S) \otimes_{C^{\infty}(S)} V$, then $\sigma=0$. This is not immediately clear. Notice, however, that if we give $V$ the fine bornology, Proposition A.11 allows us to regard $\sigma$ as an element of

$$
C_{\mathrm{vN}}^{\infty}(\mathbb{R} \times S) \hat{\otimes}_{C^{\infty}(S)} V \cong C_{\mathrm{vN}}^{\infty}(\mathbb{R}) \hat{\otimes} C_{\mathrm{vN}}^{\infty}(S) \hat{\otimes}_{C^{\infty}(S)} V
$$

and therefore as a smooth function from $\mathbb{R}$ to some Banach space. The claim follows.

\section{A Bornological sheaves}

In this appendix, we construct the symmetric monoidal stack $\mathcal{V}_{\mathrm{vN}}$ of sheaves of complete bornological $C^{\infty}$-modules on the site Man of smooth manifolds. Formally, this construction is very similar to that of its well-known algebraic counterpart $\mathcal{V}_{\text {alg }}$, and our main goal here is to highlight the differences. We start recalling several basic facts about bornological vector spaces, providing proofs for the bits that are not easily located in the literature. For this, our main reference is Meyer [8, Chapter 1]. The basic facts about sheaves of (complete) bornological vector spaces and modules are quoted from Houzel [5]. 


\section{A.1 Bornological vector spaces}

A (convex) bornology on a vector space $V$ over $\mathbb{K}=\mathbb{R}$ or $\mathbb{C}$ is a collection $\mathfrak{S}$ of subsets of $V$ deemed to be bounded. These have to satisfy appropriate axioms which we will not repeat here. For any collection $\mathfrak{S}^{\prime}$ of subsets of $V$, there is a smallest bornology containing $\mathfrak{S}^{\prime}$, the bornology generated by $\mathfrak{S}^{\prime}$. A linear map $f: V \rightarrow W$ is bounded if it sends bounded subsets to bounded subsets.

A disk in a vector space $V$ is a convex, balanced subset $B \subset V$. We denote by $V_{B}=\mathbb{R} \cdot B \subset V$ the subspace spanned by $B$. The closed ball of a seminorm on $V_{B}$ is an absorbing disk, and conversely a disk $B \subset V$ determines a unique seminorm on $V_{B}$. We say that $B$ is norming respectively complete if $V_{B}$ is a normed respectively a Banach space. A bornological vector space is separated if every bounded disk is norming, and complete if every bounded subset is contained in a complete bounded disk.

Example A.1. The smallest possible bornology on a vector space $V$ is the one generated by convex hulls of finite subsets. This is called the fine bornology. It is always complete. We denote by $\operatorname{Fin}(V)$ this bornological vector space. With this bornology, we have the relation $V \cong$ colim $V_{\alpha}$, where $V_{\alpha}$ runs through all finite-dimensional subspaces of $V$, endowed with their fine bornology.

Example A.2. Let $V$ be a locally convex topological vector space. Traditionally, a subset of $B \subset V$ is called bounded if it is absorbed by any neighborhood of the origin. This defines a bornology on $V$, called the von Neumann bornology. It is complete if $V$ is complete as a topological vector space. We denote by $\mathrm{vN}(V)$ this bornological vector space.

We are interested in bornological vector spaces for their convenient categorical properties. We denote by

$$
\text { CBorn } \subset \text { Born }
$$

the category of bornological vector spaces and its full subcategory of complete bornological vector spaces. ${ }^{1}$

Theorem A.3 ([8, Proposition 1.126]). Born and CBorn are additive categories and admit all limits and colimits.

The forgetful functor Born $\rightarrow$ Vect preserves limits and colimits; in other words, limits and colimits in Born are obtained by putting appropriate bornologies on the corresponding constructions with plain vector spaces. The bornology on a direct sum $\bigoplus_{i \in I} V_{i}$ is generated by images of bounded subsets of each $V_{i}$ via the standard inclusions; a subset $B \subset \prod_{i \in I} V_{i}$ is bounded if and only if each projection $p_{i}(B) \subset V_{i}$ is bounded; kernels and cokernels are endowed with the subspace and quotient bornology, respectively.

The inclusion $i$ : CBorn $\rightarrow$ Born has a left adjoint, the completion functor Born $\rightarrow$ CBorn, $V \mapsto V^{c}$. This means that there are natural isomorphisms

$$
\operatorname{Hom}(V, W) \cong \operatorname{Hom}\left(V^{c}, W\right)
$$

for $W$ complete. In particular, limits in CBorn are calculated as limits in Born. This is also true for direct sums. However, completion does not preserve colimits in general: the cokernel of a map $f: V \rightarrow W$ in CBorn is obtained by modding out the bornological closure of the image, Coker $f=W / \overline{f(V)}$ [8, Section 1.3.3]. We may write sep colim, sep Coker, etc. to emphasize that a construction is taken inside CBorn.

\footnotetext{
${ }^{1}$ Meyer [8] denotes Born by $\mathfrak{B o r n}{ }^{1 / 2}$, reserving the notation $\mathfrak{B o r n}$ for the full subcategory of separated bornological spaces.
} 
The space $\operatorname{Hom}(V, W)$ of all bounded linear maps has a natural bornology generated by the uniformly bounded subsets, i.e., those $L \subset \operatorname{Hom}(V, W)$ such that

$$
L(B)=\{f(x) \mid f \in L, x \in B\} \subset W
$$

is bounded for all bounded $B \subset V$. This is complete if $W$ is.

We equip the algebraic tensor product $V_{1} \otimes V_{2}$ with the bornology generated by subsets of the form $B_{1} \otimes B_{2}$, with $B_{1} \subset V_{1}, B_{2} \subset V_{2}$ bounded. We define the completed bornological tensor product by

$$
V_{1} \hat{\otimes} V_{2}=\left(V_{1} \otimes V_{2}\right)^{c} \text {. }
$$

Theorem A.4 ([8, Proposition 1.111]). With the completed tensor product and the bornology on hom-sets defined above, CBorn becomes a closed symmetric monoidal category. This means that $\hat{\otimes}$ is unital, commutative and associative in the appropriate sense, and there are natural isomorphisms

$$
\operatorname{Hom}\left(V_{1} \hat{\otimes} V_{2}, W\right) \cong \operatorname{Hom}\left(V_{1}, \operatorname{Hom}\left(V_{2}, W\right)\right)
$$

for all $V_{1}, V_{2}, W \in$ CBorn. Likewise, Born is closed symmetric monoidal with its uncompleted tensor product $\otimes$.

For $V \in$ Born, denote by $\mathfrak{S}_{d}(V)$ the set of bounded disks of $V$, with the partial order relation of being absorbed: $B_{1} \leq B_{2}$ if $B_{1} \subset c B_{2}$ for some constant $c>0$. This implies that we have an injective, continuous linear map $V_{B_{1}} \rightarrow V_{B_{2}}$ of seminormed spaces. Denote by $\mathfrak{S}_{c}(V)$ the subset of complete bounded disks. By definition, $\mathfrak{S}_{c}(V)$ is cofinal in $\mathfrak{S}_{d}(V)$ if and only if $V$ is complete.

Proposition A.5. Let $V$ be a complete bornological vector space. Then there is a canonical isomorphism

$$
V \cong \operatorname{colim}_{B \in \mathfrak{S}_{c}(V)} V_{B}
$$

in CBorn. If $V \in$ Born, replacing $\mathfrak{S}_{c}(V)$ with $\mathfrak{S}_{d}(V)$ gives a similar isomorphism in Born.

Remark A.6. Complete bornological vector spaces are directly related to the category Ind(Ban) of inductive systems of Banach spaces. The assignment of the directed system $\left\{V_{B}\right\}_{B \in \mathfrak{S}_{c}(V)}$ to $V \in$ CBorn defines the dissection functor diss: Born $\rightarrow \operatorname{Ind}(\operatorname{Ban})$. There are also a versions of dissection for non-complete and non-separated spaces.

In sheaf theory, filtered colimits and their commutation properties with certain kinds of limits play an important role. Thus, we analyze now such colimits. Recall that the underlying set of a filtered colimit of vector spaces (and hence also of bornological vector spaces) is the filtered colimit of the underlying diagram of sets.

Lemma A.7. Let $V=\operatorname{colim}_{i \in I} V_{i}$ be a filtered colimit in Born, and denote by $j_{i}: V_{i} \rightarrow V$ the standard map. Then a subset of $V$ is a bounded disk if and only if it is of the form $j_{i}(B)$ for some $i \in I$ and bounded disk $B \subset V_{i}$.

Proof. Expressing $V$ in terms of coproducts and a coequalizer, we find a map

$$
p: \bigoplus_{i \in I} V_{i} \rightarrow V
$$

such that bounded subsets of $V$ are precisely those of the form $p(B)$ with $B$ bounded. A bounded disk $B$ in the direct sum is given by the convex hull of a finite collection $B_{i_{1}}, \ldots, B_{i_{n}}$ of bounded disks $B_{i_{j}} \subset V_{i_{j}}$. Pick an upper bound $i$ for the collection $\left\{i_{1}, \ldots, i_{n}\right\} \subset I$, and let $B_{i} \subset V_{i}$ be the smallest bounded disk containing the images in $V_{i}$ of each $B_{i_{j}} \subset V_{i_{j}}$. Then clearly $p(B)=p\left(B_{i}\right)$, which finishes the proof. 
Proposition A.8. Small filtered colimits commute with finite limits in Born.

Proof. Let $I$ be a finite indexing category, and $J$ filtered. Fix a diagram $V: I \times J \rightarrow$ Born. We want to show that the natural bounded linear map

$$
\phi: \quad \operatorname{colim} \lim _{i \in I} V(i, j) \rightarrow \lim _{i \in I} \operatorname{colim}_{j \in J} V(i, j)
$$

is an isomorphism. Since filtered colimits of vector spaces commute with finite limits, we know that $\phi$ is a linear isomorphism. It remains to show $\phi$ that has a bounded inverse, that is, every bounded disk $B$ in the codomain is contained in the image of a bounded disk.

By Lemma A.7, each of the bounded disks $B_{i}=p_{i}(B) \subset \operatorname{colim}_{j \in J} V(i, j)$ is the image, through the standard map $\iota_{i, k_{i}}: V\left(i, k_{i}\right) \rightarrow \operatorname{colim}_{j \in J} V(i, j)$, of a bounded disk $B_{i, k_{i}} \subset V\left(i, k_{i}\right)$, for some $k_{i} \in J$. Since $I$ is finite, we can pick an upper bound $k$ for the collection $\left\{k_{i}\right\}_{i \in I}$, and we can write $B_{i}=j_{k}\left(B_{i, k}\right)$ for suitable bounded disks $B_{i, k} \subset V(i, k)$.

Now, $\lim _{i \in I} V(i, k)$ is a subspace of the direct product $\prod_{i \in I} V(i, k)$, which, again by finiteness, is also a direct sum. The bounded disks $B_{i, k} \subset V(i, k)$ span a bounded disk in that direct sum, and by restriction, a bounded disk $B_{k} \subset \lim _{i \in I} V(i, k)$. This in turns determines a bounded disk $B^{\prime}=\iota_{k}\left(B_{k}\right)$ in the domain of $\phi$, and $\phi\left(B^{\prime}\right)=B$.

In general, a filtered colimit of separated, or even complete, bornological spaces need not be separated, as the following example shows.

Example A.9. Let $X$ be a manifold and $x \in X$. Consider the space of germs of smooth functions around $x$,

$$
C_{X, x}^{\infty}=\operatorname{colim}_{U \ni x} C^{\infty}(U)
$$

Endowing $C^{\infty}(U)$ with its usual von Neumann bornology and taking the colimit in Born gives $C_{X, x}^{\infty}$ a bornology. Then the germ of a function $f \in C^{\infty}(X)$ which vanishes to infinite order at 0 is in the closure of $\{0\}$ in $C_{X, x}^{\infty}$. In fact, we can find a sequence of functions $f_{n} \in C^{\infty}(X)$ with trivial germ at 0 converging to $f$ in the Fréchet sense, so (equivalently) in the bornological sense. Therefore, the same holds for their images in $C_{X, x}^{\infty}$.

On the other hand, filtered colimits $V=\operatorname{colim} V_{i}$ of separated or complete bornological spaces remain separated or complete for diagrams with only injective morphisms or, more generally, if every bounded $B_{i} \subset V_{i}$ with $j_{i}\left(B_{i}\right)=0 \subset V$ is already in the kernel of some morphism $V_{i} \rightarrow V_{j}$ of the diagram. Such inductive systems are called stable by Houzel [5].

Proposition A.10. Let $\left\{V_{i}\right\}_{i \in I}$ be a stable filtered diagram of separated or complete bornological spaces. Then $V=\operatorname{colim}_{i \in I} V_{i}$ is separated or complete, respectively.

Proof. Let $B \subset V$ be a bounded disk. Then there exists $i \in I$ and a bounded disk $B_{i} \subset V_{i}$ such that $j_{i}\left(B_{i}\right)=B$. Now, $B_{i} \cap \operatorname{Ker} j_{i}$ is a bounded disk with trivial image in $V$, so it has trivial image already in $V_{k}$ for some $k \in I$. Let $B_{k}$ be the image of $B_{i}$ in $V_{k}$, so that $B=j_{k}\left(B_{k}\right)$. Then $\left(V_{k}\right)_{B_{k}} \rightarrow V_{B}$ is injective, so $V_{B}$ is a normed space.

Proposition A.11. Let $V, W \in$ CBorn and assume $V$ has the fine bornology. Then $V \otimes W$ is already complete. If both $V$ and $W$ have the fine bornology, the same is true of their tensor product.

Proof. The first assertion is clear if $V$ is finite-dimensional. Otherwise, we have, by Proposition A.5, $V \cong$ colim $V_{\alpha}$, where $V_{\alpha}$ ranges through the collection of finite-dimensional subspaces 
of $V$, endowed with their fine bornologies and partially ordered by inclusion. Since, like any left adjoint, tensor product commutes with colimits, we have

$$
V \otimes W \cong \operatorname{colim}\left(V_{\alpha} \otimes W\right) .
$$

This is a stable filtered colimit of complete bornological spaces, hence complete. If also $W$ is fine, then

$$
V \otimes W \cong \operatorname{colim}\left(V_{\alpha} \otimes W_{\beta}\right)
$$

where $V_{\alpha} \subset V$ and $W_{\beta} \subset W$ range through all finite-dimensional subspaces. This is a cofinal subcollection of the finite-dimensional subspaces of $V \otimes W$, and therefore induces the fine bornology.

\section{A.2 Bornological algebras and modules}

It is straightforward to define the notion of bounded bilinear maps $V_{1} \times V_{2} \rightarrow W$ between complete bornological vector spaces, and a bornology on the space $\operatorname{Hom}^{(2)}\left(V_{1}, V_{2} ; W\right)$ of such. Moreover, there is a natural isomorphism

$$
\operatorname{Hom}^{(2)}\left(V_{1}, V_{2} ; W\right) \cong \operatorname{Hom}\left(V_{1} \hat{\otimes} V_{2}, W\right) .
$$

A bornological algebra is a bornological vector space $A$ with a bounded, associative bilinear product $A \times A \rightarrow A$. A bornological module is a bornological vector space $M$ with a bounded, associative, bilinear action map $A \times M \rightarrow M$. Any of those is called complete if the underlying bornology is complete.

In this paper we only consider unital, commutative bornological algebras and unital modules, so we drop the extra adjectives. Moreover, we focus on the complete case. Thus we get a category CAlg of complete bornological algebras and, for each $A \in \mathrm{CAlg}$, a category $\operatorname{CMod}(A)$ of complete bornological modules. Module categories are enriched in themselves, that is, for $M, N \in \operatorname{CMod}(A)$, the space of $A$-linear maps $\operatorname{Hom}_{A}(M, N)$, with its subspace bornology, is naturally a complete $A$-module.

By Proposition A.11, the functor Fin: Vect $\rightarrow$ CBorn sends algebras and modules over them to complete bornological algebras and modules. Similarly, a Fréchet algebra $A$ induces a complete bornological algebra structure on vN $(A)$, and a Fréchet $A$-module $M$ gives a complete $\mathrm{vN}(A)$-module structure on $\mathrm{vN}(M)$ [8, Theorem 1.29].

Given modules $M$ and $N$ over the bornological algebra $A$, we set

$$
M \hat{\otimes}_{A} N=\operatorname{Coker}(M \hat{\otimes} A \hat{\otimes} N \rightarrow M \hat{\otimes} N),
$$

where the map is specified by $(m, a, n) \mapsto a m \otimes n-m \otimes a n$.

The usual adjunction between extension and restriction of scalars carries over to the bornological setting. Below, we use $j$ to regard $B$ and $N$ as $A$-modules.

Proposition A.12. Let $j: A \rightarrow B$ be a map of complete bornological algebras and let $M, N$ be complete modules over $A$ and $B$ respectively. Then there are natural isomorphisms

$$
\operatorname{Hom}_{B}\left(B \hat{\otimes}_{A} M, N\right) \cong \operatorname{Hom}_{A}(M, N) .
$$

Moreover, the extension of scalars functor $B \hat{\otimes}_{A}-: \operatorname{CMod}(A) \rightarrow \operatorname{CMod}(B)$ is monoidal.

Proof. Since limits in CBorn commute with the forgetful functor to Vect, we can identify the left-hand side with those bounded bilinear maps $f: B \times M \rightarrow N$ such that

$$
f\left(b b^{\prime}, m\right)=b f\left(b^{\prime}, m\right), \quad f(b j(a), m)=f(b, a m)
$$


for all $a \in A, b, b^{\prime} \in B$ and $m \in M$. With this observation, the usual algebraic manipulations give a linear bijection between the hom spaces in question, and it is easy to check that everything is compatible with the bornologies.

Letting $j=\mathrm{id}: A \rightarrow A$, we see that $A \hat{\otimes}_{A} M \cong M$. From this, and the fact that tensor products over $A$ and $B$ are associative, one deduces that $B \hat{\otimes}_{A}$ - is monoidal.

\section{A.3 Smooth functions}

Given a smooth manifold $S$, we denote by $C_{\mathrm{vN}}^{\infty}(S)$ the space of smooth functions with the von Neumann bornology associated to its usual Fréchet topology. This can be described as the bornology of uniform boundedness of all derivatives of each given order on compacts. If $V$ is a complete bornological space and $f: S \rightarrow V$ a function, we say that $f$ is smooth if there exists a complete bounded disk $B \subset V$ such that $f$ takes values in $V_{B}$, and $f: S \rightarrow V_{B}$ is smooth as a function with values in a Banach space.

Proposition A.13. For any smooth manifold $S$ and complete bornological vector space $V$, there is a natural isomorphism of vector spaces

$$
C^{\infty}(S ; V) \cong C_{\mathrm{vN}}^{\infty}(S) \hat{\otimes} V \text {. }
$$

This defines a bornology on the left-hand side, which we denote $C_{\mathrm{vN}}^{\infty}(S ; V)$.

Proof. Using the dissection isomorphism $V=\operatorname{colim}_{B} V_{B}$, where $B$ ranges over all complete bounded disks in $V$ (Proposition A.5), we get

$$
C^{\infty}(S) \hat{\otimes} V \cong \operatorname{colim}_{B} C^{\infty}(S) \hat{\otimes} V_{B}
$$

On the other hand, we have $C^{\infty}(S ; V)=\operatorname{colim}_{B} C^{\infty}\left(S, V_{B}\right)$ by definition. This reduces the proposition to the case $V=\mathrm{vN}\left(V_{\nu}\right)$ for a Banach space $V_{\nu}$.

The rest of the argument is functional analysis. We have $C^{\infty}\left(S ; \operatorname{vN}\left(V_{\nu}\right)\right)=\operatorname{vN}\left(C^{\infty}\left(S ; V_{\nu}\right)\right)$ by [7, Corollary 3.9]. It is well known [14, Theorem 44.1] that $C^{\infty}\left(S ; V_{\nu}\right) \cong C^{\infty}(S) \hat{\otimes}_{\pi} V_{\nu}$, where we used the completed projective tensor product. Finally, $\mathrm{vN}(V) \hat{\otimes} \mathrm{vN}(W) \cong \mathrm{vN}\left(V \hat{\otimes}_{\pi} W\right)$ if $V$ is nuclear [8, Theorem 1.91], which finishes the proof, since $C^{\infty}(S)$ is nuclear for any manifold $S$ (see, e.g., the corollary of Theorem 51.5 in [14]).

Proposition A.14. For any smooth manifolds $S$ and $T$, we have

$$
C_{\mathrm{vN}}^{\infty}(S \times T) \cong C_{\mathrm{vN}}^{\infty}(S) \hat{\otimes} C_{\mathrm{vN}}^{\infty}(T)
$$

Proof. This follows from the corresponding statement in the Fréchet setting, using the completed projective tensor product [14, Theorem 51.6], and the fact that $\mathrm{vN}(V) \hat{\otimes} \mathrm{vN}(W) \cong$ $\mathrm{vN}\left(V \hat{\otimes}_{\pi} W\right)$ if $V$ is nuclear.

\section{A.4 Bornological sheaves}

In this section, we outline the theory of sheaves of bornological vector spaces. We follow Houzel [5, Section 2].

Let $\mathcal{C}$ be a category with limits, and denote by $\operatorname{PSh}_{\mathcal{C}}(X)$ and $\operatorname{Sh}_{\mathcal{C}}(X)$ the categories of presheaves and sheaves with values in $\mathcal{C}$ on a topological space (or, more generally, a Grothendieck site) $X$. We are interested in the cases $\mathcal{C}=$ CBorn and, as a preliminary step, $\mathcal{C}=$ Born. We will say that a (pre)sheaf with values in Born is complete if it takes values in CBorn. 
The first item in our wish list is to have a left adjoint to the inclusion $\operatorname{Sh}_{\mathcal{C}}(X) \rightarrow \operatorname{PSh}_{\mathcal{C}}(X)$, which we call the sheafification functor. Let $F \in \operatorname{PSh}_{\mathcal{C}}(X), U \subset X$ open, and $\mathfrak{U}=\left\{U_{i} \subset U\right\}_{i \in I}$ be an open cover. As usual, we write

$$
F(\mathfrak{U})=\lim \left(\prod_{i \in I} F\left(U_{i}\right) \rightrightarrows \prod_{i, j \in I} F\left(U_{i} \cap U_{j}\right)\right)
$$

and say that $F$ is a sheaf if the canonical map $F(U) \rightarrow F(\mathfrak{U})$ an isomorphism for all $\mathfrak{U}$. We will call $F$ a semisheaf if the maps $F(U) \rightarrow F(\mathfrak{U})$ are always monomorphisms. Since the inclusion CBorn $\subset$ Born preserves limits, the condition of being a (semi)sheaf in one of these categories is equivalent to being a (semi)sheaf in the other. Set

$$
F^{+}(U)=\operatorname{colim}_{\mathfrak{U}} F(\mathfrak{U}),
$$

where $\mathfrak{U}$ runs through all open covers of $X$. For the traditional choices $\mathcal{C}=$ Set, Vect, etc., $F^{+}$ is a semisheaf, and is a sheaf if $F$ is already a semisheaf. This defines the sheafification functor $F \mapsto F^{++}$. When $\mathcal{C}=$ Born, $F^{++}$is obviously a sheaf when regarded as taking values in Vect, and it can be checked directly that it is in fact a bornological sheaf.

If $F \in \mathrm{PSh}_{\text {Born }}(X)$ is a complete semisheaf, then the inductive system defining $F^{+}$is stable, so it follows, from Proposition A.10, that $F^{+}$is a complete sheaf. However, if $F$ fails to be a semisheaf, then $F^{+}$may not be complete even if $F$ is. This issue is resolved in Houzel [5, p. 32] as follows. Let $F \in \mathrm{PSh}_{\text {Born }}$ be arbitrary and let $E$ be the smallest sub-presheaf of $U \mapsto \widehat{F(U)}$ such that $F^{\prime}: U \mapsto \widehat{F(U)} / E(U)$ is a complete semisheaf. Then $\left(F^{\prime}\right)^{+}$is a complete sheaf, and this construction gives a left adjoint to the inclusion $\operatorname{Sh}_{\mathrm{CBorn}}(X) \rightarrow \mathrm{PSh}_{\mathrm{Born}}(X)$. By restriction, this left adjoint gives us both a sheafification functor $\mathrm{PSh}_{\mathrm{CBorn}}(X) \rightarrow \mathrm{Sh}_{\mathrm{CBorn}}$ and a completion functor $\mathrm{Sh}_{\mathrm{Born}} \rightarrow \mathrm{Sh}_{\mathrm{CBorn}}$.

The second item in our wish list is to get tensor products and internal homs. If $E, F \in$ $\operatorname{PSh}_{\text {Born }}(X)$, then $\operatorname{Hom}(E, F)$ gets a bornology as a subspace of the product $\prod_{U \subset X} \operatorname{Hom}(E(U)$, $F(U)), U \subset X$ open, and we get also a Born-valued presheaf $\underline{\operatorname{Hom}}(E, F)$. It is a sheaf if so is $F$, and it is complete if so is $F$. We define $E \otimes F$ as the sheafification of the presheaf $U \mapsto E(U) \otimes F(U)$, and $E \hat{\otimes} F$ as the associated complete sheaf.

The following statement summarizes this discussion.

Theorem A.15. Let $\mathcal{C}=$ Born or CBorn and let $X$ be a topological space. Then the inclusion $\operatorname{Sh}_{\mathcal{C}}(X) \rightarrow \operatorname{PSh}_{\mathcal{C}}(X)$ admits a left adjoint. Moreover, with the hom-sheaf and tensor product defined above, $\mathrm{Sh}_{\mathcal{C}}(X)$ is a closed symmetric monoidal category.

If $f: X \rightarrow Y$ is a continuous map and $E \in \operatorname{PSh}_{\text {Born }}(X)$, then the direct image $f_{*} E$ is the presheaf $U \mapsto E\left(f^{-1}(U)\right), U \subset Y$ open. It is a sheaf if $E$ is. For $F \in \operatorname{Sh}_{\text {Born }}(Y)$, let $f^{-1}(F)$ is the sheafification of the presheaf $U \mapsto \operatorname{colim}_{V \supset f(U)} F(V)$. This defines a left adjoint

$$
f^{-1}: \operatorname{Sh}_{\text {Born }}(Y) \rightarrow \operatorname{Sh}_{\text {Born }}(X) .
$$

to $f_{*}$. If $g: Y \rightarrow Z$ is another map, then $g_{*} \circ f_{*} \cong(g \circ f)_{*}$ and $f^{-1} \circ g^{-1} \cong(g \circ f)^{-1}$. The first fact is obvious and the second follows because $f^{-1}$ is adjoint to $f_{*}$.

Note also that if $f$ is an open map and $F$ takes values in CBorn, then so does $f^{-1}(F)$.

Finally, we turn to sheaves of algebras and modules. Let $A$ be a sheaf of complete bornological algebras over $X$, and denote by $\operatorname{CMod}(A)$ the category of sheaves of complete $A$-modules. If $M, M^{\prime} \in \operatorname{CMod}(A)$, we denote by $\underline{\operatorname{Hom}}_{A}\left(M, M^{\prime}\right)$ the subsheaf of $\underline{\operatorname{Hom}}\left(M, M^{\prime}\right)$ consisting of $A$-linear morphisms. It is complete if $M^{\prime}$ is. The tensor product $M \hat{\otimes}_{A} M^{\prime}$ is the complete sheaf associated to the presheaf $U \mapsto M(U) \otimes_{A(U)} M^{\prime}(U)$. Assume now we are given a map $f: X \rightarrow Y$. Then $f_{*} A$ is naturally a sheaf of bornological algebras, and $f_{*} M$ an $f_{*} A$-module. Also, if $B$ is a sheaf of bornological algebras on $Y$ and $N \in \operatorname{CMod}(B)$ is a module, then $f^{-1}(B)$ is naturally a sheaf of bornological algebras, and $f^{-1}(N)$ an $f^{-1}(B)$-module. 


\section{A.5 The stack $\mathcal{V}_{\mathrm{vN}}$}

The assignment $U \mapsto C_{\mathrm{vN}}^{\infty}(U)$ defines a sheaf of complete bornological algebras on the site Man of smooth manifolds. Its restriction to the small site of a manifold $S$ will be denoted $C_{\mathrm{vN}, S}^{\infty}$.

We denote by $\mathcal{V}_{\mathrm{vN}}(S)=\operatorname{CMod}\left(C_{\mathrm{vN}, S}^{\infty}\right)$ the category of sheaves of complete $C_{\mathrm{vN}, S}^{\infty}$-modules. As observed in the previous subsection, this is a closed symmetric monoidal category.

Any smooth map $f: T \rightarrow S$ induces a homomorphism $C_{\mathrm{vN}, S}^{\infty} \rightarrow f_{*} C_{\mathrm{vN}, T}^{\infty}$ of sheaves of complete bornological algebras. We denote by $f^{\sharp}: f^{-1}\left(C_{\mathrm{vN}, S}^{\infty}\right) \rightarrow C_{\mathrm{vN}, T}^{\infty}$ its adjoint. This makes $C_{\mathrm{vN}, T}^{\infty}$ into a sheaf of $f^{-1}\left(C_{\mathrm{vN}, S}^{\infty}\right)$-modules. Given $M \in \mathcal{V}_{\mathrm{vN}}(S)$, we set

$$
\hat{f}^{*} M=C_{\mathrm{vN}, T}^{\infty} \hat{\otimes}_{f^{-1}\left(C_{\mathrm{vN}, S}^{\infty}\right)} f^{-1}(M) .
$$

This defines a symmetric monoidal functor

$$
\hat{f}^{*}: \mathcal{V}_{\mathrm{vN}}(S) \rightarrow \mathcal{V}_{\mathrm{vN}}(T),
$$

and the assignments $S \rightarrow \mathcal{V}_{\mathrm{vN}}(S)$ and $f \mapsto \hat{f}^{*}$ determine a prestack of symmetric monoidal categories

$$
\mathcal{V}_{\mathrm{vN}}: \mathrm{Man}^{\mathrm{op}} \rightarrow \mathrm{Cat}^{\otimes}
$$

Finally, we need to show that $\mathcal{V}_{\mathrm{vN}}$ is in fact a stack. This is the case because the pretopology of all open covers of a manifold $S$ and the pretopology of open covers subordinated to a given open cover generate the same Grothendieck site. More concretely, if $\mathfrak{U}=\left\{U_{i} \subset S\right\}$ is an open cover and $V_{i} \in \mathcal{V}_{\mathrm{vN}}\left(U_{i}\right)$ is a collection of objects with coherent isomorphisms between their restrictions to overlaps $U_{i} \cap U_{j}$, we can construct a presheaf of $C_{\mathrm{vN}, S}^{\infty}$-modules $V^{\prime}$ which agrees with $V_{i}$ on $U_{i}$ and assigns 0 to any open not contained in some $U_{i}$. Sheafification then gives the desired "glued together" object $V \in \mathcal{V}_{\mathrm{vN}}(S)$.

\section{Acknowledgments}

We thank Dmitri Pavlov, Peter Teichner, Konrad Waldorf and, especially, Stephan Stolz for helpful discussions. We are further indebted to the Max Planck Institute for Mathematics in Bonn and the University of Greifswald, where part of this research was conducted. The firstnamed author was partially supported by the ARC Discovery Project grant FL170100020 under Chief Investigator and Australian Laureate Fellow Mathai Varghese.

\section{References}

[1] Atiyah M., Topological quantum field theories, Inst. Hautes Études Sci. Publ. Math. 68 (1988), $175-186$.

[2] Berwick-Evans D., Pavlov D., Smooth one-dimensional topological field theories are vector bundles with connection, arXiv:1501.00967.

[3] Bunke U., Turner P., Willerton S., Gerbes and homotopy quantum field theories, Algebr. Geom. Topol. 4 (2004), 407-437, arXiv:math.AT/0201116.

[4] Freed D.S., Classical Chern-Simons theory. I, Adv. Math. 113 (1995), 237-303, arXiv:hep-th/9206021.

[5] Houzel C., Espaces analytiques relatifs et théorème de finitude, Math. Ann. 205 (1973), 13-54.

[6] Metzler D., Topological and smooth stacks, arXiv:math.DG/0306176.

[7] Meyer R., Bornological versus topological analysis in metrizable spaces, in Banach Algebras and their Applications, Contemp. Math., Vol. 363, Amer. Math. Soc., Providence, RI, 2004, 249-278, arXiv:math.FA/0310225.

[8] Meyer R., Local and analytic cyclic homology, EMS Tracts in Mathematics, Vol. 3, European Mathematical Society (EMS), Zürich, 2007. 
[9] Rezk C., A model for the homotopy theory of homotopy theory, Trans. Amer. Math. Soc. 353 (2001), 973-1007, arXiv:math.AT/9811037.

[10] Schreiber U., Waldorf K., Parallel transport and functors, J. Homotopy Relat. Struct. 4 (2009), 187-244, arXiv:0705.0452.

[11] Segal G., Classifying spaces and spectral sequences, Inst. Hautes Études Sci. Publ. Math. 34 (1968), $105-112$.

[12] Segal G., The definition of conformal field theory, in Topology, Geometry and Quantum Field Theory, London Math. Soc. Lecture Note Ser., Vol. 308, Cambridge University Press, Cambridge, 2004, 421-577.

[13] Stolz S., Teichner P., Supersymmetric field theories and generalized cohomology, in Mathematical Foundations of Quantum Field Theory and Perturbative String Theory, Proc. Sympos. Pure Math., Vol. 83, Amer. Math. Soc., Providence, RI, 2011, 279-340, arXiv:1108.0189.

[14] Trèves F., Topological vector spaces, distributions and kernels, Academic Press, New York - London, 1967.

[15] Vistoli A., Grothendieck topologies, fibered categories and descent theory, in Fundamental Algebraic Geometry, Math. Surveys Monogr., Vol. 123, Amer. Math. Soc., Providence, RI, 2005, 1-104, arXiv:math.AG/0412512.

[16] Witten E., Topological quantum field theory, Comm. Math. Phys. 117 (1988), 353-386. 